\title{
Stability properties of the transverse envelope equations describing intense ion beam transport
}

\author{
Steven M. Lund* \\ Lawrence Livermore National Laboratory, University of California, Livermore, California 94550, USA \\ Boris Bukh \\ Lawrence Berkeley National Laboratory, University of California, Berkeley, California 94720, USA
}

(Received 13 June 2003; published 11 February 2004)

\begin{abstract}
The transverse evolution of the envelope of an intense, unbunched ion beam in a linear transport channel can be modeled for the approximation of linear self-fields by the Kapchinskij-Vladimirskij (KV) envelope equations. Here we employ the KV envelope equations to analyze the linear stability properties of so-called mismatch perturbations about the matched (i.e., periodic) beam envelope in continuous focusing, periodic solenoidal, and periodic quadrupole transport lattices for a coasting beam. The formulation is analyzed and explicit self-consistent $\mathrm{KV}$ distributions are derived for an elliptical beam envelope in a periodic solenoidal transport channel. This derivation extends previous work to identify emittance measures and Larmor-frame transformations to allow application of standard form envelope equations to solenoidal focusing channels. Perturbed envelope equations are derived that include driving sources of mismatch excitation resulting from focusing errors, particle loss, and beam emittance growth. These equations are solved analytically for continuous focusing and demonstrate a factor of 2 increase in maximum mismatch excursions resulting from sudden driving perturbations relative to adiabatic driving perturbations. Numerical and analytical studies are carried out to explore properties of normal mode envelope oscillations without driving excitations in periodic solenoidal and quadrupole focusing lattices. Previous work on this topic by Struckmeier and Reiser [Part. Accel. 14, 227 (1984)] is extended and clarified. Regions of parametric instability are mapped, new classes of envelope instabilities are found, parametric sensitivities are explored, general limits and mode invariants are derived, and analytically accessible limits are checked. Important, and previously unexplored, launching conditions are described for pure envelope modes in periodic quadrupole focusing channels.
\end{abstract}

DOI: 10.1103/PhysRevSTAB.7.024801

PACS numbers: 29.27.Bd, 41.75.-i, 52.59.Sa, 52.27.Jt

\section{INTRODUCTION}

Intense ion beams are usually transported in a periodic lattice of linear focusing elements that radially confine the beam against defocusing space-charge and thermal forces arising from the distribution of beam particles. Envelope equations are frequently used to model the low-order, statistical evolution of the beam edge. The Kapchinskij-Vladimirskij (KV) envelope equations are coupled ordinary differential equations that describe the transverse evolution of the edge of an unbunched elliptical beam in a linear focusing lattice consistent with linear space-charge defocusing forces and constant emittances (i.e, invariant phase-space projections) [1-3]. The $\mathrm{KV}$ envelope equations are consistent with the KV distribution which is an equilibrium solution of the Vlasov equation. The KV distribution corresponds to a uniformly populated three-dimensional hyperellipsoid in fourdimensional transverse phase space. It is self-consistent because it is constructed from invariants of single particles moving in linear applied and space-charge fields and yields (consistent) distribution projections that produce linear self-fields. Even though the KV distribution is

*Electronic address: smlund@lbl.gov unphysical due to its singular hypershell structure which provides a source of free energy to drive higher-order collective instabilities internal to the beam [4], the KV envelope equations are valid for general distributions with evolving emittances in a rms equivalent beam sense $[2,5,6]$. Moreover, because space-charge profiles on intense beams tend to be fairly uniform in the core of the beam, the linear self-fields in the KV model should be a good approximation at high space-charge intensities except in a thin layer near the beam edge where the charge density drops rapidly to zero. Consequently, for intense beams propagating in linear transport channels without significant particle loss or emittance growth, the KV envelope equations can provide a good estimate of the actual (rms statistical measure) envelope of the beam. Because of this and the fact that the ordinary differential equations describing the $\mathrm{KV}$ envelope evolution are straightforward to solve numerically, the KV envelope equations are extensively employed in the design of practical linear transport lattices.

The matched beam envelope is the solution to the KV envelope equations with the periodicity of the focusing lattice. The matched solution is generally believed to have the smallest maximum radial excursions relative to other possible envelope evolutions in the lattice and it requires particular initial conditions in the envelope of beam 
particles. There will always be some finite mismatch error or deviation of the beam envelope from this matched evolution. These mismatch errors can be analyzed in terms of the small-amplitude modes about the matched solution that are supported by the KV envelope equations $[2,7]$. Parametric instabilities of the mismatch modes must be avoided for practical machine operating points to maintain beam control. Even in cases where the envelope is stable, the structure of the mismatch modes is important. The frequencies of the envelope modes can resonate with lattice structures, particle orbits, and collective space-charge waves causing deleterious beam effects. For example, the high frequency breathing envelope mode is well known to drive large-amplitude resonant beam halo causing loss of beam particles [8]. Therefore the control of this mode, even when stable, can be of critical importance.

In this paper we carry out a systematic parametric analysis of mismatch modes supported by the KV envelope equations for continuous focusing, periodic solenoid, and periodic quadrupole doublet linear transport lattices. Regions of strong parametric (band) instability for the periodic lattices are numerically mapped in a scaled manner rendering results immediately applicable to a large range of beam parameters and lattices. New classes of envelope instability are found and results are checked against analytical calculations of the envelope modes in the zero space-charge limit and in the thin-lens limit with a beam of maximal space-charge intensity. Important and often overlooked mode launching conditions and sources of envelope mismatch (driving terms) due to focusing errors, particle loss, and emittance evolution are analyzed. These launching conditions are of practical importance in understanding how to launch pure mode oscillations in experiments and simulations which can aid in understanding complex effects.

This paper is organized as follows. In Sec. II, the envelope model employed is developed. The basic equations are reviewed, classes of transport lattices are defined, characteristic envelope responses are calculated, convenient parametrizations are identified, equations describing small-amplitude driven perturbations about the matched beam envelope are derived, and general properties of envelope mode solutions are analyzed. Selfconsistent $\mathrm{KV}$ distributions for solenoidal focusing channels are developed in Appendix A. These results are important to establish the validity of models employed. In Sec. III, the linear mode structure is completely solved in the continuous focusing limit employing a Green's function approach and the structures of the formal solutions are illustrated with specific examples to understand the differences between adiabatic, sudden, and harmonic driven perturbations. Next, normal envelope modes of periodic solenoidal and quadrupole doublet lattices are analyzed in Secs. IV and V. In both sections numerical results are presented that illustrate properties of the matched envelope solutions, mode properties and symmetries, regions of parametric (band) instability, and pure mode launching conditions.

\section{MODEL EQUATIONS}

We consider an unbunched beam of ions of charge $q$ and mass $m$ coasting with axial relativistic factors $\beta_{b}=$ const and $\gamma_{b}=1 / \sqrt{1-\beta_{b}^{2}}$. The ions are confined transversely by linear applied focusing fields that may result from a variety of focusing systems. Linear space-charge forces internal to the beam envelope are assumed, corresponding to a spatially uniform distribution of charge within an elliptical beam envelope with beam image charges induced on any aperture structures neglected.

\section{A. KV envelope equations}

As illustrated in Fig. 1, the transverse beam cross section at axial coordinate $s$ is taken to have an $s$-varying elliptical cross section with envelope (i.e., edge) radii $r_{x}(s)$ and $r_{y}(s)$ along the principal $x$ - and $y$-coordinate axes. The charge distribution is centered at $x=y=0$ with uniform space charge within the ellipse $\left(x / r_{x}\right)^{2}+\left(y / r_{y}\right)^{2}=1$, and zero outside. The $x$ and $y$ equations of motion for the orbit of a single particle within this uniform density elliptical beam are given by $[1,2]$

$$
\begin{aligned}
& x^{\prime \prime}(s)+\kappa_{x}(s) x(s)-\frac{2 Q x(s)}{\left[r_{x}(s)+r_{y}(s)\right] r_{x}(s)}=0, \\
& y^{\prime \prime}(s)+\kappa_{y}(s) y(s)-\frac{2 Q y(s)}{\left[r_{x}(s)+r_{y}(s)\right] r_{y}(s)}=0,
\end{aligned}
$$

where $\kappa_{x}(s)$ and $\kappa_{y}(s)$ represent linear applied forces of focusing optics in the transport lattice, and

$$
Q=\frac{q \lambda}{2 \pi \epsilon_{0} m c^{2} \gamma_{b}^{3} \beta_{b}^{2}}=\text { const }
$$

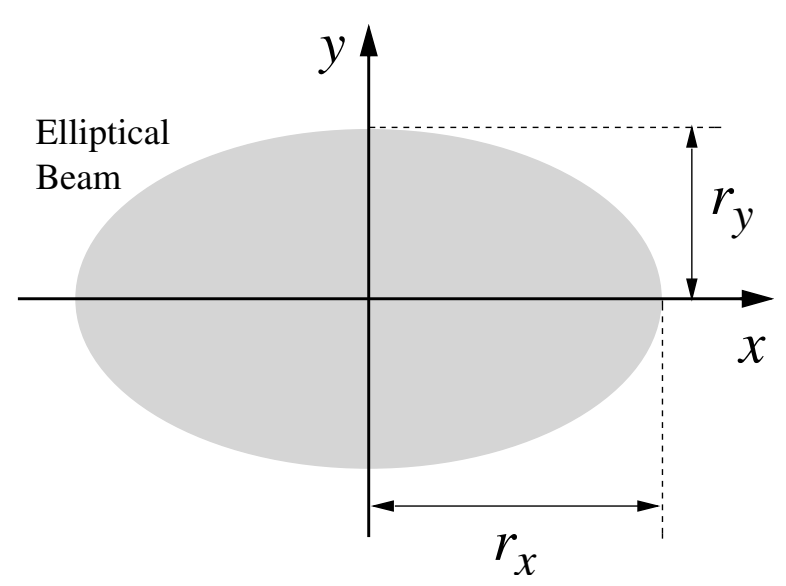

FIG. 1. Transverse cross section of an elliptical beam centered at $x=y=0$ with envelope radii $r_{x}$ and $r_{y}$ along the $x$ and $y$ axes and uniform charge density within the ellipse. 
is the dimensionless beam perveance representing selffield defocusing forces internal to the beam. Here, $\lambda=$ const is the beam line-charge density, $c$ is the speed of light in vасиио, and $\epsilon_{0}$ is the permittivity of free space. In the limit of negligible beam space charge, $Q \rightarrow 0$ in Eq. (1), and the particle moves solely in response to the applied focusing forces of the lattice.

For a uniform density elliptical beam, the envelope radii $r_{x}$ and $r_{y}$ are connected to statistical moments of the distribution by

$$
r_{x}=2 \sqrt{\left\langle x^{2}\right\rangle_{\perp}}, \quad r_{y}=2 \sqrt{\left\langle y^{2}\right\rangle_{\perp}}
$$

where $\langle\cdots\rangle_{\perp}$ denotes a transverse statistical average over the beam phase space. Differentiating Eq. (3) with respect to $s$ and employing the equations of motion (1) yields coupled ordinary differential equations describing the evolution of the envelope radii $r_{x}(s)$ and $r_{y}(s)[1,2,7]$

$$
r_{j}^{\prime \prime}(s)+\kappa_{j}(s) r_{j}(s)-\frac{2 Q}{r_{x}(s)+r_{y}(s)}-\frac{\varepsilon_{j}^{2}}{r_{j}^{3}(s)}=0 .
$$

Here and henceforth $j$ ranges over both transverse coordinates $x$ and $y$, and

$$
\begin{aligned}
& \varepsilon_{x}=4\left[\left\langle x^{2}\right\rangle_{\perp}\left\langle x^{\prime 2}\right\rangle_{\perp}-\left\langle x x^{\prime}\right\rangle_{\perp}^{2}\right]^{1 / 2}, \\
& \varepsilon_{y}=4\left[\left\langle y^{2}\right\rangle_{\perp}\left\langle y^{\prime 2}\right\rangle_{\perp}-\left\langle y y^{\prime}\right\rangle_{\perp}^{2}\right]^{1 / 2}
\end{aligned}
$$

are the rms edge emittances which provide statistical measures of the beam phase-space area projections in the $x-x^{\prime}$ and $y-y^{\prime}$ phase spaces.

For the special case of a KV distribution, the Vlasov single-particle distribution function of the beam is a delta-function form defining a hyper-shell in transverse phase space that yields a uniform-density elliptical beam projection consistent with the particle equations of motion (1) (see Appendix A and Refs. [1-3]). The KV distribution has uniformly filled elliptical projections in the $x-y, x-x^{\prime}, y-y^{\prime}$, and $x-x^{\prime}$ transverse phase spaces and is a Vlasov equilibrium because it is constructed of singleparticle invariants of Eq. (1). For a KV beam, Eq. (4) is fully self-consistent and is referred to as the KVenvelope equation and the statistical emittances (5) are constants of the motion $\left(\varepsilon_{j}=\right.$ const $)$ which are proportional to the phase-space areas in $x-x^{\prime}$ and $y-y^{\prime}$ (i.e., $\pi \varepsilon_{x}=$ const [9] is the area of uniformly filled elliptical distribution projection in $x-x^{\prime}$ ). The singular structure of the KV distribution provides a source of free energy that gives rise to numerous higher-order (Vlasov model) collective instabilities internal to the beam $[4,10]$. However, the loworder projections of the distribution reflected by the envelope equation (4) are reasonable and the KV envelope equations can be reliably applied in transport modeling.

The statistical beam edge radii (3) and emittances (5) are often employed to analyze nonuniform beam distributions not of KV form, extending the range of applicability of the envelope equation (4). In this context, the beam can be interpreted in an rms equivalent beam sense $[2,5,6]$ in terms of an equivalent $\mathrm{KV}$ beam. Two beams are said to be rms equivalent if they have identical particle species, kinetic energy, $Q$, and second-order moments $\left(\left\langle x^{2}\right\rangle_{\perp},\left\langle x^{\prime 2}\right\rangle_{\perp},\left\langle x x^{\prime}\right\rangle_{\perp}\right.$, etc.). In this sense, the statistical rms emittances (5) can be interpreted for a nonuniform density beam in terms of the phase-space areas of a KV equivalent beam. However, for beams with nonuniform charge density the statistical emittances will evolve due to nonlinear self-field forces acting on the particles [2,11]. Work by Sacherer [6] further improves the utility of the equivalent beam concept by showing that the envelope equation (4) is also valid for any two-dimensional charge distributions in free space with elliptical symmetry in $x-y$ (i.e., particle number density $n$ as a function of the radial coordinate $\rho \equiv \sqrt{x^{2} / r_{x}^{2}+y^{2} / r_{y}^{2}}$ ). If a self-consistent Vlasov distribution exists for this evolution [12], the envelope radii $r_{j}$ and emittances $\varepsilon_{j}$ evolve from initial values and density function $n(\rho)$ can also change in form as the beam evolves while elliptical charge symmetry is maintained. In this generalized context the envelope equation (4) is valid if consistent emittance evolutions are employed in the integration. For reasonably smooth initial beam distributions and a transport channel tuned for applied focusing linearity and lack of instabilities, emittance evolutions are often adiabatic $[2,11]$. Moreover, at high space-charge intensities, charge distributions tend to become uniform within the core of the beam out to near a sharp beam edge and the free energy to drive emittance growth becomes small $[13,14]$. Taking these features into account, it is not surprising that the KV envelope equations have much wider practical utility than one might expect from the idealized structure of the KV distribution.

For a particular focusing lattice [i.e., specified focusing functions $\left.\kappa_{j}(s)\right]$ and beam parameters $\left(Q\right.$ and $\left.\varepsilon_{j}\right)$, the $\mathrm{KV}$ envelope equation (4) is typically integrated from an initial condition (i.e., specified $r_{j}$ and $r_{j}^{\prime}$ at $s=s_{i}$ ) to solve for the envelope evolution. In many cases we will take the $x$ and $y$ planes to have equal focusing strength (i.e., $\sigma_{0 x}=$ $\sigma_{0 y} \equiv \sigma_{0}$, see Sec. IID) and constant, equal emittances ( $\varepsilon_{x}=\varepsilon_{y} \equiv \varepsilon=\mathrm{const}$ ) corresponding to a beam with isotropic transverse temperature (on average) and negligible emittance evolution propagating in a linear transport channel without bends, dispersion, and edge focusing.

\section{B. Periodic transport channels and beam matching}

In a periodic transport lattice, applied focusing elements are arranged in a periodic sequence with lattice period $L_{p}$ and lattice focusing functions $\kappa_{j}(s)$ satisfying

$$
\kappa_{j}\left(s+L_{p}\right)=\kappa_{j}(s) .
$$

The matched beam envelope is the solution to the KV envelope equation (4) in a periodic transport where the 
envelope radii $r_{j}(s)$ are also periodic functions with a period of the lattice, i.e., $r_{j}(s)=r_{j m}(s)$ with

$$
r_{j m}\left(s+L_{p}\right)=r_{j m}(s) .
$$

For specified focusing functions $\kappa_{j}(s)$, beam perveance $Q$, and emittances $\varepsilon_{j}$, this is equivalent to requiring that $r_{j}$ and $r_{j}^{\prime}$ satisfy specific initial conditions at $s=s_{i}$ when the envelope equation (4) is integrated as an initial value problem. Required initial conditions will change with the phase of $s_{i}$ within the lattice period. The matched envelope solution is important because it is believed to have minimum radial excursions in the transport channel relative to all other possible envelope evolutions [15], thereby allowing the use of more radially compact transport channels. No known proofs exist to show that the $r_{j}\left(s_{i}\right)$ and $r_{j}^{\prime}\left(s_{i}\right)$ satisfying the matching requirement (7) are unique, although numerical evidence suggests that this is the case for some simple classes of lattices. Matched solutions may not exist for ranges of lattice and beam parameters. Initial envelope conditions in $r_{j}\left(s_{i}\right)$ and $r_{j}^{\prime}\left(s_{i}\right)$ to satisfy the matching condition (7) to adequate precision are generally found using a numerical root finding from guessed starting values together with numerical integration of the envelope equation (4) with specified $\kappa_{j}(s), Q$, and $\varepsilon_{j}$. The numerical implementation of efficient and robust matching methods is discussed in Appendix B.

Although formulations in the following parts of this section apply for arbitrary periodic focusing functions $\kappa_{j}(s)$, in Secs. III, IV, and V we analyze special classes of (a) continuous, (b) periodic solenoidal, and (c) periodic quadrupole doublet focusing lattices described below with $\kappa_{j}(s)$ that are piecewise constant, corresponding to so-called hard-edge or square-edge models. These three choices are representative of broad classes of lattices used in practical applications and can guide the analysis of more complicated lattices. Examples of matched envelope solutions will be presented for each class of lattice in Secs. III, IV, and V. These hard-edge models can be applied to a wide range of periodic solenoid and quadrupole lattices with fringe fields where the $\kappa_{j}(s)$ vary smoothly in $s$ by using equivalent hard-edge replacement prescriptions (see Appendix C).

(a) Continuous focusing, with equal and constant focusing in each plane [see Fig. 2(a)] [2]. In this case,

$$
\kappa_{x}(s)=\kappa_{y}(s)=k_{\beta 0}^{2}=\mathrm{const}>0 .
$$

Continuous focusing is equivalent to a partially neutralizing, noninteracting background of charges and is commonly used to simply estimate the average focusing properties of periodic lattices in rapid design estimates. Here, $k_{\beta 0}$ is the wave number of particle oscillations in the absence of space charge (see Sec. IID). The choice of "lattice period" $L_{p}$ for continuous focusing is arbitrary.

(b) Solenoidal focusing, produced by a magnetic field $\mathbf{B}(s)=-\left[B_{z}^{\prime}(s) / 2\right](x \hat{\mathbf{x}}+y \hat{\mathbf{y}})+B_{z}(s) \hat{\mathbf{z}}$ with period $L_{p}$ [see Fig. 2(b)] [2]. In this case,

$$
\kappa_{x}(s)=\kappa_{y}(s) \equiv \kappa(s)=\left[\frac{q B_{z}(s)}{2 m \gamma_{b} \beta_{b} c}\right]^{2}
$$

Here, the envelope equation (4) must be interpreted in a local rotating "Larmor" frame in the sense shown in Appendix A. In analyses of solenoidal focused systems, we will assume that all calculations are carried out in the Larmor frame unless otherwise noted. We define a hardedge lattice where the solenoids have axial length $\eta L_{p}$ with $\kappa=\hat{\kappa}=$ const $>0$ within the solenoid and zero outside and the solenoids are separated by axial drifts of length $d=(1-\eta) L_{p}$. Here, $\eta \in(0,1]$ is the occupancy factor of the solenoid in the lattice period. In the Larmor frame, solenoidal focusing is equivalent to continuous focusing in the limit $\eta \rightarrow 1$.

(c) Quadrupole doublet focusing in an alternating gradient lattice with period $L_{p}$ [see Fig. 2(c)] [2]. In this case,

$$
\kappa_{x}(s)=-\kappa_{y}(s) \equiv \kappa_{q}(s)
$$

The quadrupole focusing fields are commonly derived either from electrostatic or magnetostatic lenses. In terms of a simple 2D field model, the focusing strength for electric lenses can be expressed as

$$
\kappa_{q}=\frac{1}{[B \rho] \beta_{b} c} \frac{d E_{x}}{d x},
$$

where $[B \rho] \equiv \gamma_{b} \beta_{b} m c / q$ is the particle rigidity and $d E_{x} / d x$ is the (generally $s$-varying) linear quadrupole field gradient of the electric field. Similarly, the focusing strength for magnet lenses can be expressed as

$$
\kappa_{q}=\frac{1}{[B \rho]} \frac{d B_{x}}{d y},
$$

where $d B_{x} / d y$ is the (generally $s$-varying) linear quadrupole field gradient of the magnetic field. We define a hard-edge doublet lattice where the focusing $\left(\kappa_{q}>0\right.$ for focusing in $x$ ) and defocusing $\left(\kappa_{q}<0\right)$ quadrupoles have axial length $\eta L_{p} / 2$ with $\kappa_{q}= \pm \hat{\kappa}_{q}$ with $\hat{\kappa}_{q}=$ const within the quadrupoles and zero outside, and are separated by (generally unequal) axial drifts of length $d_{1}=$ $\alpha(1-\eta) L_{p}$ and $d_{2}=(1-\alpha)(1-\eta) L_{p}$. Here, $\eta \in$ $(0,1]$ is the quadrupole occupancy factor in the lattice period, and $\alpha \in[0,1]$ is a syncopation factor that measures how close the focusing and defocusing quadrupoles are to each other in the lattice period. For the special case of $\alpha=1 / 2$ defining a symmetric FODO lattice, the drifts are equal $\left[d_{1}=d_{2}=(1-\eta) L_{p} / 2\right]$ and the lenses are equally spaced. For $\alpha \neq 1 / 2$, the lattice is called syncopated. Without loss of generality, we restrict the analysis to $0 \leq \alpha \leq 1 / 2$ because values of $\alpha$ with $1 / 2 \leq \alpha \leq 1$ 
a) Continuous

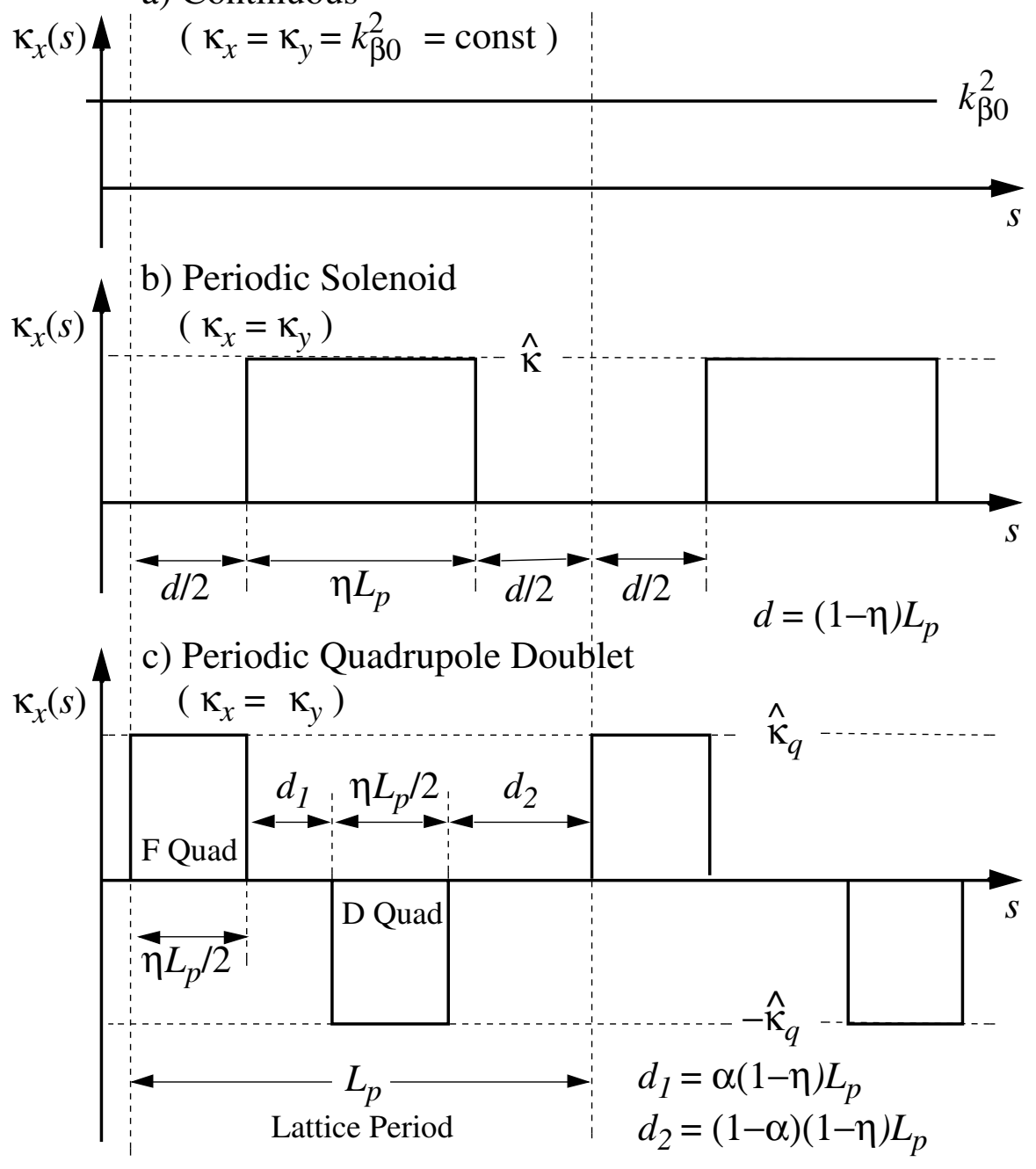

FIG. 2. Linear focusing functions in the $x$ plane for (a) continuous focusing, (b) periodic solenoidal focusing, and (c) periodic quadrupole doublet focusing.

can be mapped to the range $0 \leq \alpha \leq 1 / 2$ by relabeling lattice quantities.

\section{Characteristic envelope responses}

The envelope evolution in the periodic, hard-edge focusing lattices described in Sec. II B can be interpreted in terms of the sequential response of the envelope to the focusing action of optical elements followed by free-drift expansions without focusing $\left(\kappa_{j}=0\right)$.

No general analytical solutions to the envelope evolution within hard-edge solenoidal or quadrupole optics are known for arbitrary values of $Q$ and $\varepsilon_{j}$. Some simplifications can be made using the envelope Hamiltonian as a constant of the motion within a particular optical element, but full integrations of the envelope trajectories $r_{j}(s)$ are not known. However, the essential effect of the optics can be simply understood in the thin-lens limit where $\eta \rightarrow 0$ while adjusting the focusing strength such that the desired net particle focusing (measured by $\sigma_{0 j}$, see Sec. IID) is produced. This is effected by taking

$$
\kappa_{j}(s)=\frac{1}{f} \delta\left(s-s_{o}\right),
$$

where $\delta(s)$ is the Dirac delta function, $f=$ const is the thin-lens focal length, and $s=s_{o}$ is the axial location of the equivalent model optical impulse. In this thin-lens limit, it follows from Eqs. (4) and (13) that the action of the optic is to transform the envelope as

$$
\left(\begin{array}{l}
r_{j} \\
r_{j}^{\prime}
\end{array}\right)_{s_{o}^{+}}=\left.\left[\begin{array}{cc}
1 & 0 \\
-\frac{1}{f} & 1
\end{array}\right]\left(\begin{array}{c}
r_{j} \\
r_{j}^{\prime}
\end{array}\right)\right|_{s_{o}^{-}},
$$

where $\left.\right|_{s_{o}^{ \pm}}$denotes the $\operatorname{limit} \lim _{s \rightarrow s_{o}}$ from above and below $s=s_{o}$. For solenoidal and quadrupoles that are focusing in the $j$ plane, $f>0$, whereas for defocusing quadrupoles in the $j$ plane, $f<0$. 
General analytical solutions for the envelope evolution in drift regions are also unknown, though some simplifications can be made using envelope Hamiltonian invariants, in particular drift regions. However, the range of drift envelope evolution can be analytically understood in the extremes of an emittance-dominated beam with $Q=$ 0 and a space-charge dominated beam with $\varepsilon_{j}=0$. First, in the limit of a emittance-dominated beam, $Q=0$, and the $j$-plane envelope equation (4) reduces to

$$
r_{j}^{\prime \prime}-\frac{\varepsilon_{j}^{2}}{r_{j}^{3}}=0
$$

This equation is straightforward to integrate using the envelope Hamiltonian conservation constraint $r_{j}^{\prime 2} / 2+$ $\varepsilon_{j}^{2} /\left(2 r_{j}^{2}\right)=$ const. After some manipulations we obtain

$$
r_{j}(s)=r_{j}\left(s_{i}\right) \sqrt{1+\frac{2 r_{j}^{\prime}\left(s_{i}\right)}{r_{j}\left(s_{i}\right)}\left(s-s_{i}\right)+\left[1+\frac{r_{j}^{2}\left(s_{i}\right) r_{j}^{\prime 2}\left(s_{i}\right)}{\varepsilon_{j}^{2}}\right] \frac{\varepsilon_{j}^{2}}{r_{j}^{4}\left(s_{i}\right)}\left(s-s_{i}\right)^{2} .}
$$

Here, $s=s_{i}$ is the initial condition where $r_{j}=r_{j}\left(s_{i}\right)$ and $r_{j}^{\prime}=r_{j}^{\prime}\left(s_{i}\right)$. In the opposite limit of a space-charge dominated beam, $\varepsilon_{j}=0$, and the envelope equations can be cast in decoupled form using sum and difference coordinates defined as $r_{ \pm}=\left(r_{x} \pm r_{y}\right) / 2$ to give

$$
r_{+}^{\prime \prime}-\frac{Q}{r_{+}}=0, \quad r_{-}^{\prime \prime}=0
$$

The envelope Hamiltonian conservation constraint $r_{+}^{\prime 2} / 2-Q \ln r_{+}=$const can be employed to simplify integration of the equation for $r_{+}$and the equation for $r_{-}$is trivially integrable. We obtain (see Appendix D)

$r_{+}(s)=r_{+}\left(s_{i}\right) \exp \left(-\frac{r_{+}^{\prime 2}\left(s_{i}\right)}{2 Q}+\left[\operatorname{erfi}^{-1}\left\{\operatorname{erfi}\left[\frac{r_{+}^{\prime}\left(s_{i}\right)}{\sqrt{2 Q}}\right]+\sqrt{\frac{2 Q}{\pi}} e^{r_{+}^{\prime 2}\left(s_{i}\right) /(2 Q)} \frac{\left(s-s_{i}\right)}{r_{+}\left(s_{i}\right)}\right\}\right]^{2}\right), \quad r_{-}(s)=r_{-}\left(s_{i}\right)+r_{-}^{\prime}\left(s_{i}\right)\left(s-s_{i}\right)$.

Here, $\operatorname{erfi}(z)=\operatorname{erf}(i z) / i=(2 / \sqrt{\pi}) \int_{0}^{z} d t \exp \left(t^{2}\right)$ is the imaginary error function, $i \equiv \sqrt{-1}$, and the notation on the initial conditions is analogous to that employed in Eq. (16). An alternative form of the solution for $r_{+}$has been presented for axisymmetric beams by Humphries [16]. The solutions in Eqs. (18) can also be employed to parallel an axisymmetric beam analysis of O'Connell [17] to calculate the limiting density distribution of the free expansion of an elliptical beam.

To contrast these two limiting free-drift solutions, the envelope expansions $r_{x}(s) / r_{x}\left(s_{i}\right)$ and $r_{+}(s) / r_{ \pm}\left(s_{i}\right)$ given by Eqs. (16) and (18) are plotted in Fig. 3 for equal initial forces $Q / r_{+}\left(s_{i}\right)=\varepsilon_{x} / r_{x}^{3}\left(s_{i}\right)$ and zero initial angles. The space-charge dominated expansion is more rapid due to the $\sim 1 / r_{+}$expansion force being larger than the $\sim 1 / r_{x}^{3}$ expansion force at large radii. Equations (16) and (18) are useful to estimate the scaling of matched beam excursions in periodic focusing lattices (see discussions in Ref. [16]) and in calculating free drifts of a beam to a target or intercepting beam diagnostics. If a beam is allowed to expand freely for a long axial distance, the perveance term $\propto Q$ will eventually dominate the emittance term $\propto \varepsilon_{j}$ in the envelope equation (4), and Eq. (18) can be used to approximate the expansion in this regime.

\section{Single-particle phase advances}

Because it is useful to employ single-particle phase advances to parametrize matched solutions to the $\mathrm{KV}$ envelope equation (4), we review these phase advances here in order to define notations employed and summarize results needed later.

The solution to the linear $x$ equation of motion (1) can be expressed in terms of a transfer map as $[18,19]$

$$
\left(\begin{array}{c}
x(s) \\
x^{\prime}(s)
\end{array}\right)=\mathbf{M}_{x}\left(s \mid s_{i}\right) \cdot\left(\begin{array}{c}
x\left(s_{i}\right) \\
x^{\prime}\left(s_{i}\right)
\end{array}\right),
$$

where $\mathbf{M}_{x}\left(s \mid s_{i}\right)$ denotes the $2 \times 2$ transfer matrix from the initial $x$-plane particle phase-space coordinates at

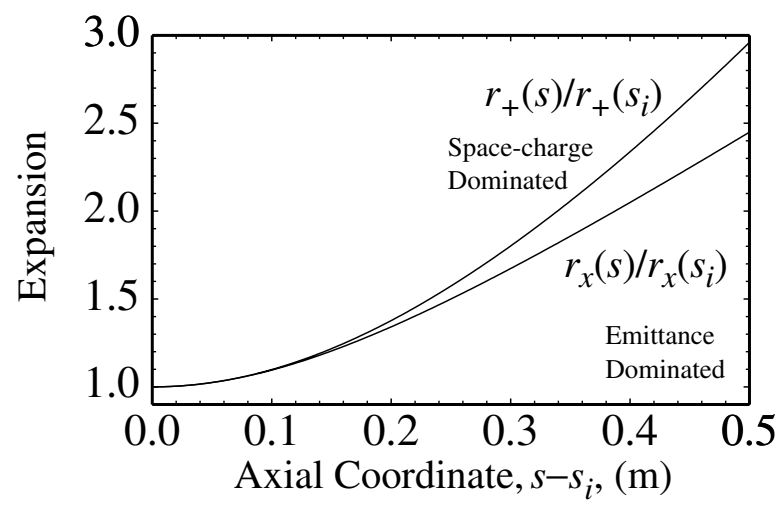

FIG. 3. Scaled envelope free expansions $r_{x}(s) / r_{x}\left(s_{i}\right)$ and $r_{+}(s) / r_{+}\left(s_{i}\right)$ plotted versus axial coordinate $s-s_{i}$. Curves correspond to the emittance-dominated $(Q=0)$ and spacecharge dominated $\left(\varepsilon_{x}=0\right)$ limits given by Eqs. (16) and (18). Initial coordinates at $s=s_{i}$ correspond to $Q / r_{+}\left(s_{i}\right)=$ $\varepsilon_{x} / r_{x}^{3}\left(s_{i}\right), Q=10^{-3}, r_{x}\left(s_{i}\right)=r_{+}\left(s_{i}\right)=0.01 \mathrm{~m}$, and $r_{x}^{\prime}\left(s_{i}\right)=$ $r_{+}^{\prime}\left(s_{i}\right)=0$. 
axial coordinate $s=s_{i}$ to the phase-space coordinates at $s$. An analogous equation holds for the $y$-plane orbit. An eigenvalue analysis of $\mathbf{M}_{j}\left(s \mid s_{i}\right)$ shows that orbit stability corresponds to

$$
\frac{1}{2}\left|\operatorname{Tr} \mathbf{M}_{j}\left(s_{i}+L_{p} \mid s_{i}\right)\right|<1,
$$

and that $\operatorname{Tr} \mathbf{M}_{j}\left(s_{i}+L_{p} \mid s_{i}\right)$ is independent of the initial axial coordinate $s_{i}$.

A stable orbit of the particle satisfying Eq. (1) can be expressed in terms of the phase-amplitude formulation as $[18,19]$

$$
\begin{aligned}
& x(s)=A_{x} w_{x}(s) \cos \left[\psi_{x}(s)\right], \\
& y(s)=A_{y} w_{y}(s) \cos \left[\psi_{y}(s)\right],
\end{aligned}
$$

where $A_{j}=$ const and the amplitude and phase functions $w_{j}$ and $\psi_{j}$ satisfy

$$
w_{j}^{\prime \prime}+\kappa_{j} w_{j}-\frac{2 Q w_{j}}{\left(r_{x}+r_{y}\right) r_{j}}-\frac{1}{w_{j}^{3}}=0, \quad \psi_{j}^{\prime}=\frac{1}{w_{j}^{2}} .
$$

For a periodic lattice with $\kappa_{j}\left(s+L_{p}\right)=\kappa_{j}(s)$, without loss of generality, $w_{j}$ can be taken to be the positive, real solution to the amplitude equation satisfying $w_{j}(s+$ $\left.L_{p}\right)=w_{j}(s)$. Comparing the amplitude equation (22) with the envelope equation (4) for a matched beam, we observe that $r_{j m}$ and $w_{j}$ are related as

$$
r_{j m}=\sqrt{\epsilon_{j}} w_{j}
$$

The phase advance $\sigma_{j}$ of the orbit through one lattice period can then unambiguously be defined by integrating $\psi_{j}^{\prime}=1 / w_{j}^{2}$ to yield

$$
\sigma_{j}=\psi_{j}\left(s_{i}+L_{p}\right)-\psi_{j}\left(s_{i}\right)=\varepsilon_{j} \int_{s_{i}}^{s_{i}+L_{p}} \frac{d s}{r_{j m}^{2}(s)},
$$

independent of $s_{i}$. Analysis of the transfer matrix elements then shows that

$$
\cos \sigma_{j}=\frac{1}{2} \operatorname{Tr} \mathbf{M}_{j}\left(s_{i}+L_{p} \mid s_{i}\right) .
$$

Direct application of this formula to calculate $\sigma_{j}$ requires proper branch identification.

For the case of an isotropic beam with $\varepsilon_{x}=\varepsilon_{y} \equiv \varepsilon$ and the three classes of lattices outlined in Sec. II B, the phase advances of the $x$ and $y$ orbits will be equal, i.e., $\sigma_{x}=\sigma_{y}$. In this symmetric situation, we will not distinguish between $\sigma_{x}$ and $\sigma_{y}$, and we denote $\sigma_{x}=\sigma_{y} \equiv \sigma$.

In the limit of zero space charge $(Q \rightarrow 0)$, we denote the phase advance $\sigma_{j}$ calculated from Eq. (24) as

$$
\sigma_{0 j} \equiv \lim _{Q \rightarrow 0} \sigma_{j}
$$

The so-called undepressed phase advance $\sigma_{0 j}$ measures the phase advance of particles in response to the linear applied focusing fields and is directly related to the focusing strength of the lattice. For periodic focusing lattices with $\kappa_{x}=\kappa_{y}$ or $\kappa_{x}=-\kappa_{y}, \sigma_{0 x}=\sigma_{0 y}$, and we denote $\sigma_{0 x}=\sigma_{0 y} \equiv \sigma_{0}$. Equations (1) and (25) can be employed to relate $\sigma_{0}=\lim _{Q \rightarrow 0} \sigma$ to the lattice focusing functions $\kappa_{j}$.

For the three classes of lattices described in Sec. II B, we summarize expressions for $\sigma_{0}$. First, for the continuous focusing lattice, $\kappa_{x}=\kappa_{y}=k_{\beta 0}^{2}=$ const and there is no strict periodicity. However, for purposes of comparisons to periodic systems (see Fig. 2), we can define the undepressed particle phase advance $\sigma_{0}$ over an arbitrary "period" $L_{p}$ by

$$
\sigma_{0}=k_{\beta 0} L_{p}
$$

For the hard-edge solenoidal and quadrupole doublet focusing channels illustrated in Fig. 2, it can be shown that

$$
\cos \sigma_{0}=\cos (2 \Theta)-\frac{1-\eta}{\eta} \Theta \sin (2 \Theta)
$$

for the solenoidal channel with $\Theta \equiv \sqrt{\hat{\kappa}} \eta L_{p} / 2$, and

$$
\begin{aligned}
\cos \sigma_{0}= & \cos \Theta \cosh \Theta \\
& +\frac{1-\eta}{\eta} \Theta(\cos \Theta \sinh \Theta-\sin \Theta \cosh \Theta) \\
& -2 \alpha(1-\alpha) \frac{(1-\eta)^{2}}{\eta^{2}} \Theta^{2} \sin \Theta \sinh \Theta
\end{aligned}
$$

for the quadrupole channel with $\Theta \equiv \sqrt{\left|\hat{\kappa}_{q}\right|} \eta L_{p} / 2$. For any periodic channel with specified focusing functions $\kappa_{j}(s)$ (including cases with fringe variations), particular values of $\sigma_{0}$ can be produced by scaling the amplitude of the focusing functions, i.e., $\kappa_{x} \rightarrow k \kappa_{x}$ and $\kappa_{y} \rightarrow k \kappa_{y}$ with $k=$ const constrained by $\sigma_{0}$. For example, consider the hard-edge quadrupole lattice. At specified lattice period $L_{p}$, occupancy $\eta$, and syncopation factor $\alpha$, Eq. (29) constrains the value of the focusing strength $\left|\hat{\kappa}_{q}\right|$ in terms of $\sigma_{0}$.

The thin-lens limit of Eqs. (28) and (29) can be explored by setting $\hat{\kappa}=1 /\left(\eta f L_{p}\right)$ for solenoids and $\hat{\kappa}_{q}=$ $2 /\left(\eta f L_{p}\right)$ for quadrupoles and then taking the limit $\eta \rightarrow$ 0 . This gives

$$
\cos \sigma_{0}= \begin{cases}1-\frac{1}{2} \frac{L_{p}}{f}, & \text { thin-lens solenoids } \\ 1-\frac{\alpha}{2}(1-\alpha)\left(\frac{L_{p}}{f}\right)^{2}, & \text { thin-lens quadrupoles. }\end{cases}
$$

These results can also be derived directly from a transfer matrix approach of the undepressed orbits using Eq. (25) in the limit $Q \rightarrow 0$ to show that 


$$
\begin{aligned}
\cos \sigma_{0}= & \frac{1}{2} \operatorname{Tr}\left[\begin{array}{cc}
1 & L_{p} \\
0 & 1
\end{array}\right]\left[\begin{array}{cc}
1 & 0 \\
-\frac{1}{f} & 1
\end{array}\right]=1-\frac{1}{2} \frac{L_{p}}{f}, \\
\cos \sigma_{0}= & \frac{1}{2} \operatorname{Tr}\left[\begin{array}{cc}
1 & 0 \\
-\frac{1}{f} & 1
\end{array}\right]\left[\begin{array}{cc}
1 & \alpha L_{p} \\
0 & 1
\end{array}\right]\left[\begin{array}{cc}
1 & 0 \\
\frac{1}{f} & 1
\end{array}\right] \\
& \times\left[\begin{array}{cc}
1 & (1-\alpha) L_{p} \\
0 & 1
\end{array}\right]=1-\frac{\alpha}{2}(1-\alpha)\left(\frac{L_{p}}{f}\right)^{2},
\end{aligned}
$$

for solenoidal and quadrupole channels, respectively.

When $\sigma_{0 x}=\sigma_{0 y}$ and $\sigma_{x}=\sigma_{y}$, the ratio of spacecharge depressed to undepressed phase advances $\sigma / \sigma_{0}$ provides a convenient, normalized measure of spacecharge intensity which we call the space-charge depression. For a fixed value of $\sigma_{0}$, the limit $\sigma / \sigma_{0} \rightarrow 0$ corresponds to a cold beam at the space-charge limit with zero emittance (i.e., $Q \neq 0$ and $\varepsilon=0$, or $Q \rightarrow \infty$ for finite $\varepsilon$ ), and the limit $\sigma / \sigma_{0} \rightarrow 1$ corresponds to an emittancedominated beam with zero space-charge effects (i.e., $\varepsilon \neq$ 0 with $Q=0$, or $\varepsilon \rightarrow \infty$ for finite $Q$ ). Stability properties of the envelope equation (4) will later be characterized in terms of $\sigma_{0}$ and $\sigma / \sigma_{0}$ for stable undepressed orbits with $\sigma_{0} \in(0, \pi)$ and the full range of space-charge intensity $\sigma / \sigma_{0} \in[0,1]$.

Matched envelopes can also exist in periodic transport channels for ranges (bands) of $\sigma_{0 j}$ with $\sigma_{0 j}>\pi$. These bands will correspond to higher focusing strength (e.g., larger $\hat{\kappa}$ for the hard-edge solenoidal lattice) and will be separated from the stable range $\sigma_{0 j} \in(0, \pi)$ by so-called stop bands where $(1 / 2)\left|\operatorname{Tr} \mathbf{M}_{j}\left(s_{i}+L_{p} \mid s_{i}\right)\right|>1$ and the single-particle orbits are unstable. Successive stable bands will have narrower ranges of $\sigma_{0 j}$ and the first stop band begins at $\sigma_{0 j}=\pi$. Higher stable bands tend to be more sparse and narrower for quadrupole focusing as opposed to solenoidal focusing, as can be seen from analyzing regions where $\left|\cos \sigma_{0}\right|<1$ from Eqs. (28) and (29). This occurs because stronger quadrupole focusing in alternating gradient lattices tends to produce large particle excursions within the defocusing quadrupoles, leading to narrow parameter bands for stable orbit bundles. Higher stable bands in solenoidal channels are wider than for quadrupole channels because such systems are focusing in all planes. However, contrary to assertions by some authors [20-22], we find that machine operation in higher bands of stability for solenoidal focusing will not, in general, prove advantageous. This follows because the higher bands require significantly larger focusing strengths, while for fixed beam perveance $Q$, these stronger optics also result in matched beams with larger envelope excursions than for $\sigma_{0 j}<\pi$, leading to larger apertures needed for beam confinement [23]. Moreover, matching the beam envelope at high phase advances can prove problematic when previous transport is at lower phase advances. Because of these problems, we consider only $\sigma_{0 j} \in(0, \pi)$ in this paper. Contrary to the case for $\sigma_{0 j}>\pi$, we find that for fixed $Q$ in periodic focusing lattices with high degrees of symmetry, that the matched beam envelope generally is radially more compact for higher values of $\sigma_{0 j}$ in the range $\sigma_{0 j} \in(0, \pi)[24,25]$.

\section{E. Scaled envelope equations and system parameters}

Solutions for the envelope equation (4) can be parametrized in terms of the lattice focusing functions $\kappa_{j}$, the perveance $Q$, and the emittances $\varepsilon_{j}$. For example, in the hard-edge solenoid and quadrupole doublet lattices defined in Sec. II B, the functions $\kappa_{j}$ are described in terms of the lattice period $L_{p}$, the occupancy $\eta$, the focusing strength $\hat{\kappa}$ (solenoids) or $\hat{\kappa}_{q}$ (quadrupoles), and the syncopation parameter $\alpha$ (quadrupoles only). Thus for hardedge solenoids, six “direct" parameters $\left(L_{p}, \eta, \hat{\kappa}, Q\right.$, and $\varepsilon_{j}$ ) can be employed to specify the matched beam solution, while for the hard-edge quadrupole doublet seven direct parameters $\left(L_{p}, \eta, \alpha, \hat{\kappa}_{q}, Q\right.$, and $\left.\varepsilon_{j}\right)$ can be employed. Other choices of hard-edge solenoidal and quadrupole lattices yield similar direct parameters. Focusing lattices including axial fringe field models of the elements introduce additional direct parameters that replace the hard-edge occupancy $\eta$ and focusing strengths $\hat{\kappa}$ and $\hat{\kappa}_{q}$.

Rather than employing the direct parameters, matched beam solutions of the envelope equations can be described by a reduced number of dimensionless parameters $[26,27]$. The particle phase advance parameters $\sigma_{0 j}$ and $\sigma_{j}\left(\right.$ or $\sigma_{0 j}$ and $\sigma_{j} / \sigma_{0 j}$ ) are convenient for this purpose. To simply illustrate this procedure we restrict the analysis to lattices with equal focusing strength $\left(\sigma_{0 x}=\sigma_{0 y} \equiv \sigma_{0}\right)$ and emittances $\left(\varepsilon_{x}=\varepsilon_{y} \equiv \varepsilon\right)$ in the $x$ and $y$ planes. It is straightforward to generalize this isotropic treatment for applications with anisotropic beams or focusing. First, we assume finite perveance $(Q>0)$ and for either continuous focusing or general periodic solenoidal or quadrupole transport lattices, we introduce scaled coordinates and express the envelope equation (4) as

$$
\frac{d^{2}}{d S^{2}} R_{j}+\xi_{j} F\left(S ; \sigma_{0},\{S\}\right) R_{j}-\frac{1}{R_{x}+R_{y}}-\frac{\Gamma^{2}}{R_{j}^{3}}=0 .
$$

Here, $S=s / L_{p}$ is a dimensionless axial coordinate that increases by unity as the beam advances over a lattice period $L_{p}$.

$$
R_{j}=\frac{r_{j}}{\sqrt{2 Q} L_{p}}
$$

are dimensionless scaled envelope radii, $\xi_{j}=1$ for continuous or solenoidal focusing, $\xi_{x}=-\xi_{y}=1$ for quadrupole focusing, $F\left(S ; \sigma_{0},\{S\}\right)$ is a scaled focusing function that depends on $S, \sigma_{0}$, and a set of "shape" parameters $\{S\}$ of the focusing function, and

$$
\Gamma=\frac{\varepsilon}{2 Q L_{p}}
$$

is a dimensionless parameter measuring relative 
space-charge intensity. For a matched beam envelope (i.e., $r_{j}=r_{j m}$ ), the parameter $\Gamma$ can be related to the depressed particle phase advance $\sigma$ through Eq. (24) as

$$
\sigma=\Gamma \int_{0}^{1} \frac{d S}{R_{j}^{2}(S)} .
$$

The parameters of the scaled envelope equation (31) are $\sigma_{0}$, the shape parameters $\{S\}$ which describe the action of the applied focusing forces on a single particle, and the dimensionless parameter $\Gamma$ defined in Eq. (34), or alternatively to $\Gamma, \sigma$, or $\sigma / \sigma_{0}$.

Specific examples of the scaled focusing function $F\left(S ; \sigma_{0},\{S\}\right)$ for the hard-edge solenoidal and quadrupole lattices described in Sec. II B clarify the forms given in Eqs. (31)-(34). For the solenoidal lattice, we take $F\left(S ; \sigma_{0},\{S\}\right)=F\left(S ; \sigma_{0}, \eta\right)=\left[L_{p}^{2} \hat{\kappa}\right]\left[\kappa_{x}(s) / \hat{\kappa}\right]$ where $\left[\kappa_{x}(s) / \hat{\kappa}\right]$ is a function of $S$ and $\eta$, and $\left[L_{p}^{2} \hat{\kappa}\right]$ is a function of $\sigma_{0}$ and $\eta$ through an application of the phase advance equation of constraint (28). Similarly, for the quadrupole lattice, we take $F\left(S ; \sigma_{0},\{S\}\right)=F\left(S ; \sigma_{0}, \eta, \alpha\right)=$ $\left[L_{p}^{2}\left|\hat{\kappa}_{q}\right|\right]\left[\kappa_{x}(s) /\left|\hat{\kappa}_{q}\right|\right]$, where $\left[\kappa_{x}(s) /\left|\hat{\kappa}_{q}\right|\right]$ is a function of $S, \eta$, and $\alpha$, and $\left[L_{p}^{2}\left|\hat{\kappa}_{q}\right|\right]$ is a function of $\sigma_{0}, \eta$, and $\alpha$ through an application of the phase advance equation of constraint (29). For the special case of continuous focusing, there are no shape parameters (i.e., $\{S\}=\varnothing$ ), and the choice of lattice period $L_{p}$ entering in $\sigma_{0}$ and $\sigma$ is arbitrary. Hence, matched solutions in continuous focusing can be regarded as a function solely of $\sigma / \sigma_{0}$ (see also Sec. III A).

From the results above, it follows for a beam with $\varepsilon_{x}=$ $\varepsilon_{y} \equiv \varepsilon$ that the matched envelope in the hard-edge solenoidal transport lattice considered can be described in terms of three parameters: the magnet occupancy $\eta$, the undepressed particle phase advance $\sigma_{0}$, and the spacecharge depression $\sigma / \sigma_{0}$ rather than five direct parameters [26]; whereas matched solutions to the quadrupole doublet lattice can be described in terms of four parameters: $\eta, \sigma_{0}, \sigma / \sigma_{0}$, plus the syncopation factor $\alpha(\alpha \equiv 1 / 2$ for the special case of a symmetric FODO lattice) rather than the five direct parameters [27]. In subsequent analyses we will exploit this reduction by specifying matched beam solutions in terms of these reduced parameter sets. However, to maintain a direct connection of the usual form of the envelope equations we will carry out most analyses in terms of conventional dimensional envelope coordinates and direct parameters.

\section{F. Small-amplitude perturbations about the matched beam envelope}

To analyze the evolution of perturbations about the matched beam envelope, we resolve the envelope coordinates $r_{j}$ evolving according to Eq. (4) from general initial conditions as

$$
r_{j}(s)=r_{j m}(s)+\delta r_{j}(s)
$$

Here, $r_{j m}$ is a matched solution to the envelope equation and $\delta r_{j}$ are perturbations about the matched beam envelope. In the small-amplitude limit we require that $\left|\delta r_{j}\right| \ll$ $r_{j m}$ over the range of $s$ analyzed. The envelope perturbations $\delta r_{j}$ are typically referred to as mismatch oscillations about the matched beam envelope.

In addition to the perturbations in the envelope coordinates $\delta r_{j}$, we consider additional driving perturbations that can excite the envelope perturbations from the matched beam condition. Specifically, we allow the lattice focusing functions $\kappa_{j}$, the beam perveance $Q$, and the rms emittances $\varepsilon_{j}$ to vary by making the following substitutions in the envelope equation (4):

$$
\begin{aligned}
\kappa_{j}(s) & \rightarrow \kappa_{j}(s)+\delta \kappa_{j}(s), \\
Q & \rightarrow Q+\delta Q(s), \\
\varepsilon_{j} & \rightarrow \varepsilon_{j}+\delta \varepsilon_{j}(s) .
\end{aligned}
$$

Here, $\delta \kappa_{j}(s)$ represent perturbations to the focusing functions $\kappa_{j}(s)$ used in generating the matched beam envelope, $\delta Q(s)$ with $-Q \leq \delta Q \leq 0$ represents the effect of particles lost outside the beam envelope, and $\delta \varepsilon_{j}(s)$ with $\varepsilon_{j}+\delta \varepsilon_{j}>0$ model evolutions in the rms emittances. The focusing perturbations $\delta \kappa_{j}(s)$ need not be periodic and can represent construction and excitation errors in the focusing optics. The long-path trend of the perturbed perveance $\delta Q(s) \leq 0$ will be negative due to halo particles being scraped, but it can locally increase if any halo particles reenter the core distribution. Similarly, over a long path the emittance perturbations $\delta \varepsilon_{j}(s)$ will tend to be positive and increasing due to the relaxation of the beam in response to nonlinear applied focusing fields, space-charge nonlinearities, etc. However, $\delta \varepsilon_{j}(s)$ can locally decrease because rms emittances are statistical measures of the phase-space area (e.g., unwrapping of strong " $\mathrm{S}$ "-shaped distortions in the particle phase space can reduce rms emittances). In the small-amplitude regime we require that $\delta Q \ll Q$ and that $\delta \kappa_{j}$ and $\delta \varepsilon_{j}$ be small in the sense that they induce small corrections on the particle orbits relative to $\kappa_{j}$ and $\varepsilon$, respectively. Higher-order theories outside the scope of the envelope model are generally necessary to calculate $\delta Q(s)$ and $\delta \varepsilon_{j}(s)$ for use in this formulation.

Inserting Eqs. (35) and (36) into the envelope equation (4) and neglecting all terms of order $\delta^{2}$ and higher yields the linearized perturbation equation

$$
\begin{aligned}
& \delta r_{j}^{\prime \prime}+\kappa_{j} \delta r_{j}+\frac{2 Q}{\left(r_{x m}+r_{y m}\right)^{2}}\left(\delta r_{x}+\delta r_{y}\right)+\frac{3 \varepsilon_{j}^{2}}{r_{j m}^{4}} \delta r_{j} \\
& =-r_{j m} \delta \kappa_{j}+\frac{2}{r_{x m}+r_{y m}} \delta Q+\frac{2 \varepsilon_{j}^{2}}{r_{j m}^{3}} \delta \varepsilon_{j} .
\end{aligned}
$$

This equation can be equivalently expressed in first-order matrix form as

$$
\frac{d}{d s} \delta \mathbf{R}+\mathbf{K} \cdot \delta \mathbf{R}=\delta \mathbf{P}
$$


where

$$
\delta \mathbf{R}=\left(\begin{array}{c}
\delta r_{x} \\
\delta r_{x}^{\prime} \\
\delta r_{y} \\
\delta r_{y}^{\prime}
\end{array}\right)
$$

is a vector of perturbed envelope phase-space coordinates, $\mathbf{K}$ is a $4 \times 4$ matrix with periodic $s$ dependence of period $L_{p}$ defined by

$$
\mathbf{K}=\left(\begin{array}{cccc}
0 & -1 & 0 & 0 \\
k_{x m} & 0 & k_{0 m} & 0 \\
0 & 0 & 0 & -1 \\
k_{0 m} & 0 & k_{y m} & 0
\end{array}\right)
$$

with

$$
k_{0 m}=\frac{2 Q}{\left(r_{x m}+r_{y m}\right)^{2}}, \quad k_{j m}=\kappa_{j}+3 \frac{\varepsilon_{j}^{2}}{r_{j m}^{4}}+k_{0 m},
$$

and

$$
\delta \mathbf{P}=\left(\begin{array}{c}
0 \\
-\delta \kappa_{x}+2 \frac{\delta Q}{r_{x m}+r_{y m}}+2 \frac{\varepsilon_{x} \delta \varepsilon_{x}}{r_{x m}^{3}} \\
0 \\
-\delta \kappa_{y}+2 \frac{\delta Q}{r_{x m}+r_{y m}}+2 \frac{\varepsilon_{y} \delta \varepsilon_{y}}{r_{y m}^{3}}
\end{array}\right)
$$

is a vector of driving perturbations. The solution to Eq. (38) can be expressed as

$$
\delta \mathbf{R}=\delta \mathbf{R}_{h}+\delta \mathbf{R}_{p}
$$

where $\delta \mathbf{R}_{h}$ is the general solution to the homogeneous equation (38) with $\delta \mathbf{P}=0$, and $\delta \mathbf{R}_{p}$ is any particular solution for the full equation with general $\delta \mathbf{P}$. Henceforth, the homogeneous and particular components of the solution will be analyzed in turn without explicitly distinguishing them to avoid cumbersome notation.

For the special cases of continuous $\left(\kappa_{x}=\kappa_{y}=k_{\beta 0}^{2}=\right.$ const) and periodic solenoidal focusing $\left(\kappa_{x}=\kappa_{y}=\kappa\right)$ channels with a round beam equilibrium $\left(\varepsilon_{x}=\varepsilon_{y}=\varepsilon\right.$ and $r_{x m}=r_{y m}=r_{m}$ ), considerable simplification results by resolving the envelope perturbations into sum and difference variables as

$$
\delta r_{ \pm} \equiv \frac{\delta r_{x} \pm \delta r_{y}}{2}
$$

In these cases, the perturbed envelope equation (37) can be expressed as

$$
\begin{gathered}
\delta r_{+}^{\prime \prime}+\kappa \delta r_{+}+\frac{2 Q}{r_{m}^{2}} \delta r_{+}+\frac{3 \varepsilon^{2}}{r_{m}^{4}} \delta r_{+}=-r_{m}\left(\frac{\delta \kappa_{x}+\delta \kappa_{y}}{2}\right)+\frac{1}{r_{m}} \delta Q+\frac{2 \varepsilon^{2}}{r_{m}^{3}}\left(\frac{\delta \varepsilon_{x}+\delta \varepsilon_{y}}{2}\right) \\
\delta r_{-}^{\prime \prime}+\kappa \delta r_{-}+\frac{3 \varepsilon^{2}}{r_{m}^{4}} \delta r_{-}=-r_{m}\left(\frac{\delta \kappa_{x}-\delta \kappa_{y}}{2}\right)+\frac{2 \varepsilon^{2}}{r_{m}^{3}}\left(\frac{\delta \varepsilon_{x}-\delta \varepsilon_{y}}{2}\right)
\end{gathered}
$$

and are decoupled. Here, $\kappa=k_{\beta 0}^{2}$ for continuous focusing. Each of the decoupled equations for $\delta r_{ \pm}$in Eq. (45) can be cast into a matrix form analogous to Eqs. (38)(43). However, in this case the vectors and matrices will be of dimension 2 rather than dimension 4. From the structure of Eq. (45), note that changes in beam perveance (i.e., $\delta Q \neq 0$ ) drive only $\delta r_{+}$and not $\delta r_{-}$, whereas changes in focusing strength $\left(\delta \kappa_{j} \neq 0\right)$ and emittance $\left(\delta \varepsilon_{j} \neq 0\right)$ can project on $\delta r_{+}$for in-phase error components in $x$ and $y$, and $\delta r_{-}$for out-of-phase errors. Also, due to the additional factor of $\left(2 Q / r_{m}^{2}\right) \delta r_{+}$, the restoring force coefficient $\propto \delta r_{ \pm}$is larger in the equation for $\delta r_{+}$than in the equation for $\delta r_{-}$, and oscillations in $\delta r_{+}$will be more rapid than oscillations in $\delta r_{-}$for $Q \neq 0$.

The homogeneous solution to the linear equation (38) describes the evolution of normal mode perturbations launched by mismatch in the initial conditions at $s=s_{i}$ (i.e., $\delta \mathbf{R} \neq 0$ at $s=s_{i}$ ), whereas the particular solution describes how mismatch oscillations can be driven by changes in beam and focusing parameters. The normal modes supported by the homogeneous equations have been previously analyzed for both continuous and periodic focusing channels by Struckmeier and Reiser [7]. In this paper, this previous work is extended to carefully identify classes of modes supported over a wide range of system parameters.

The homogeneous solution to Eq. (38) can be expressed in terms of a transfer map as

$$
\delta \mathbf{R}(s)=\mathbf{M}_{e}\left(s \mid s_{i}\right) \cdot \delta \mathbf{R}\left(s_{i}\right),
$$

where $\mathbf{M}_{e}\left(s \mid s_{i}\right)$ is a $4 \times 4$ matrix that maps the perturbed envelope coordinates $\delta \mathbf{R}$ from an initial axial coordinate $s=s_{i}$ to position $s$. Stability and launching conditions of normal modes can be analyzed in terms of eigenvalues and eigenvectors of the transfer map $\mathbf{M}_{e}$ $[18,19,28]$. The four complex eigenvectors $\mathbf{E}_{n}$ and eigenvalues $\lambda_{n}$ (not to be confused with the line charge $\lambda$ ) of the envelope transfer matrix through one lattice period $\mathbf{M}_{e}\left(s_{i}+L_{p} \mid s_{i}\right)$ from an initial condition $s_{i}$ are defined according to

$$
\mathbf{M}_{e}\left(s_{i}+L_{p} \mid s_{i}\right) \cdot \mathbf{E}_{n}\left(s_{i}\right)=\lambda_{n} \mathbf{E}_{n}\left(s_{i}\right),
$$

where $n=1,2,3$, and 4 . The overall scale of the eigenvectors $\mathbf{E}_{n}$ is arbitrary. Without loss of generality, we take all eigenvectors to be normalized according to $\mathbf{E}_{n} \cdot \mathbf{E}_{n}^{*}=$ 1 , where ${ }^{*}$ s denote complex conjugation. The eigenvalues 
$\lambda_{n}$ are independent of the location of the initial axial coordinate $s_{i}$, whereas the eigenvectors $\mathbf{E}_{n}\left(s_{i}\right)$ vary with the relative location of $s_{i}$ in the lattice period [28]. Stability in the sense of bounded excursions of perturbations in phase space correspond to $\lambda_{n}$ on the complex unit circle with $\left|\lambda_{n}\right|=1$ for all $n$, whereas instability results from any $\lambda_{n}$ lying off the complex unit circle (i.e., at least one unstable mode).

Because the envelope perturbation equation (38) is real and Hamiltonian, $\mathbf{M}_{e}$ is a real symplectic matrix. Consequently, the four eigenvalues $\lambda_{n}$ of $\mathbf{M}_{e}$ occur both as complex conjugate pairs $\left(\lambda_{n}, \lambda_{n}^{*}\right)$ and reciprocal pairs $\left(\lambda_{n}, 1 / \lambda_{n}\right)$ and can be categorized according to four symmetry classes (not counting possible degeneracies) described by Courant and Snyder [18] and Dragt [28]. These classes with associated eigenvalue and eigenvector symmetries are illustrated in Fig. 4. Eigenvalues are expressed in polar form as $\lambda_{n}=\gamma_{n} \exp \left(i \sigma_{n}\right)$ with real $\gamma_{n}$ and $\sigma_{n}$ and the specific labelings in $n$ indicated. Standard analyses $[18,19,28]$ show that $\gamma_{n}$ is the growth factor of
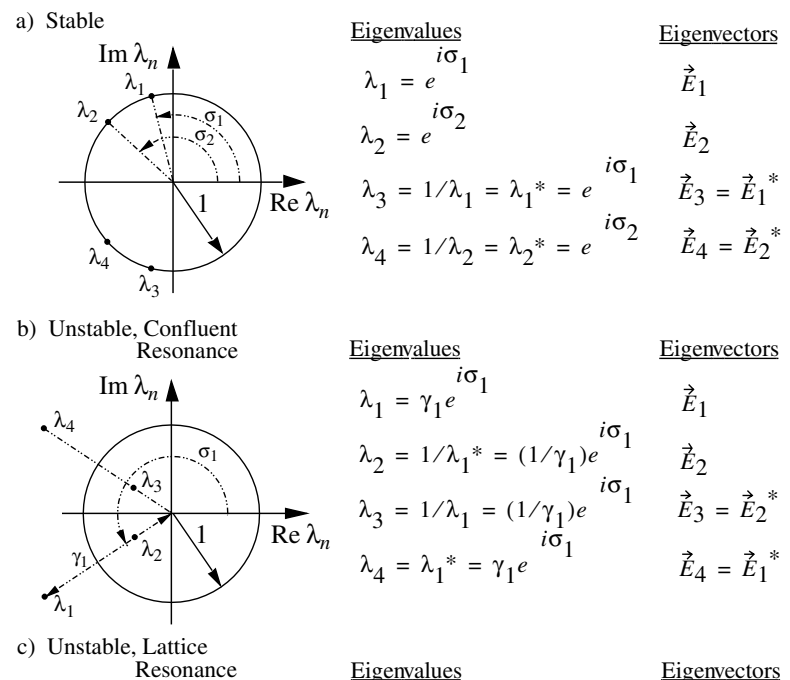

$$
\begin{array}{ll}
\text { Eigenvalues } & \text { Eigenvectors } \\
\lambda_{1}=\gamma_{1} e^{\sigma_{1}} & \vec{E}_{1} \\
\lambda_{2}=1 / \lambda_{1}{ }^{*}=\left(1 / \gamma_{1}\right) e^{i \sigma_{1}} & \vec{E}_{2} \\
\lambda_{3}=1 / \lambda_{1}=\left(1 / \gamma_{1}\right) e^{i \sigma_{1}} & \vec{E}_{3}=\vec{E}_{2}{ }^{*} \\
\lambda_{4}=\lambda_{1}{ }^{*}=\gamma_{1} e^{i \sigma_{1}} & \vec{E}_{4}=\vec{E}_{1}{ }^{*}
\end{array}
$$

c) Unstable, Lattice

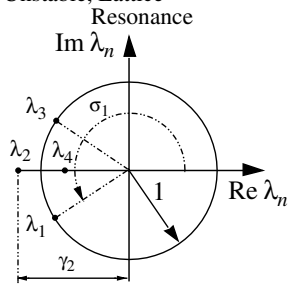

\section{Eigenvalues}

$\lambda_{1}=e^{i \sigma_{1}}$

$\lambda_{2}=\gamma_{2} e^{i \pi}$

$\lambda_{3}=\lambda_{1} *=e^{i \sigma_{1}}$

$\lambda_{4}=1 / \lambda_{2}=\left(1 / \gamma_{2}\right) e^{i \pi}$

Eigenvectors

$\vec{E}_{1}$

$\vec{E}_{2} \quad$ (real)

$\vec{E}_{3}=\vec{E}_{1}^{*}$

$\vec{E}_{4} \quad$ (real)

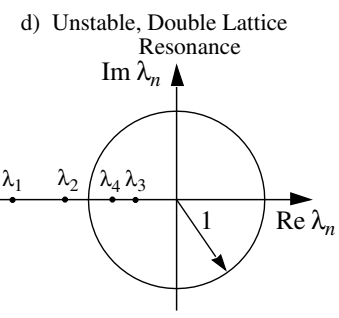

Eigenvalues

$\lambda_{1}=\gamma_{1} e^{i \pi}$

$\lambda_{2}=\gamma_{2} e^{i \pi}$

$\lambda_{3}=1 / \lambda_{1}=\left(1 / \gamma_{1}\right) e^{i \pi}$

$\lambda_{4}=1 / \lambda_{2}=\left(1 / \gamma_{2}\right) e^{i \pi}$

Eigenvectors

$\vec{E}_{1} \quad$ (real)

$\vec{E}_{2} \quad$ (real)

$\vec{E}_{3} \quad$ (real)

$\vec{E}_{4} \quad$ (real)

FIG. 4. Four possible classes of symmetries for the four eigenvalues defined by Eq. (47) corresponding to stability in (a), and instability in (b) $-(d)$. the mode per lattice period with $\left|\gamma_{n}\right|=1$ corresponding to stable oscillations, and $\left|\gamma_{n}\right|>1$ and $\left|\gamma_{n}\right|<1$ corresponding to oscillations with exponential growth and damping, respectively. The number of $e$-folds that the amplitude of mode oscillations grows per lattice period is $\ln \left|\gamma_{n}\right|$. It is shown later that $\sigma_{n}$ is related to the phase advance of the mode oscillations per lattice period. For each of the four classes of eigenvalue symmetries in Fig. 4, two real numbers are needed to describe the four eigenvalues [e.g., $\sigma_{1}$ and $\sigma_{2}$ in 4(a)]. In case 4(a), $\left|\lambda_{n}\right|=$ 1 and the eigenvectors corresponding to eigenvalues $\lambda_{n}$ and $1 / \lambda_{n}$ are simply related as complex conjugates with $\mathbf{E}_{3}=\mathbf{E}_{1}^{*}$ and $\mathbf{E}_{4}=\mathbf{E}_{2}^{*}$. For $\left|\lambda_{n}\right| \neq 1$, some relation between eigenvectors associated with the eigenvalues $\lambda_{n}$ and $1 / \lambda_{n}$ (i.e., through operations with $\mathbf{M}_{e}, \mathbf{M}_{e}^{-1}$, symplectic group generators, conjugation, etc.) would be useful rather than calculating them both directly from the characteristic equation defining the eigenvalues from Eq. (47). Unfortunately, it was shown that no such relationship exists in 4(c) and 4(d) [e.g., $\mathbf{E}_{1}$ and $\mathbf{E}_{3}$ are not simply related in 4(c)]. Although such a relationship may in principle exist in 4(b), efforts to derive it failed.

In Fig. 4, eigenvalues in case (a) correspond to stability $\left(\gamma_{n}=1\right)$, whereas cases (b)-(d) are unstable. Case 4(b) is called a "confluent resonance" instability because it represents a parametric resonance between both envelope mode oscillation frequencies and the lattice as evident by the eigenvalue symmetry. Figures 4 (c) and 4(d) are called "lattice resonance" and "double lattice resonance" instabilities because they represent a half-integer parametric resonance between the focusing structure and one or both mode oscillation frequencies. Transitions between cases lead to degenerate eigenvalues which can result in either linear growth in oscillation amplitude with periods traversed or no instability [28]. Which case the degeneracy falls into requires further analysis and is not addressed in this paper (other than a special case in the thin-lens limit) because the degenerate cases correspond to lower-dimensional surfaces in accessible parameter space that are launched with probability zero. For the types of lattices in Sec. II B, we find in Secs. III, IV, and $\mathrm{V}$ that the eigenvalues $\lambda_{n}$ fall into cases (a)-(c) and case (d) does not occur.

Any initial envelope perturbation at $s=s_{i}$ can be expanded in terms of the eigenvectors as

$$
\delta \mathbf{R}\left(s_{i}\right)=\sum_{n=1}^{4} \alpha_{n} \mathbf{E}_{n}\left(s_{i}\right)
$$

where the $\alpha_{n}$ are complex constants subject to $\delta \mathbf{R}\left(s_{i}\right)$ being real. To launch pure envelope modes with specific phase advances and growth factors for each of the eigenvalue classes listed in Fig. 4, it is convenient to resolve the initial condition $\delta R\left(s_{i}\right)=\delta R_{\ell}\left(s_{i}\right)$ into pure normal modes as summarized in Table I. Mode structures are illustrated in the table by mappings of the pure mode 
TABLE I. Launching conditions for distinct normal modes corresponding to the eigenvalue classes illustrated in Fig. 4. Here, $A_{\ell}$ and $\psi_{\ell}$ are real-valued amplitudes (unnormalized) and phases of mode $\ell(\ell=1,2$, and possibly 3 and 4 under conventions taken), and $\sigma_{\ell}$ and $\gamma_{\ell}>1$ are the phase advances and growth factors of the mode over one lattice period. Symbols are abbreviated with $\delta \mathbf{R}_{\ell} \equiv \delta \mathbf{R}_{\ell}\left(s_{i}\right), \mathbf{E}_{\ell} \equiv \mathbf{E}_{\ell}\left(s_{i}\right)$, and $\mathbf{M}_{e} \equiv \mathbf{M}_{e}\left(s_{i}+L_{p} \mid s_{i}\right)$.

\begin{tabular}{llll}
\hline \multicolumn{1}{c}{ Case } & \multicolumn{1}{c}{ Mode } & \multicolumn{1}{c}{ Launching condition } & \multicolumn{1}{c}{ Lattice period advance } \\
\hline (a) Stable & 1: Stable oscillation & $\delta \mathbf{R}_{1}=A_{1}\left(e^{\left.i \psi_{1} \mathbf{E}_{1}+e^{-i \psi_{1}} \mathbf{E}_{1}^{*}\right)}\right.$ & $\mathbf{M}_{e} \delta \mathbf{R}_{1}\left(\psi_{1}\right)=\delta \mathbf{R}_{1}\left(\psi_{1}+\sigma_{1}\right)$ \\
& 2: Stable oscillation & $\delta \mathbf{R}_{2}=A_{2}\left(e^{i \psi_{2}} \mathbf{E}_{2}+e^{-i \psi_{2}} \mathbf{E}_{2}^{*}\right)$ & $\mathbf{M}_{e} \delta \mathbf{R}_{2}\left(\psi_{2}\right)=\delta \mathbf{R}_{2}\left(\psi_{2}+\sigma_{2}\right)$ \\
(b) Unstable & 1: Exponential growth & $\delta \mathbf{R}_{1}=A_{1}\left(e^{i \psi_{1}} \mathbf{E}_{1}+e^{\left.-i \psi_{1} \mathbf{E}_{1}^{*}\right)}\right.$ & $\mathbf{M}_{e} \delta \mathbf{R}_{1}\left(\psi_{1}\right)=\gamma_{1} \delta \mathbf{R}_{1}\left(\psi_{1}+\sigma_{1}\right)$ \\
confluent resonance & 2: Exponential damping & $\delta \mathbf{R}_{2}=A_{2}\left(e^{i \psi_{2}} \mathbf{E}_{2}+e^{-i \psi_{2}} \mathbf{E}_{2}^{*}\right)$ & $\mathbf{M}_{e} \delta \mathbf{R}_{2}\left(\psi_{2}\right)=\left(1 / \gamma_{1}\right) \delta \mathbf{R}_{2}\left(\psi_{2}+\sigma_{1}\right)$ \\
(c) Unstable & 1: Stable oscillation & $\delta \mathbf{R}_{1}=A_{1}\left(e^{i \psi_{1}} \mathbf{E}_{1}+e^{-i \psi_{1}} \mathbf{E}_{1}^{*}\right)$ & $\mathbf{M}_{e} \delta \mathbf{R}_{1}\left(\psi_{1}\right)=\delta \mathbf{R}_{1}\left(\psi_{1}+\sigma_{1}\right)$ \\
lattice resonance & 2: Exponential growth & $\delta \mathbf{R}_{2}=A_{2} \mathbf{E}_{2}$ & $\mathbf{M}_{e} \delta \mathbf{R}_{2}=-\gamma_{2} \delta \mathbf{R}_{2}$ \\
& 3: Exponential damping & $\delta \mathbf{R}_{3}=A_{3} \mathbf{E}_{4}$ & $\mathbf{M}_{e} \delta \mathbf{R}_{3}=-\left(1 / \gamma_{2}\right) \delta \mathbf{R}_{3}$ \\
(d) Unstable & 1: Exponential growth & $\delta \mathbf{R}_{1}=A_{1} \mathbf{E}_{1}$ & $\mathbf{M}_{e} \delta \mathbf{R}_{1}=-\gamma_{1} \delta \mathbf{R}_{1}$ \\
double lattice & 2: Exponential growth & $\delta \mathbf{R}_{2}=A_{2} \mathbf{E}_{2}$ & $\mathbf{M}_{e} \delta \mathbf{R}_{2}=-\gamma_{2} \delta \mathbf{R}_{2}$ \\
resonance & 3: Exponential damping & $\delta \mathbf{R}_{3}=A_{3} \mathbf{E}_{3}$ & $\mathbf{M}_{e} \delta \mathbf{R}_{3}=-\left(1 / \gamma_{1}\right) \delta \mathbf{R}_{3}$ \\
& 4: Exponential damping & $\delta \mathbf{R}_{4}=A_{4} \mathbf{E}_{4}$ & $\mathbf{M}_{e} \delta \mathbf{R}_{4}=-\left(1 / \gamma_{2}\right) \delta \mathbf{R}_{4}$ \\
\hline \hline
\end{tabular}

initial condition through one lattice period. The eigenvectors $\mathbf{E}_{n}$, and consequently the pure mode launching conditions $\delta \mathbf{R}_{\ell}\left(s_{i}\right)$, vary with the relative location of the initial condition $s_{i}$ in the lattice period. Note that cases in Figs. 4(a) and 4(b) have two distinct modes, while cases in Figs. 4(c) and 4(d) have three and four distinct modes.

The evolution of the beam mismatch $\delta \mathbf{R}(s)$ can also be interpreted as evolving linear projections of envelope mismatch onto pure normal modes according to the cases in Table I as

$$
\delta \mathbf{R}(s)= \begin{cases}A_{1}\left[\mathbf{E}_{1}(s) e^{i \psi_{1}(s)}+\mathbf{E}_{1}^{*}(s) e^{-i \psi_{1}(s)}\right]+A_{2}\left[\mathbf{E}_{2}(s) e^{i \psi_{2}(s)}+\mathbf{E}_{2}^{*}(s) e^{-i \psi_{2}(s)}\right], & \text { cases (a), (b), } \\ A_{1}\left[\mathbf{E}_{1}(s) e^{i \psi_{1}(s)}+\mathbf{E}_{1}^{*}(s) e^{-i \psi_{1}(s)}+A_{2} \mathbf{E}_{2}(s)+A_{3} \mathbf{E}_{4}(s),\right. & \text { case (c), } \\ A_{1} \mathbf{E}_{1}(s)+A_{2} \mathbf{E}_{2}(s)+A_{3} \mathbf{E}_{3}(s)+A_{4} \mathbf{E}_{4}(s), & \text { case (d), }\end{cases}
$$

where $A_{\ell}$ and $\psi_{\ell}$ are real valued. This interpretation allows us to unambiguously define the pure mode phase advance $\sigma_{\ell}$ as the change in $\psi_{\ell}$ as $s$ advances over one lattice period $L_{p}$, thereby providing a concrete branch selection criteria to relate the complex eigenvalues $\lambda_{n}$ to the mode phase advances $\sigma_{\ell}$. Alternatively, the correct branch for the mode phase advance $\sigma_{\ell}$ can be selected by numerically integrating Eq. (38) with $\delta \mathbf{P}=0$ from the pure mode initial conditions in Table I to calculate the orbit $\delta \mathbf{R}_{\ell}(s)$, and then Fourier transforming $\delta r_{x}(s)$ and $\delta r_{y}(s)$ to identify frequency components. A sufficient propagation distance should be taken to allow clear branch identification from discretized transforms.

A specific initial envelope mismatch $\delta \mathbf{R}\left(s_{i}\right)$ can be resolved into normal mode projections according to Eq. (49) by solving for the four (in total) real-valued amplitude $A_{\ell}$ and phase $\psi_{\ell}\left(s_{i}\right)$ parameters needed. Values of $A_{\ell} \cos \psi_{\ell}\left(s_{i}\right)$ and $A_{\ell} \sin \psi_{\ell}\left(s_{i}\right)$ needed $\left[\psi_{\ell}=\pi\right.$ for $\ell=2,3$ in case (c) and for all $\ell$ in case (d)] can be found by matrix inversion of Eq. (49). A pure mode of a given amplitude can be launched by setting one $A_{\ell}$ in Eq. (49) to the desired amplitude and setting all other $A_{\ell}=0$. The pure mode phase $\psi_{\ell}$ can be varied over $2 \pi$ [except modes that do not have phases: $\ell=2,3$ in case (c) and all $\ell$ in case (d)] to sweep through all possible phase launches. Individual mode launching conditions are not, in general, orthogonal [e.g., $A_{1}\left(\mathbf{E}_{1} e^{i \psi_{1}}+\mathbf{E}_{1}^{*} e^{-i \psi_{1}}\right)$.
$A_{2}\left(\mathbf{E}_{2} e^{i \psi_{2}}+\mathbf{E}_{2}^{*} e^{-i \psi_{2}}\right) \neq 0$ in case (a)]. However, in the stable case (a) the two modes are almost orthogonal in a sense that $\lim _{L \rightarrow \infty}(1 / L) \int_{0}^{L} d s A_{1}\left[\mathbf{E}_{1} e^{i \psi_{1}}+\mathbf{E}_{1}^{*} e^{-i \psi_{1}}\right]$. $A_{2}\left[\mathbf{E}_{2} e^{i \psi_{2}}+\mathbf{E}_{2}^{*} e^{-i \psi_{2}}\right]=0$.

For the special cases of continuous and periodic solenoidal focusing channels with a round beam equilibrium $\left(\varepsilon_{x}=\varepsilon_{y}=\varepsilon\right.$ and $\left.r_{x m}=r_{y m}=r_{m}\right)$, the mode structure is simpler to analyze in terms of the decoupled equations (45) for $\delta r_{ \pm}=\left(\delta r_{x} \pm \delta r_{y}\right) / 2$ rather than the coupled equation (37) for $\delta r_{x}$ and $\delta r_{y}$. The transfer matrix $\mathbf{M}_{e}$ that advances $\delta \mathbf{R}=\left(\delta r_{+}, \delta r_{+}^{\prime}, \delta r_{-}, \delta r_{-}^{\prime}\right)$ through one lattice period is of block diagonal form with

$$
\mathbf{M}_{e}\left(s_{i}+L_{p} \mid s_{i}\right)=\left[\begin{array}{cc}
\mathbf{M}_{+}\left(s_{i}+L_{p} \mid s_{i}\right) & 0 \\
0 & \mathbf{M}_{-}\left(s_{i}+L_{p} \mid s_{i}\right)
\end{array}\right],
$$

where $\mathbf{M}_{ \pm}\left(s_{i}+L_{p} \mid s_{i}\right)$ are $2 \times 2$ symplectic matrices that can be independently analyzed for the stability of $\delta \mathbf{R}_{ \pm}=\left(\delta r_{ \pm}, \delta r_{ \pm}^{\prime}\right)$. The analysis for the normal modes and launching conditions supported by the uncoupled homogeneous equations is analogous to the coupled case above. For each uncoupled equation for $\delta r_{ \pm}$we need only analyze a $2 \times 2$ eigenvalue equation

$$
\mathbf{M}_{ \pm}\left(s_{i}+L_{p} \mid s_{i}\right) \cdot \mathbf{E}_{n}\left(s_{i}\right)=\lambda_{ \pm} \mathbf{E}_{n}\left(s_{i}\right) .
$$


Each of these reduced equations defines a pair of eigenvalues: $\lambda_{+}$and $1 / \lambda_{+}$for the $\delta r_{+}$equation, and $\lambda_{-}$and $1 / \lambda_{-}$for the $\delta r_{-}$equation. Stable modes with $\lambda_{ \pm}$on the unit circle correspond to [19]

$$
\frac{1}{2}\left|\operatorname{Tr} \mathbf{M}_{ \pm}\left(s_{i}+L_{p} \mid s_{i}\right)\right|<1 .
$$

Possible reduced symmetry classes of the eigenvalues are illustrated in Fig. 5 [28]. It follows that for a continuous or periodic solenoidal focusing channel with a round beam equilibrium there can be only a single stable mode (phase advance $\sigma_{ \pm}$) or two modes: one with pure exponential growth, and one with pure damping (both of the lattice resonance classes with phase advance $\sigma_{ \pm}=\pi$ ). The situ- ation in Fig. 4(b) corresponding to a confluent resonance is excluded when the $4 \times 4$ coupled envelope equations reduce to two $2 \times 2$ decoupled equations. Initial envelope perturbations in $x$ and $y$ can be projected onto the sum and difference perturbations at axial coordinate $s=s_{i}$ as

$$
\begin{aligned}
& \delta r_{x}\left(s_{i}\right)=\delta r_{+}\left(s_{i}\right)+\delta r_{-}\left(s_{i}\right), \\
& \delta r_{x}^{\prime}\left(s_{i}\right)=\delta r_{+}^{\prime}\left(s_{i}\right)+\delta r_{-}^{\prime}\left(s_{i}\right), \\
& \delta r_{y}\left(s_{i}\right)=\delta r_{+}\left(s_{i}\right)-\delta r_{-}\left(s_{i}\right), \\
& \delta r_{y}^{\prime}\left(s_{i}\right)=\delta r_{+}^{\prime}\left(s_{i}\right)-\delta r_{-}^{\prime}\left(s_{i}\right) .
\end{aligned}
$$

For pure mode launches, one takes

$$
\begin{aligned}
& \delta r_{-}\left(s_{i}\right)=0=\delta r_{-}^{\prime}\left(s_{i}\right), \quad \delta r_{+}\left(s_{i}\right) \neq 0, \text { and } / \text { or } \delta r_{+}^{\prime}\left(s_{i}\right) \neq 0 \text { : breathing mode, } \\
& \delta r_{+}\left(s_{i}\right)=0=\delta r_{+}^{\prime}\left(s_{i}\right), \quad \delta r_{-}\left(s_{i}\right) \neq 0, \text { and } / \text { or } \delta r_{-}^{\prime}\left(s_{i}\right) \neq 0 \text { : quadrupole mode. }
\end{aligned}
$$

This launching condition is schematically plotted in Fig. 6. Note that the pure mode in $\delta r_{+}$with $\delta r_{-}=0$ has in-phase perturbations with $\delta r_{x}=\delta r_{y}$ (breathing distortion), whereas a pure mode in $\delta r_{-}$with $\delta r_{+}=0$ has $\pi$ out-of-phase perturbations with $\delta r_{x}=-\delta r_{y}$ (quadrupole distortion). For this reason, pure decoupled modes in $\delta r_{+}$and $\delta r_{-}$are often called "breathing" and "quadrupole" modes, respectively.

a) Stable

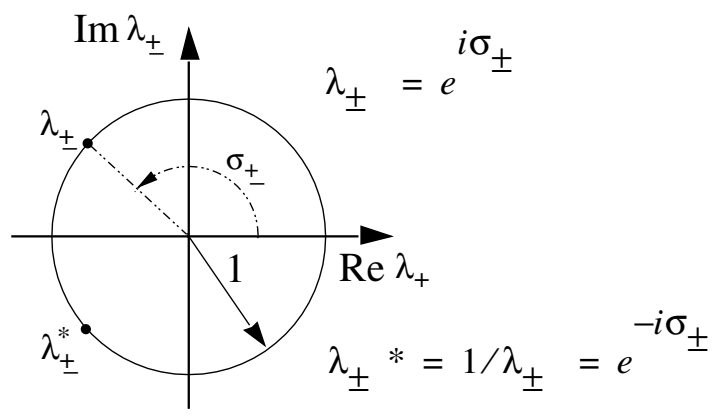

b) Unstable, Lattice

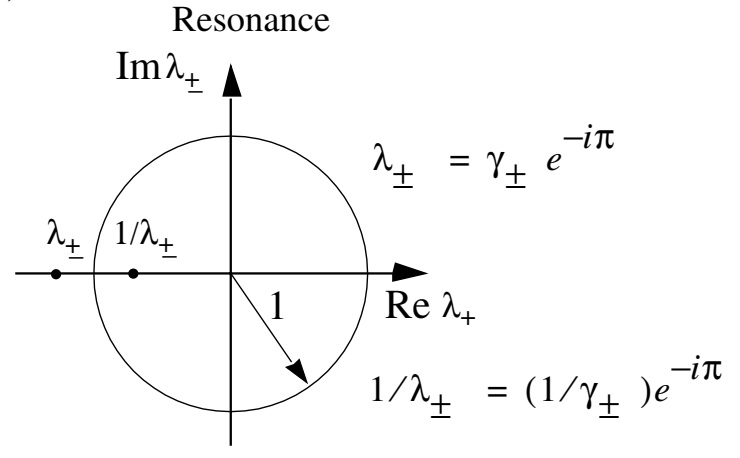

FIG. 5. Two possible classes of eigenvalue symmetries for continuous focusing or periodic solenoidal focusing channels with round beam equilibrium corresponding to stability in (a), and instability in (b).
In the limit of zero space charge $(Q \rightarrow 0)$, the perturbed envelope equation (37) decouples and the envelope modes can be analyzed independently in the $x$ and $y$ planes. Therefore, eigenvalue cases for undriven modes reduce to decoupled $2 \times 2$ problems in the $\delta r_{j}$ and $\delta r_{j}^{\prime}$ variables that are directly analogous to the cases discussed above for the $\delta r_{ \pm}$and $\delta r_{ \pm}^{\prime}$ variables. A phaseamplitude analysis is employed in Appendix E to show that in this zero space-charge limit the phase advance $\sigma_{e j}$ of oscillations in $\delta r_{j}$ over one lattice period is

$$
\sigma_{e j}=2 \sigma_{0 j}
$$

This limit holds for any periodic focusing functions $\kappa_{j}(s)$. An immediate consequence of Eq. (55) is that all coordinates connected by linear transformation to the $\delta r_{j}$ and $\delta r_{j}^{\prime}$ coordinates must have oscillations with the same phase advance. Normal mode coordinates $\delta \mathbf{R}_{\ell}$ of an envelope mode are related to the $\delta r_{j}$ and $\delta r_{j}^{\prime}$ coordinates through linear transformations specified by the eigenvectors as given in Table I. Therefore, the phase advance $\sigma_{\ell}$ of any normal envelope mode for any transport channel with $\sigma_{0 j}=\sigma_{0}$ and a symmetric beam with $\varepsilon_{x}=\varepsilon_{y}$ must satisfy the zero space-charge limit

$$
\lim _{Q \rightarrow 0} \sigma_{\ell}=2 \sigma_{0},
$$

regardless of the details of the focusing lattice. This limit provides a consistency check useful in practical calculations. The result can be understood qualitatively as follows. All $j$-plane particle oscillations in the zero spacecharge limit beam have phase advance $\sigma_{0 j}$. Particles contributing to the perturbed beam edge in phase space must have contributions from positive and negative coordinates, leading to an envelope locus of particle orbits that oscillates at double the particle oscillation frequency. A consequence of the limit (56) and the decoupling of the $x$ and $y$ coordinates for zero space charge is that envelope instabilities for zero space charge can only occur for $\sigma_{0}=\pi / 2$ or $\sigma_{0}=\pi$. This can be understood as follows. 


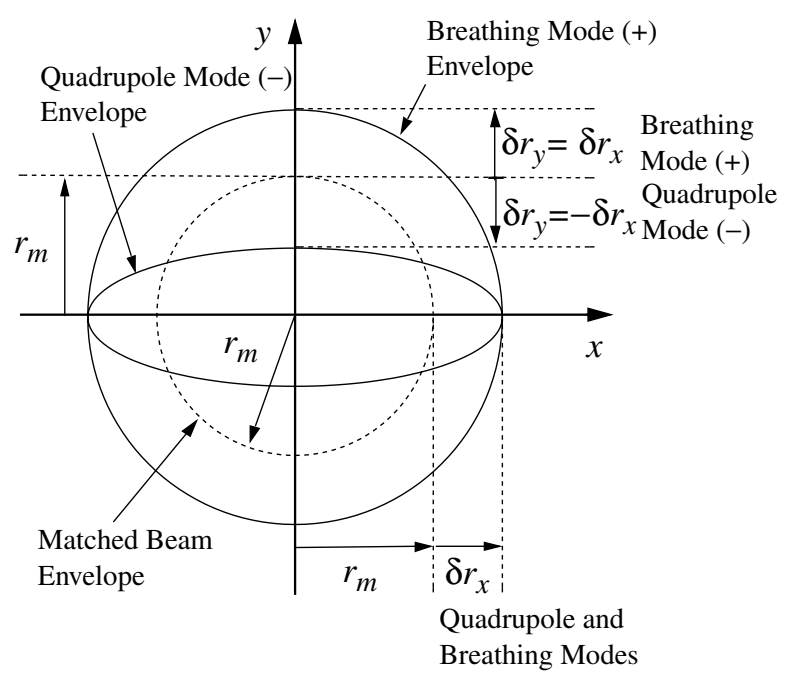

FIG. 6. Round, matched beam envelope with $r_{x}=r_{y}=r_{m}$ in a continuous or solenoidal focusing channel with pure breathing $\left(\delta r_{x}=\delta r_{y}\right)$ and quadrupole $\left(\delta r_{x}=-\delta r_{y}\right)$ mode perturbations superimposed.

Because the coordinates are decoupled, only lattice resonance instabilities [see Fig. 5(b)] are possible in the $x$ and $y$ variables or in any other set of variables connected to these by linear transformations. Therefore, for instability, the eigenvalues must lie on the real axis and $\lim _{Q \rightarrow 0} \sigma_{\ell}$ must be an integer multiple of $\pi$. Equation (56) then establishes a necessary (but not sufficient) condition for instability in that $\sigma_{0}$ be an integer multiple of $\pi / 2$, of which only $\pi / 2$ and $\pi$ are relevant to the analysis in this paper because we consider only stable undepressed particle orbits with $\sigma_{0}<\pi$.

Another feature of the envelope normal modes is that they evolve with quadratic Courant-Snyder invariants in the normal coordinate phase-space variables of the modes. These invariants are valid regardless of the details of the lattice focusing functions $\kappa_{j}(s)$ and can be interpreted as measures of phase-space area in the normal coordinates of the envelope modes. In Appendix F, a complete derivation of the envelope Courant-Snyder invariants is presented for the case of envelope perturbations in continuous and solenoidal focusing channels with $\kappa_{x}=\kappa_{y} \equiv \kappa$ and a symmetric matched beam with $\varepsilon_{x}=$ $\varepsilon_{y}$. In this case the normal coordinates of the envelope mode perturbations are the simple sum and difference coordinates $\delta r_{ \pm}=\left(r_{x} \pm r_{y}\right) / 2$ which evolve according to Eq. (45) with zero driving terms. This situation renders the derivation of the invariants directly analogous to standard treatments for decoupled single-particle orbits and shows that

$$
\left(\frac{\delta r_{ \pm}}{w_{ \pm}}\right)^{2}+\left(w_{ \pm}^{\prime} \delta r_{ \pm}-w_{ \pm} \delta r_{ \pm}^{\prime}\right)^{2}=\mathrm{const}
$$

where the $w_{ \pm}$are positive mode amplitude functions satisfying

$$
\begin{array}{r}
w_{+}^{\prime \prime}+\kappa w_{+}+\frac{2 Q}{r_{m}^{2}} w_{+}+\frac{3 \varepsilon^{2}}{r_{m}^{4}} w_{+}-\frac{1}{w_{+}^{3}}=0 \\
w_{-}^{\prime \prime}+\kappa w_{-}+\frac{3 \varepsilon^{2}}{r_{m}^{4}} w_{-}-\frac{1}{w_{-}^{3}}=0
\end{array}
$$

For stable envelope oscillations, the amplitudes $w_{ \pm}$can be interpreted as scaled maximum excursions of pure envelope modes. Analogous Courant-Snyder invariants exist also for the more complicated case of lattices with $\kappa_{x} \neq$ $\kappa_{y}$, where the coupled envelope perturbations $\delta r_{j}$ evolve according to Eq. (37) with zero driving terms. In this case, calculation of normal mode coordinates is much more complicated and is treated in Appendix F by connecting the formulation to a reference where the general coupling problem is analyzed. Although envelope mode Courant-Snyder invariants increase our formal understanding of the mode structure, in practical applications the utility of these invariants is limited because they require the calculation of periodic amplitude functions in the lattice that are not, in general, analytically tractable. However, for a given lattice and beam parameters, these amplitude functions can be numerically calculated and the mode invariants can then be employed to improve understanding of mode evolution and launching conditions in an analogous manner to standard treatments of single-particle dynamics.

Finally, the particular solution to Eq. (38) for driving perturbations with $\delta \mathbf{P} \neq 0$ is complicated for periodic focusing lattices. In Sec. III we construct the particular solution for the special case of continuous focusing where it can be carried out directly. This special case may provide a partial guide to the much more complicated situation for periodic focusing lattices which is not addressed in this paper. However, future studies of the particular solution will be aided from the analyses of the homogeneous solutions for periodic solenoidal and quadrupole focusing lattices presented in Secs. IV and $\mathrm{V}$, because such solutions can aid in formulation of Green's function type methods to construct the particular solution.

\section{ENVELOPE MODES IN CONTINUOUS FOCUSING CHANNELS}

The continuous focusing model described in Sec. II B is straightforward to analyze and can serve as a good approximation to more realistic periodic focusing lattices when effects do not depend strongly on the periodic nature of the applied focusing forces. In this regard, we find in Secs. IV and V that the continuous focusing model can provide good estimates of normal mode oscillation frequencies in periodic lattices when system parameters are far from bands of parametric instability. Moreover, the continuous focusing model can be solved exactly for driven envelope perturbations ( $\delta \mathbf{P} \neq 0)$, thereby providing new insight into this previously unexplored situation. 


\section{A. Matched envelope solution}

In a continuous focusing channel, $\kappa_{x}=\kappa_{y}=k_{\beta 0}^{2}=$ $\left(\sigma_{0} / L_{p}\right)^{2}$ [see Eq. (27)]. In this situation, if $\varepsilon_{x}=\varepsilon_{y}=$ $\varepsilon$, then the matched beam envelope is round with $r_{x m}=$ $r_{y m} \equiv r_{m}=$ const, where $r_{m}$ satisfies the reduced envelope equation of constraint

$$
k_{\beta 0}^{2} r_{m}-\frac{Q}{r_{m}}-\frac{\varepsilon^{2}}{r_{m}^{3}}=0
$$

or equivalently,

$$
\left(\frac{r_{m}}{L_{p}}\right)^{2}=\frac{Q}{2 \sigma_{0}^{2}}\left[1+\sqrt{1+4 \frac{\sigma_{0}^{2} \varepsilon^{2}}{Q^{2} L_{p}^{2}}}\right] .
$$

From Eqs. (24) and (60), the depressed phase advance of a particle over an axial distance $L_{p}$ is given by

$$
\sigma=\sqrt{\sigma_{0}^{2}-\frac{Q}{\left(r_{m} / L_{p}\right)^{2}}}=\frac{\varepsilon L_{p}}{r_{m}^{2}} .
$$

Equations (60) and (61) can be used to relate the spacecharge depression $\sigma / \sigma_{0}$ for the continuous focused beam, which is independent of the specific choice of $L_{p}$, to the beam parameters as

$$
\frac{k_{\beta 0}^{2} \varepsilon^{2}}{Q^{2}}=\frac{\sigma_{0}^{2} \varepsilon^{2}}{Q^{2} L_{p}^{2}}=\frac{\left(\sigma / \sigma_{0}\right)^{2}}{\left[1-\left(\sigma / \sigma_{0}\right)^{2}\right]^{2}} .
$$

\section{B. Envelope modes}

Using the equilibrium condition in Eq. (61), the linearized equations (45) describing small-amplitude elliptical beam perturbations $\delta r_{ \pm}=\left(\delta r_{x} \pm \delta r_{y}\right) / 2$ in the continuous focusing model can be expressed in scaled form as

$$
\begin{aligned}
& L_{p}^{2} \frac{d^{2}}{d s^{2}}\left(\frac{\delta r_{+}}{r_{m}}\right)+\sigma_{+}^{2}\left(\frac{\delta r_{+}}{r_{m}}\right)=-\frac{\sigma_{0}^{2}}{2}\left(\frac{\delta \kappa_{x}}{k_{\beta 0}^{2}}+\frac{\delta \kappa_{y}}{k_{\beta 0}^{2}}\right)+\left(\sigma_{0}^{2}-\sigma^{2}\right) \frac{\delta Q}{Q}+\sigma^{2}\left(\frac{\delta \varepsilon_{x}}{\varepsilon}+\frac{\delta \varepsilon_{y}}{\varepsilon}\right), \\
& L_{p}^{2} \frac{d^{2}}{d s^{2}}\left(\frac{\delta r_{-}}{r_{m}}\right)+\sigma_{-}^{2}\left(\frac{\delta r_{-}}{r_{m}}\right)=-\frac{\sigma_{0}^{2}}{2}\left(\frac{\delta \kappa_{x}}{k_{\beta 0}^{2}}-\frac{\delta \kappa_{y}}{k_{\beta 0}^{2}}\right)+\sigma^{2}\left(\frac{\delta \varepsilon_{x}}{\varepsilon}-\frac{\delta \varepsilon_{y}}{\varepsilon}\right),
\end{aligned}
$$

where

$$
\sigma_{+} \equiv \sqrt{2 \sigma_{0}^{2}+2 \sigma^{2}}, \quad \sigma_{-} \equiv \sqrt{\sigma_{0}^{2}+3 \sigma^{2}} .
$$

The homogeneous solutions to Eq. (63) are trivially expressible as

$$
\delta r_{ \pm}(s)=\delta r_{ \pm}\left(s_{i}\right) \cos \left(\sigma_{ \pm} \frac{s-s_{i}}{L_{p}}\right)+\frac{\delta r_{ \pm}^{\prime}\left(s_{i}\right)}{\sigma_{ \pm} / L_{p}} \sin \left(\sigma_{ \pm} \frac{s-s_{i}}{L_{p}}\right)
$$

where $r_{ \pm}\left(s_{i}\right)$ and $r_{ \pm}^{\prime}\left(s_{i}\right)$ denote initial $\left(s=s_{i}\right)$ values of the perturbed envelope coordinates. The \pm normal mode solutions to the homogeneous equation correspond to the well-known breathing $(+)$ and quadrupole $(-)$ modes with phase advances $\sigma_{+}$and $\sigma_{-}$[7]. Projections of the modes onto general initial envelope perturbations in $x$ and $y$ are given by Eq. (53) and the pure mode launching conditions are presented in Sec. II F and are illustrated in Fig. 6. The stability of these undriven oscillations is expected because the applied forces of the continuous focusing lattice cannot change the energy of the oscillations. Variations of the normalized phase advances $\sigma_{ \pm} / \sigma_{0}$ of the breathing and quadrupole modes calculated from Eq. (64) are plotted in Fig. 7 as a function of $\sigma / \sigma_{0}$ over the full range of space-charge intensity $0 \leq$ $\sigma / \sigma_{0} \leq 1$. As expected for finite space-charge intensity with $\sigma / \sigma_{0}<1$, the phase advance of the breathing mode is always greater than the phase advance of the quadrupole mode. Also, consistent with Eq. (56), the zero spacecharge limit phase advances satisfy $\lim _{\sigma \rightarrow \sigma_{0}} \sigma_{ \pm}=2 \sigma_{0}$. The homogeneous solutions for the normal envelope modes given in Eq. (65) are also straightforward to derive using the matrix formulation in Sec. II F. This derivationis carried out in Appendix G [29]. Results presented in Appendix $G$ provide insight on the matrix formulation and may prove useful as a conceptual guide for calculations with periodic lattices where explicit transformations to decoupled variables are not possible.

A Green's function method [19] based on the homogeneous solutions in Eq. (65) can be employed to calculate particular solutions of Eq. (63) in terms of an integral as

$$
\begin{aligned}
& \frac{\delta r_{ \pm}(s)}{r_{m}}=\frac{1}{L_{p}^{2}} \int_{s_{i}}^{s} d \tilde{s} G_{ \pm}(s, \tilde{s}) \delta p_{ \pm}(\tilde{s}) \\
& \delta p_{+}(s)=-\frac{\sigma_{0}^{2}}{2}\left[\frac{\delta \kappa_{x}(s)}{k_{\beta 0}^{2}}+\frac{\delta \kappa_{y}(s)}{k_{\beta 0}^{2}}\right]+\left(\sigma_{0}^{2}-\sigma^{2}\right) \frac{\delta Q(s)}{Q}+\sigma^{2}\left[\frac{\delta \varepsilon_{x}(s)}{\varepsilon}+\frac{\delta \varepsilon_{y}(s)}{\varepsilon}\right] \\
& \delta p_{-}(s)=-\frac{\sigma_{0}^{2}}{2}\left[\frac{\delta \kappa_{x}(s)}{k_{\beta 0}^{2}}-\frac{\delta \kappa_{y}(s)}{k_{\beta 0}^{2}}\right]+\sigma^{2}\left[\frac{\delta \varepsilon_{x}(s)}{\varepsilon}-\frac{\delta \varepsilon_{y}(s)}{\varepsilon}\right]
\end{aligned}
$$

where 


$$
G_{ \pm}(s, \tilde{s})=\frac{1}{\sigma_{ \pm} / L_{p}} \sin \left(\sigma_{ \pm} \frac{s-\tilde{s}}{L_{p}}\right)
$$

are Green's functions for the breathing $(+)$ and quadrupole $(-)$ terms. The particular solution in Eq. (66) satisfies the initial conditions $\delta r_{ \pm}\left(s=s_{i}\right)=0$ and $\delta r_{ \pm}^{\prime}\left(s=s_{i}\right)=0$, and the solution can be readily interpreted as a scaled convolution of the driving perturbations onto the natural harmonic response of the normal modes. This is expected because continuous focusing is spatially homogeneous.

Given specific initial mismatches (i.e., $\delta r_{j}$ and $\delta r_{j}^{\prime}$ at $s=s_{i}$ for $j=x, y$ ) and driving terms (i.e., $\delta \kappa_{j}, \delta Q$, and $\delta \varepsilon_{j}$ ), Eqs. (65) and (66) for the homogeneous (denoted $\left.\right|_{h}$ here) and particular (denoted $\left.\right|_{p}$ here) solution components can be applied to calculate the general evolution of the envelope perturbations as $\delta r_{x}=\left.\delta r_{x}\right|_{h}+\left.\delta r_{x}\right|_{p}=\left.\left(r_{+}+r_{-}\right)\right|_{h}+\left(r_{+}+\right.$ $\left.r_{-}\right)\left.\right|_{p}$ and $\delta r_{y}=\left.\delta r_{y}\right|_{h}+\left.\delta r_{y}\right|_{p}=\left.\left(r_{+}-r_{-}\right)\right|_{h}+\left.\left(r_{+}-r_{-}\right)\right|_{p}$. To better understand the range of possible envelope evolutions the driving terms induce, it is instructive to calculate the response to specific classes of driving perturbations that are adiabatic, sudden, ramped, and harmonic.

First, in the adiabatic limit, variations of the driving terms $\delta p_{ \pm}(s)$ are taken to be slow on the scale of the quadrupole mode wavelength $2 \pi L_{p} / \sigma_{-}$, which is longer than the breathing mode wavelength $2 \pi L_{p} / \sigma_{+}$for $\sigma / \sigma_{0}<1$. In this situation, Eqs. (63) and (64) can be employed to express the particular solutions as

$$
\begin{aligned}
& \frac{\delta r_{+}}{r_{m}}=\frac{\delta p_{+}}{\sigma_{+}^{2}}=-\left[\frac{1}{2} \frac{1}{1+\left(\sigma / \sigma_{0}\right)^{2}}\right] \frac{1}{2}\left(\frac{\delta \kappa_{x}}{k_{\beta 0}^{2}}+\frac{\delta \kappa_{y}}{k_{\beta 0}^{2}}\right)+\left[\frac{1}{2} \frac{1-\left(\sigma / \sigma_{0}\right)^{2}}{1+\left(\sigma / \sigma_{0}\right)^{2}}\right] \frac{\delta Q}{Q}+\left[\frac{\left(\sigma / \sigma_{0}\right)^{2}}{1+\left(\sigma / \sigma_{0}\right)^{2}}\right] \frac{1}{2}\left(\frac{\delta \varepsilon_{x}}{\varepsilon}+\frac{\delta \varepsilon_{y}}{\varepsilon}\right), \\
& \frac{\delta r_{-}}{r_{m}}=\frac{\delta p_{-}}{\sigma_{-}^{2}}=-\left[\frac{1}{1+3\left(\sigma / \sigma_{0}\right)^{2}}\right] \frac{1}{2}\left(\frac{\delta \kappa_{x}}{k_{\beta 0}^{2}}-\frac{\delta \kappa_{y}}{k_{\beta 0}^{2}}\right)+\left[\frac{2\left(\sigma / \sigma_{0}\right)^{2}}{1+3\left(\sigma / \sigma_{0}\right)^{2}}\right] \frac{1}{2}\left(\frac{\delta \varepsilon_{x}}{\varepsilon}-\frac{\delta \varepsilon_{y}}{\varepsilon}\right) .
\end{aligned}
$$

The adiabatic particular solutions in Eq. (68) can also be derived by partial integration of Eq. (66) and neglecting the terms containing $\delta p_{ \pm}^{\prime}$. Coefficients of the driving terms $\left(\delta \kappa_{x} / k_{\beta 0}^{2} \pm \delta \kappa_{y} / k_{\beta 0}^{2}\right) / 2, \delta Q / Q$, and $\left(\delta \varepsilon_{x} / \varepsilon \pm \delta \varepsilon_{y} / \varepsilon\right) / 2$ in the adiabatic solutions (i.e., the terms in the square brackets) are plotted as a function of space-charge depression $\sigma / \sigma_{0}$ in Fig. 8. These curves describe the relative strength of the response in $\delta r_{ \pm}$to different classes of adiabatic driving perturbations as a function of space-charge intensity.

Next, to analyze the opposite limit of driven perturbations $\delta p_{ \pm}(s)$ with sudden changes, we take

$$
\delta p_{ \pm}(s)={\widehat{\delta p_{ \pm}}} \Theta\left(s-s_{p}\right) \text {. }
$$

Here, $\Theta(x)$ is the Heaviside step function defined by $\Theta(x)=0$ for $x<0$ and $\Theta(x)=1$ for $x>0, s=s_{p}$ is the axial coordinate where the perturbations jump, and

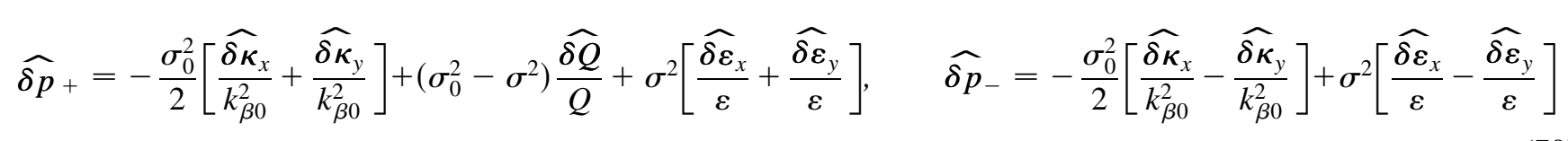

are constant amplitudes of the sudden, step perturbations defined according to Eq. (66) with $\delta Q \rightarrow \widehat{\delta Q}=$ const for the perveance perturbation, etc. For these step-function perturbations, Eq. (66) can be integrated, and the response in the particular solutions for $\delta r_{ \pm}$can be expressed in the same form as the adiabatic solutions with the following substitutions in Eq. (68):

$$
\delta p_{ \pm} \rightarrow \widehat{\delta p_{ \pm}}\left[1-\cos \left(\sigma_{ \pm} \frac{s-s_{p}}{L_{p}}\right)\right] \Theta\left(s-s_{p}\right)
$$

The response to other sudden perturbations $\delta p_{ \pm}(s)$ can be calculated by superimposing perturbations of the form in Eqs. (69) and (70).

Comparing the adiabatic solutions in Eq. (68) with the sudden solutions given by Eqs. (68) and (71), note that the sudden response is oscillatory with periods $2 \pi L_{p} / \sigma_{ \pm}$in the solutions for $\delta r_{ \pm}$rather than steady as in the adiabatic

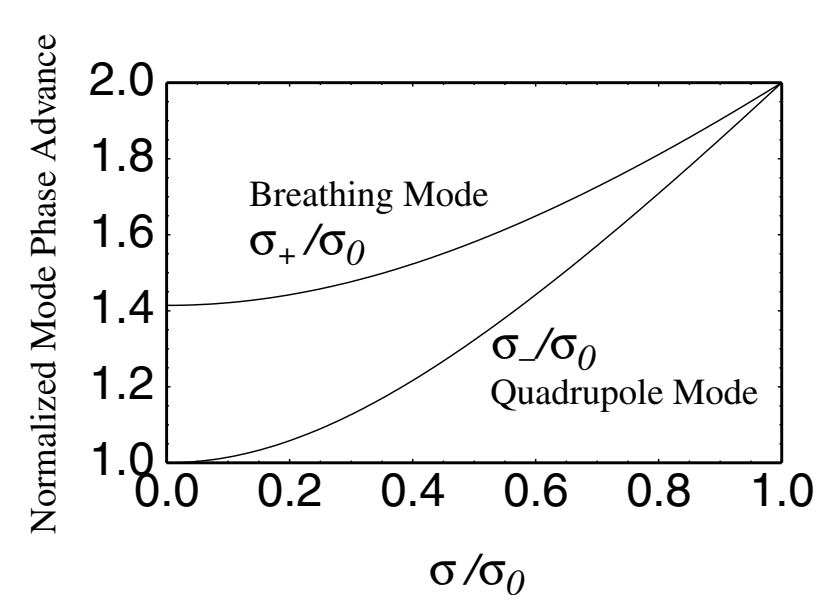

FIG. 7. Normalized phase advances $\sigma_{+} / \sigma_{0}$ and $\sigma_{-} / \sigma_{0}$ of the breathing and quadrupole modes plotted versus $\sigma / \sigma_{0}$ for a continuous focusing channel. 
a) $\delta r_{+}=\left(\delta r_{x}+\delta r_{y}\right) / 2$

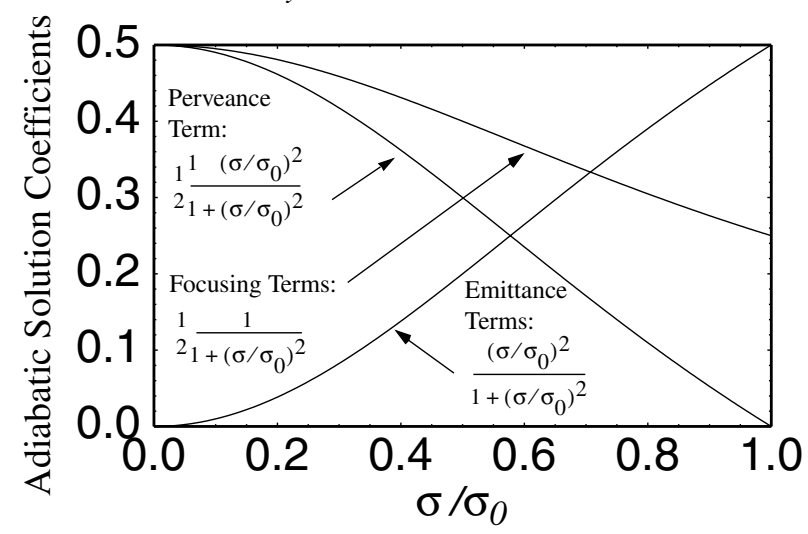

b) $\delta r_{-}=\left(\delta r_{x}-\delta r_{y}\right) / 2$

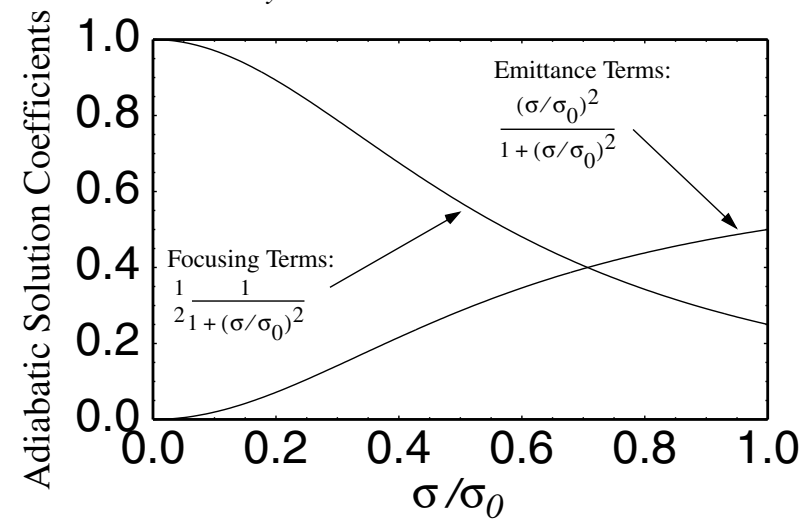

FIG. 8. Coefficients in Eq. (68) describing the strength of adiabatic limit envelope response terms to classes of driving perturbations are plotted as a function of $\sigma / \sigma_{0}$. Coefficients occurring in the sum $\left(r_{+}\right)$and difference $\left(r_{-}\right)$particular solutions are plotted separately in (a) and (b).

case. For the same driving perturbation amplitude [e.g., $\delta Q(s)=\widehat{\delta Q}$ for $s \gtrsim s_{p}$ for the adiabatic perturbation], the excursions of the envelope oscillations for sudden perturbations oscillate between zero and twice the adiabatic excursion. This characteristic response to a stepfunction perturbation is illustrated in Fig. 9 for the evolution of $\delta r_{+} / r_{m}$ resulting from a pure emittance perturbation with $\left(\widehat{\delta \varepsilon}_{x} / \varepsilon+\widehat{\delta \varepsilon}_{y} / \varepsilon\right) / 2>0$. The dashed line shows the adiabatic response for a slowly varied perturbation that achieves the same emittance change as the sudden perturbation applied at $s=s_{p}$ [i.e., an adiabatic perturbation with $\left[\delta \varepsilon_{x}(s) / \varepsilon+\delta \varepsilon_{y}(s) / \varepsilon\right] / 2=$ $\left(\widehat{\delta \varepsilon_{x}} / \varepsilon+\widehat{\delta \varepsilon_{y}} / \varepsilon\right) / 2$ for $\left.s \gtrsim s_{p}\right]$.

Another instructive example is the envelope response to linear ramp driving perturbations. In this case we take

$$
\delta p_{ \pm}(s)={\widehat{\delta p_{ \pm}}}_{ \pm}^{\prime}\left(s-s_{p}\right) \Theta\left(s-s_{p}\right) .
$$

Here, $\widehat{\delta p}_{ \pm}^{\prime}$ are constant gradients that describe the ramp perturbations applied at axial coordinate $s=s_{p}$ and are defined by replacing $\widehat{\delta Q} \rightarrow \widehat{\delta Q^{\prime}}$ with $\widehat{\delta Q}^{\prime}=$ const in Eq. (70) for the perveance perturbation, etc. For these

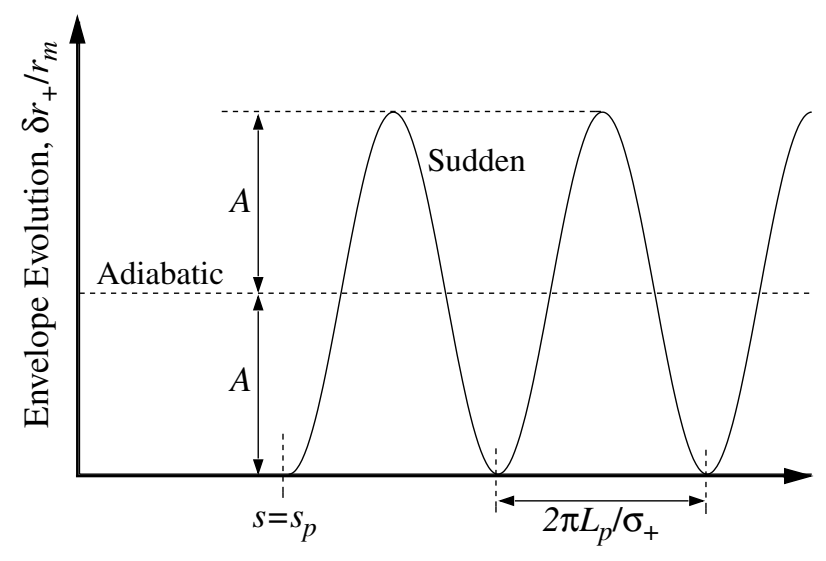

Axial Coordinate, $s$

FIG. 9. Contrast of the envelope response $\delta r_{+}(s)$ to sudden and adiabatic driving emittance perturbations with $\left(\widehat{\delta}_{x} / \varepsilon+\right.$ $\left.\widehat{\delta}_{y} / \varepsilon\right) / 2>0$ that achieve the same value of emittance change for $s>s_{p}$.

ramp perturbations, the particular solutions given by Eq. (66) can be expressed in the same form as the adiabatic solutions with the following substitutions in Eq. (68):

$$
\begin{aligned}
\delta p_{ \pm} \rightarrow \frac{L_{p}}{\sigma_{ \pm}} \widehat{\delta p}_{ \pm}^{\prime}[ & \sigma_{ \pm} \frac{s-s_{p}}{L_{p}} \\
& \left.+\sin \left(\sigma_{ \pm} \frac{s-s_{p}}{L_{p}}\right)\right] \Theta\left(s-s_{p}\right) .
\end{aligned}
$$

Comparing the solutions for adiabatic, sudden, and linear ramped driving perturbations given by Eqs. (68)-(73), the response to the ramped perturbations can be interpreted as having both adiabatic and "sudden" oscillatory components given by the first and second terms in Eq. (73), respectively. The particular solution for the ramped perturbation is illustrated schematically in Fig. 10 for the evolution of $\delta r_{+} / r_{m}$ in response to $\widehat{a}$, pure emittance perturbation with $\left(\widehat{\delta}_{x}^{\prime} / \varepsilon+\right.$ $\left.\widehat{\delta \varepsilon}_{y}^{\prime} / \varepsilon\right) / 2>0$. For $s \gg s_{p}$, the response is dominated by the adiabatic component, whereas for $s \gtrsim s_{p}$, the sudden oscillatory component with period $2 \pi L_{p} / \sigma_{+}$ can be significant.

Finally, we analyze harmonic driving perturbations in $\delta p_{ \pm}(s)$ by taking

$$
\delta p_{ \pm}(s)=\widehat{\delta p}_{ \pm} \exp (i k s)
$$

Here, $\widehat{\delta p}_{+}$are complex constants defined from $\delta p_{ \pm}(s)$ that are of the same form as given in Eq. (70) and represent the amplitude of the harmonic perturbations, and $k=$ const is the complex wave number of the perturbations. The physical perturbations are represented by the real part of the expressions in Eq. (74). Driving perturbations with pure harmonic oscillations can be modeled by taking $k$ real, pure exponential growing or damping by 
taking $k$ imaginary, and damped or growing oscillations by taking $k$ complex. Superpositions of harmonic perturbations of the form in Eq. (74) can be employed to recover the previous examples analyzed. For these harmonic per- turbations, the particular solutions given by Eq. (66) can be expressed in the same form as the adiabatic solution by taking the real part of the following substitutions in Eq. (68):

$$
\delta p_{ \pm} \rightarrow \widehat{\delta p_{ \pm}}\left[\frac{\cos \left(\sigma_{ \pm} s / L_{p}\right)+i\left(k L_{p} / \sigma_{ \pm}\right) \sin \left(\sigma_{ \pm} s / L_{p}\right)-\exp (i k s)}{\left(k L_{p} / \sigma_{ \pm}\right)^{2}-1}\right]
$$

For $k$ pure imaginary the particular solutions given by Eqs. (68) and (75) lead to oscillatory solutions with damping $(\operatorname{Im} k>0)$ or growth terms $(\operatorname{Im} k<0)$. For $|\operatorname{Re} k|$ sufficiently removed from $\sigma_{ \pm} / L_{p}$, the response is pure oscillatory ( $\operatorname{Im} k=0)$, or oscillatory with growth or damping terms $(\operatorname{Im} k \neq 0)$. When $|\operatorname{Re} k| \simeq \sigma_{ \pm} / L_{p}$, the response has classic linear resonance growth with

$$
\lim _{k \rightarrow \sigma_{ \pm} / L_{p}} \frac{\cos \left(\sigma_{ \pm} s / L_{p}\right)+i\left(k L_{p} / \sigma_{ \pm}\right) \sin \left(\sigma_{ \pm} s / L_{p}\right)-\exp (i k s)}{\left(k L_{p} / \sigma_{ \pm}\right)^{2}-1}=\frac{i}{2}\left[\sin \left(\sigma_{ \pm} s / L_{p}\right)-\left(\sigma_{ \pm} s / L_{p}\right) \exp \left(\sigma_{ \pm} s / L_{p}\right)\right]
$$

Linear superposition of the specific examples above provides insight into the range of envelope responses to small-amplitude driving perturbations formally given by Eq. (66). Several conclusions can be drawn from these results. First, driving perturbations that are fast on the scale of the breathing and quadrupole envelope mode phase advances $\sigma_{ \pm}$are more dangerous than slow changes because they can result in twice the beam envelope excursion for equal amplitude changes in the driving terms. Second, oscillatory driving perturbations of small amplitude are not problematic unless the driving terms have frequency components with phase advances close to the breathing or quadrupole mode phase advances $\sigma_{ \pm}$. This simple continuous focusing calculation of driven perturbations should help guide analysis of the more complicated situation for driving perturbations in periodic focusing lattices. However, caution must be employed in the application of these results, particularly when making higher-order extrapolations. For example, if a periodic focusing perturbation with $\delta \kappa_{x}=$ $-\delta \kappa_{y}$ is taken, one might expect to recover net focusing

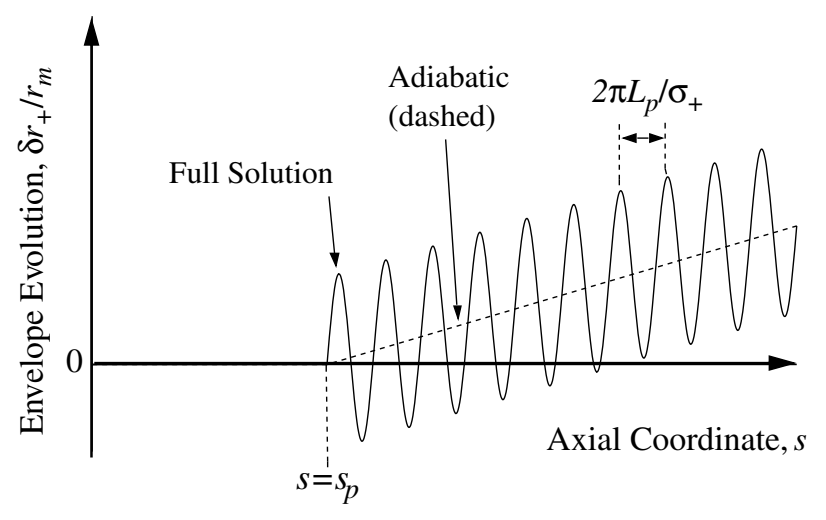

FIG. 10. Envelope response $\delta r_{+}(s)$ for a linear ramp driving emittance perturbation with $\left(\widehat{\delta \varepsilon}_{x}^{\prime} / \varepsilon+\widehat{\delta \varepsilon}_{y}^{\prime} / \varepsilon\right) / 2>0$. because this perturbation corresponds to a superimposed alternating gradient quadrupole lattice. However, from Eq. (66), such a choice has no projection on the breathing mode to linear order. A recent analytical study of matched beam envelopes in periodic quadrupole focusing channels suggests how such higher-order effects might be more accurately modeled but such methods are beyond the scope of the present analysis [25].

\section{ENVELOPE MODES IN SOLENOIDAL FOCUSING CHANNELS}

A beam with an elliptical cross-section envelope and zero total canonical angular momentum (i.e., $P_{\theta}=$ $\left.\left\langle x y^{\prime}-y x^{\prime}\right\rangle_{\perp}+\left[q B_{z} /\left(2 m \gamma_{b} \beta_{b} c\right)\right]\left\langle x^{2}+y^{2}\right\rangle_{\perp}=0\right)$ that is focused in a solenoidal transport channel by a periodic solenoidal magnetic field $\mathbf{B}(s)=-\left[B_{z}^{\prime}(s) / 2\right](x \hat{\mathbf{x}}+y \hat{\mathbf{y}})+$ $B_{z}(s) \hat{\mathbf{z}}$ can be analyzed with the envelope equation (4), provided that we take the focusing strength to be $\kappa_{x}=$ $\kappa_{y} \equiv \kappa=\left[q B_{z} /\left(2 m \gamma_{b} \beta_{b} c\right)\right]^{2}$ and interpret the analysis as applying in a local ( $s$-varying) rotating Larmor frame. This correspondence is developed in Appendix A by deriving a self-consistent KV distribution describing elliptical beams with $P_{\theta}=0$ in a solenoidal focusing channel. The derivation identifies correspondences needed to apply Eq. (4) to this situation including replacements for the laboratory-frame rms emittances $\varepsilon_{x}$ and $\varepsilon_{y}$ in terms of Larmor-frame invariant emittance measures. In the context of interpreting the envelope equation (4) with appropriate Larmor-frame replacements, the formalism in Sec. II F can be applied to analyze elliptical perturbations in the beam envelope for solenoidal transport channels. For simplicity of presentation, we carry out the analysis in this section without making explicit reference to the Larmor frame. However, the Larmorframe transformations presented in Appendix A must be applied to project nonaxisymmetric normal modes $\left(\delta r_{x} \neq \delta r_{y}\right)$ into the stationary laboratory frame to 
view the evolution there - a distinction not made in previous work on envelope modes in solenoidal transport channels $[2,7]$.

In this section we explore normal mode envelope perturbations $(\delta \mathbf{P}=0)$ in a periodic solenoidal transport channel with piecewise constant $\kappa(s)$. Physical solenoidal focusing lattices with fringe fields associated with continuously varying $\kappa(s)$ can be approximated by the model with piecewise constant $\kappa(s)$ using the equivalency procedures developed in Appendix C. Mode structures and launching conditions are classified and instabilities are parametrically mapped using the decoupled mode formulation in Sec. II F. In addition to elliptical beam perturbations with zero beam canonical angular momentum $\left(P_{\theta}=0\right)$, results for axisymmetric perturbations $\left(\delta r_{x}=\right.$ $\left.\delta r_{y}\right)$ can also be applied to beams with nonzero canonical angular momentum using the correspondences derived in Appendix A. Results in this section can also be applied without transformation to idealized beam models where a stationary, partially neutralizing charge distribution produces the specified focusing function $\kappa(s)$. Driving perturbations with $\delta \mathbf{P} \neq 0$ are not analyzed.

\section{A. Matched envelope solution}

We consider the case of a periodic, matched solution $\left(r_{x}=r_{x m}\right.$ and $\left.r_{y}=r_{y m}\right)$ to the envelope equation (4) with equal emittances $\varepsilon=\varepsilon_{x}=\varepsilon_{y}$ and a round beam envelope $r_{x m}=r_{y m}=r_{m}$. A hard-edge focusing lattice is taken with $\kappa_{x}(s)=\kappa_{y}(s) \equiv \kappa(s)$ piecewise constant as illustrated in Fig. 2(b) with lattice period $L_{p}$, solenoid strength $\hat{\kappa}=$ const, and occupancy $\eta$. The matched envelope solutions are parametrized by $L_{p}, \sigma_{0}, \sigma / \sigma_{0}$, and $\eta$. Numerical solutions are plotted for one lattice period in Fig. 11 in terms of the scaled envelope radius $\left[r_{m} /\left(\sqrt{2 Q} L_{p}\right)\right]$ versus normalized axial coordinate $\left(s / L_{p}\right)$ for fixed undepressed single-particle phase advance $\sigma_{0}$, and values of $\sigma / \sigma_{0}$. Solutions for large and small occupancies are contrasted in 11(a) and 11(b) and symmetry points are indicated on the plot. Comparing 11(a) and 11(b), observe that the radial extent of the periodic flutter of the beam envelope varies strongly with the solenoid occupancy $\eta$. This strong flutter variation is expected because the limit $\eta \rightarrow 1$ corresponds to continuous focusing in the Larmor frame with $r_{m}=$ const, whereas the limit $\eta \rightarrow 0$ corresponds to focusing with thin-lens kicks (see Appendix H) and maximal flutter motion. The single-particle phase advances $\sigma_{0}$ and $\sigma$ are interpreted in the Larmor frame.

The analysis in Appendix A shows that the matched, round beam envelope with $r_{x m}=r_{y m}=r_{m}$ described above can be applied to model beams with finite canonical angular momentum $P_{\theta}=\left\langle x y^{\prime}-y x^{\prime}\right\rangle_{\perp}+$ $\left[q B_{z} /\left(2 m \gamma_{b} \beta_{b} c\right)\right]\left\langle x^{2}+y^{2}\right\rangle_{\perp}$ in the range $-1<2 P_{\theta} / \varepsilon<$ 1 if the emittance $\varepsilon$ is replaced by the Larmor invariant emittance according to $\varepsilon^{2} \rightarrow \varepsilon_{x}^{2}-4\left\langle r^{2} \theta^{\prime}\right\rangle_{\perp}^{2}+4 P_{\theta}^{2}=$ a) $\sigma_{0}=80^{\circ}$ and $\eta=0.75$

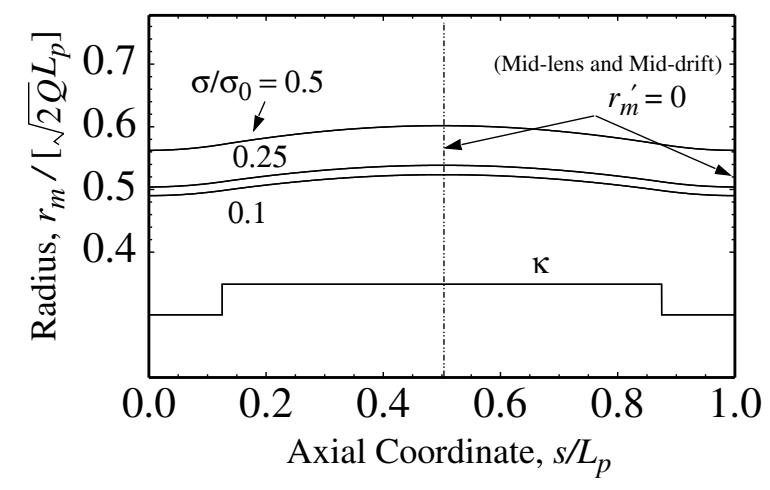

b) $\sigma_{0}=80^{\circ}$ and $\eta=0.25$

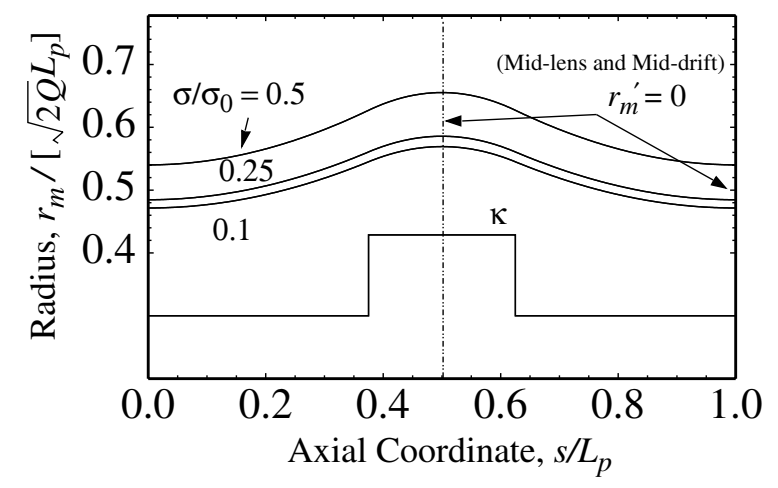

FIG. 11. Scaled matched beam envelope $r_{m} /\left(\sqrt{2 Q} L_{p}\right)$ versus normalized axial coordinate $s / L_{p}$ for one period of a hardedge solenoidal focusing channel in Fig. 2(b). Solutions are shown for $\sigma_{0}=80^{\circ}$ and values of $\sigma / \sigma_{0}$ indicated for occupancies $\eta=0.75$ and $\eta=0.25$ in (a) and (b), respectively.

const to include the defocusing effect of $P_{\theta} \neq 0$. The matched beam parametrization employed with $L_{p}, \sigma_{0}$, $\sigma / \sigma_{0}$, and $\eta$ is convenient to understand envelope stability properties in solenoidal focusing and places results in a form directly comparable to those obtained for quadrupole focusing (see Sec. V). However, in calculating perveance (or current) limits, the periodicity of the lattice plays a less direct role for solenoidal focusing than for quadrupole focusing [23,25]. For a quadrupole lattice alternating focusing and defocusing optics in the period provide net focusing that cannot exist without the regular interchange of focusing and defocusing within the period, whereas in solenoid lattices all optics are focusing and net focusing exists even in the limit of full occupancy $(\eta=1)$ where there is no lattice period.

\section{B. Normal modes}

Normal mode solutions for elliptical perturbations $\left(\delta r_{x} \neq \delta r_{y}\right)$ about the round, matched envelope solutions described in Sec. IVA are numerically analyzed using the formulation in Sec. II F. For the special case considered of an axisymmetric matched envelope the reduced 
eigenvalue formulation with decoupled perturbed envelopes $\delta r_{ \pm}=\left(\delta r_{x} \pm \delta r_{y}\right) / 2$ is employed to resolve the perturbations into breathing $(+)$ and quadrupole $(-)$ modes. This reduced problem is equivalent to numerically calculating eigenvalues $\lambda_{n}$ of the coupled four-dimensional matrix equation (47) to characterize the stability properties of the homogeneous equation (38) with $\kappa_{x}=$ $\kappa_{y}=\kappa, \varepsilon_{x}=\varepsilon_{y}=\varepsilon$, and zero driving perturbations $(\delta \mathbf{P}=0)$. Eigenvalues are calculated for specified values of $\eta$ and ranges of $\sigma_{0}$ and $\sigma / \sigma_{0}$ to map out mode properties including mode phase advances $\sigma_{ \pm}$and growth factors $\gamma_{ \pm}$associated with broad bands of parametric instability. Results we obtained are displayed in Figs. 12-14.

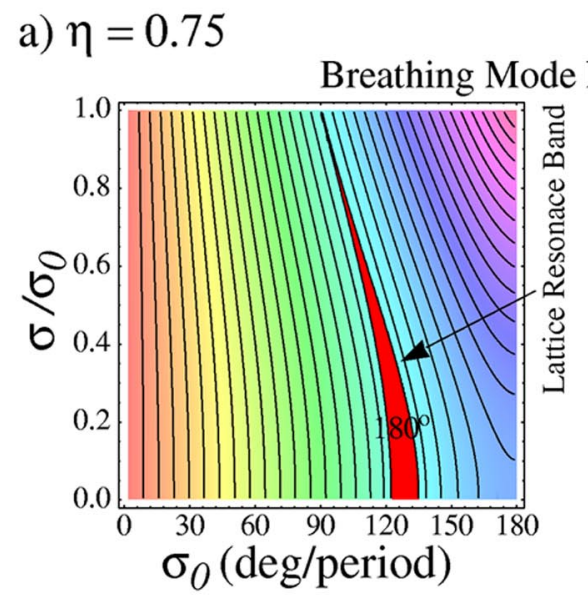

b) $\eta=0.25$

Phase Advance, $\sigma_{+}$

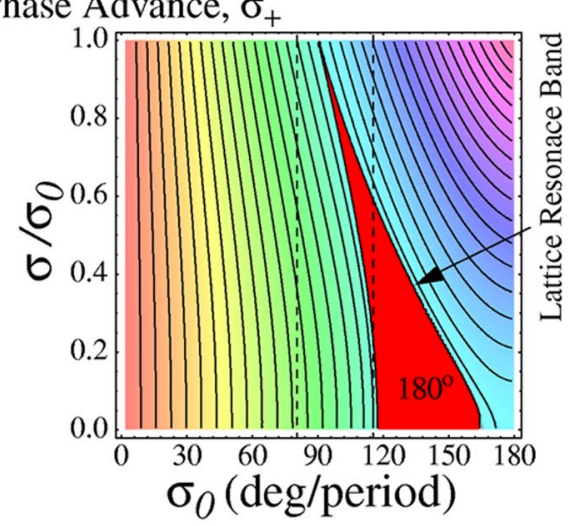

Quadrupole Mode Phase Advance, $\sigma_{-}$
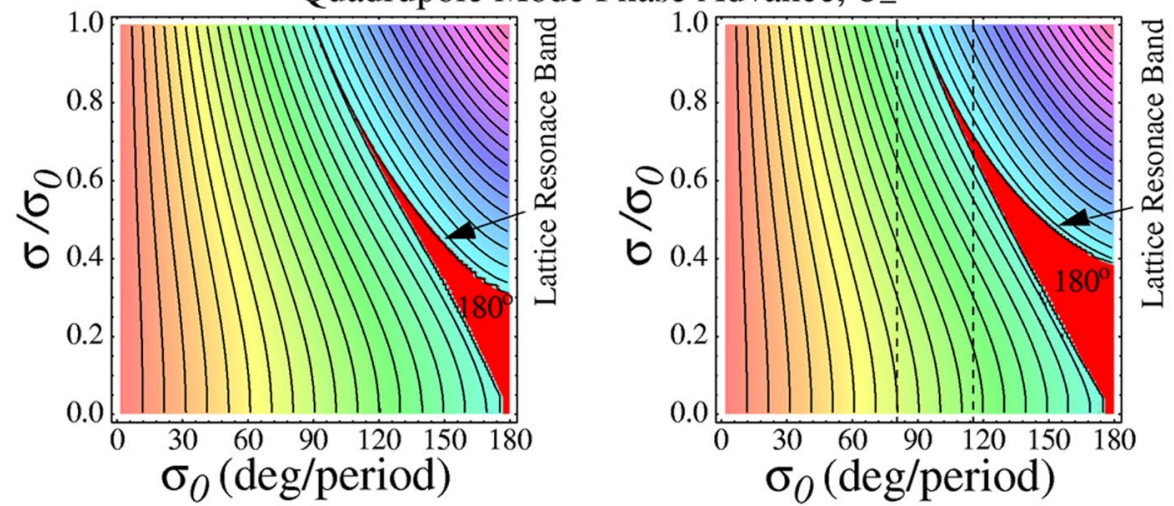

Breathing and Quadrupole Mode Growth Factors, $\gamma_{+}$and $\gamma_{-}$
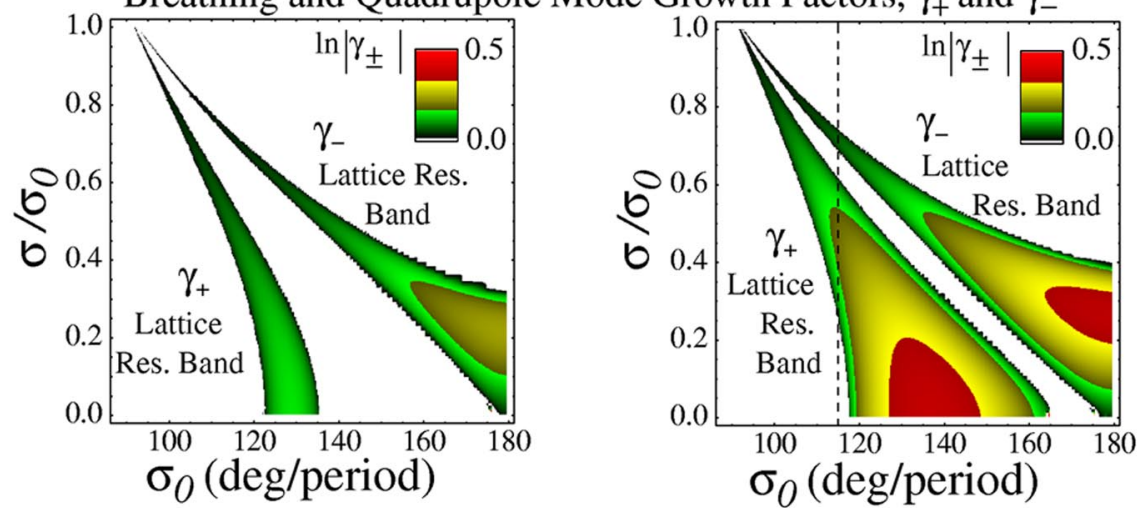

FIG. 12. (Color) Contours of (upper row) breathing and (middle row) quadrupole mode phase advances $\left(\sigma_{ \pm}\right)$and (bottom row) growth factors $\left(\gamma_{ \pm}\right)$as a function of $\sigma_{0}$ and $\sigma / \sigma_{0}$ for a solenoidal focusing channel. Results are shown for occupancies $\eta=0.75$ and $\eta=0.25$ in columns (a) and (b). In the upper and middle rows, black contour lines correspond to $\sigma_{ \pm}=10^{\circ}, 20^{\circ}, 30^{\circ}, \ldots$, and colors vary continuously with changes in $\sigma_{ \pm}$(bands of instability with $\sigma_{ \pm}=180^{\circ}$ are marked in solid red for emphasis). In the lower row, values of $\ln \left(\gamma_{ \pm}\right)$with $\gamma_{ \pm}>1$ are labeled with continuously varying colors as indicated in the key. Identical color schemes are used in (a) and (b) to measure mode phase advances and growth factors. Vertical dashed lines in the plots for $\eta=0.25$ correspond to the cross-section plots in Fig. 13. 

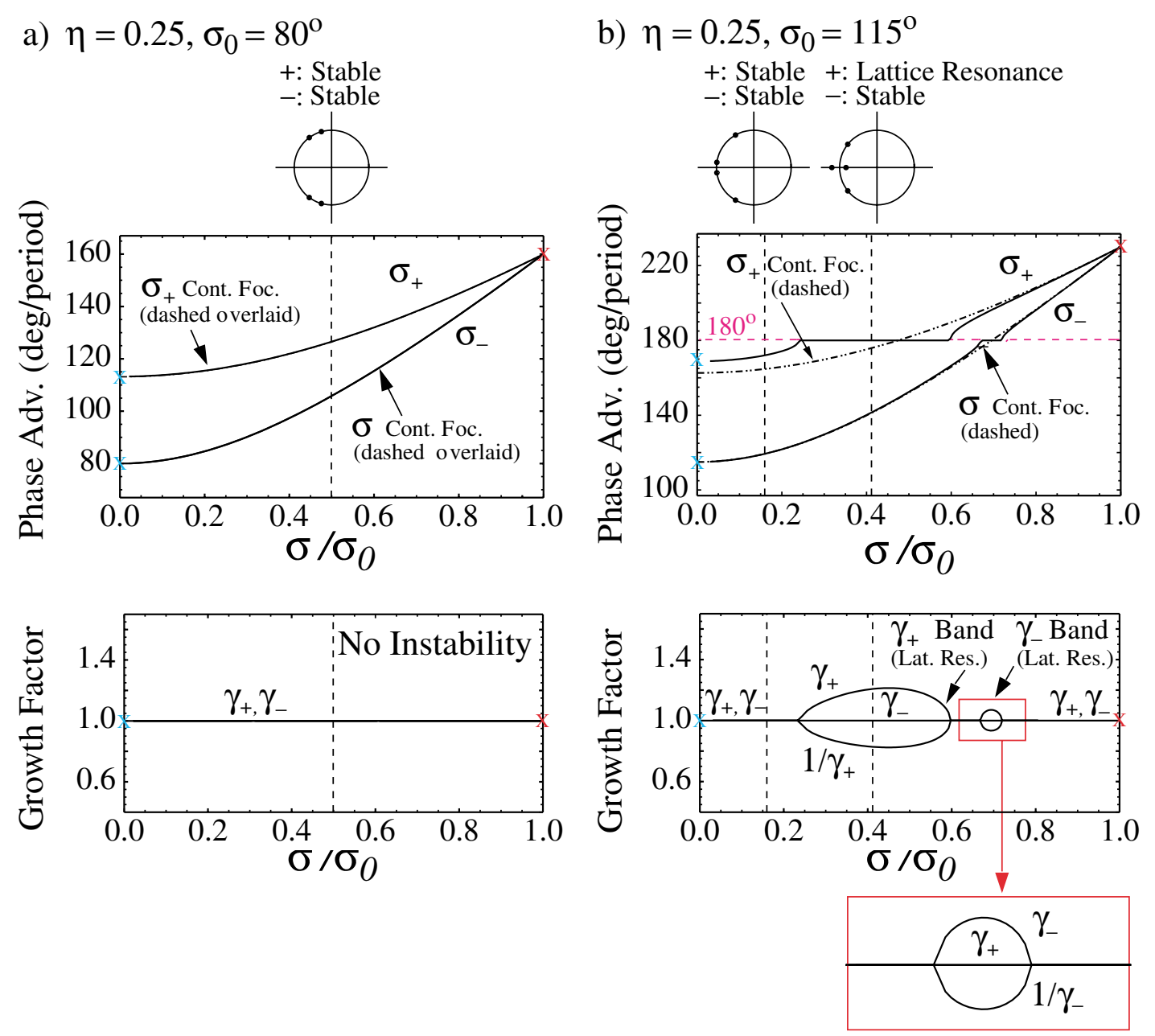

FIG. 13. (Color) Phase advance $\left(\sigma_{ \pm}\right)$and growth factors $\left(\gamma_{ \pm}\right)$of the breathing and quadrupole envelope modes versus $\sigma / \sigma_{0}$ for a solenoidal focusing channel with occupancy $\eta=0.25$ for $\sigma_{0}=80^{\circ}$ and $\sigma_{0}=115^{\circ}$ in (a) and (b). Continuous focusing model predictions for $\sigma_{ \pm}$are superimposed (dashed curves). Red $\times \mathrm{s}$ mark analytical results in the zero space-charge limit and cyan $\times \mathrm{s}$ mark thin-lens limit $(\eta \rightarrow 0)$ analytical results with $\sigma / \sigma_{0}=0$. Vertical dashed lines correspond to locations of plots of complex eigenvalues $\lambda_{ \pm}$placed above. Red boxes denote expanded views.

We find that bands of parametric instability with $\gamma_{+}>$ 1 and $\gamma_{-}>1$ for the breathing and quadrupole modes do not overlap. Therefore, in terms of the symmetry classes presented in Fig. 4, eigenvalues can only fall into class (a) (stability) or (c) (lattice resonance instability), and class (d) (double lattice resonance instability) does not occur. As shown in Sec. II F, eigenvalues falling into class (b) (confluent resonance instability) are not possible because the breathing and quadrupole modes are decoupled for solenoidal focusing. Breathing and quadrupole mode phase advances $\sigma_{ \pm}$and growth factors $\gamma_{ \pm}$are contoured in Fig. 12 as a function of $\sigma_{0}$ and $\sigma / \sigma_{0}$ for $\sigma_{0} \in\left(0,180^{\circ}\right)$ and the full range of space-charge depression $\sigma / \sigma_{0} \in$ $(0,1]$. Results are contrasted for occupancies $\eta=0.75$ and $\eta=0.25$ in Figs. 12(a) and 12(b). Growth factors of both the breathing $\left(\gamma_{+}>1\right)$ and quadrupole $\left(\gamma_{-}>1\right)$ modes are superimposed on the same plots because the bands do not overlap, and growth factors of the corresponding damped modes are not plotted because damping factors are given by $1 / \gamma_{ \pm}$of the growing mode [see Fig. 4(c) and Table I]. The growth factor contours are restricted to $\sigma_{0} \in\left(90^{\circ}, 180^{\circ}\right)$ because no instability is found for $\sigma_{0}<90^{\circ}$. These plots help clarify regions of parametric envelope instability observed in previous studies $[4,7,26]$. In the present study, modes are unambiguously identified over the practical range of machine parameters and errors in branch calculations presented in previous studies are corrected.

To better illustrate the mode structures presented in Fig. 12, in Fig. 13 we plot the mode phase advances and growth factors for fixed $\sigma_{0}$ and $\eta$ as a function of $\sigma / \sigma_{0}$. Curves shown correspond to the vertical dashed lines on the contour plots in Fig. 12. Complex plots of the eigenvalues $\lambda_{ \pm}$relative to the unit circle are superimposed at several locations to further illustrate the eigenvalue structure of the modes and the symmetry class of the 
instability bands. Individual mode branches for the breathing and quadrupole modes are identified outside of regions of instability using the formulation described in Sec. II F and branches are continuously connected across bands of parametric instability. As expected for finite space charge $\left(\sigma / \sigma_{0}<1\right)$, the in-phase oscillations of the breathing mode with $\delta r_{x}=\delta r_{y}$ have higher phase advance $\sigma_{+}$than the phase advance $\sigma_{-}$of the out-ofphase oscillations of the quadrupole mode with $\delta r_{x}=$ $-\delta r_{y}$. Continuous model results superimposed are calculated from Eq. (64) using the single-particle phase advances $\sigma_{0}$ and $\sigma$ derived from the periodically focused, matched beam. Far from instability bands, the continuous formulas provide accurate estimates of the mode phase advances [7]. Curves for the continuous and periodic focused calculations of $\sigma_{ \pm}$almost overlay for $\sigma_{0}=80^{\circ}$ and agreement further improves for lower values of $\sigma_{0}$. Analysis in Sec. II F [see Eq. (56)] shows that the zero space-charge limit $Q \rightarrow 0$ corresponds to stable breathing and quadrupole mode oscillations with

$$
\lim _{\sigma \rightarrow \sigma_{0}} \sigma_{ \pm}=2 \sigma_{0}, \quad \lim _{\sigma \rightarrow \sigma_{0}} \gamma_{ \pm}=1
$$

These limiting values are indicated on the figures and provide a partial check of results. For another check of results, mode eigenvalues are analytically calculated in Appendix $\mathrm{H}$ in the thin-lens limit $\left(\eta \rightarrow 0\right.$ at fixed $\left.\sigma_{0}\right)$ for a beam with full space-charge depression $(\sigma=0)$. Phase advances and growth rates calculated from this analysis are indicated in Fig. 13(b). The small discrepancies evident are traceable to sensitivity of results to the value of solenoid occupancy $\eta$.

To better understand sensitivities on the solenoid occupancy, variations of the breathing and quadrupole mode phase advances and growth factors with $\eta$ are illustrated in Fig. 14. From this plot and Fig. 12, note that instability bands get wider and mode growth rates stronger with decreasing $\eta$. Limit points indicated on the plots come from analytical calculations of the band structure in the thin-lens limit $(\eta \rightarrow 0)$ at full space-charge depression presented in Appendix H and Eq. (77).

The lattice resonance instability bands have been identified as a half-integer parametric resonance between the envelope mode oscillation frequencies and the lattice [7]. This suggests that the location of the envelope bands can be crudely estimated using resonance conditions based on the breathing and quadrupole mode frequencies $\sigma_{ \pm}$derived from the continuous model with $\sigma_{+}=$ $\sqrt{2 \sigma_{0}^{2}+2 \sigma^{2}}$ and $\sigma_{-}=\sqrt{\sigma_{0}^{2}+3 \sigma^{2}}$ [see Eq. (64)] as $\sigma_{ \pm}=\pi$, or equivalently with $\sigma_{0}$ and $\sigma$ measured in degrees,

$$
\sqrt{2 \sigma_{0}^{2}+2 \sigma^{2}}=180^{\circ}
$$

for the breathing mode, and
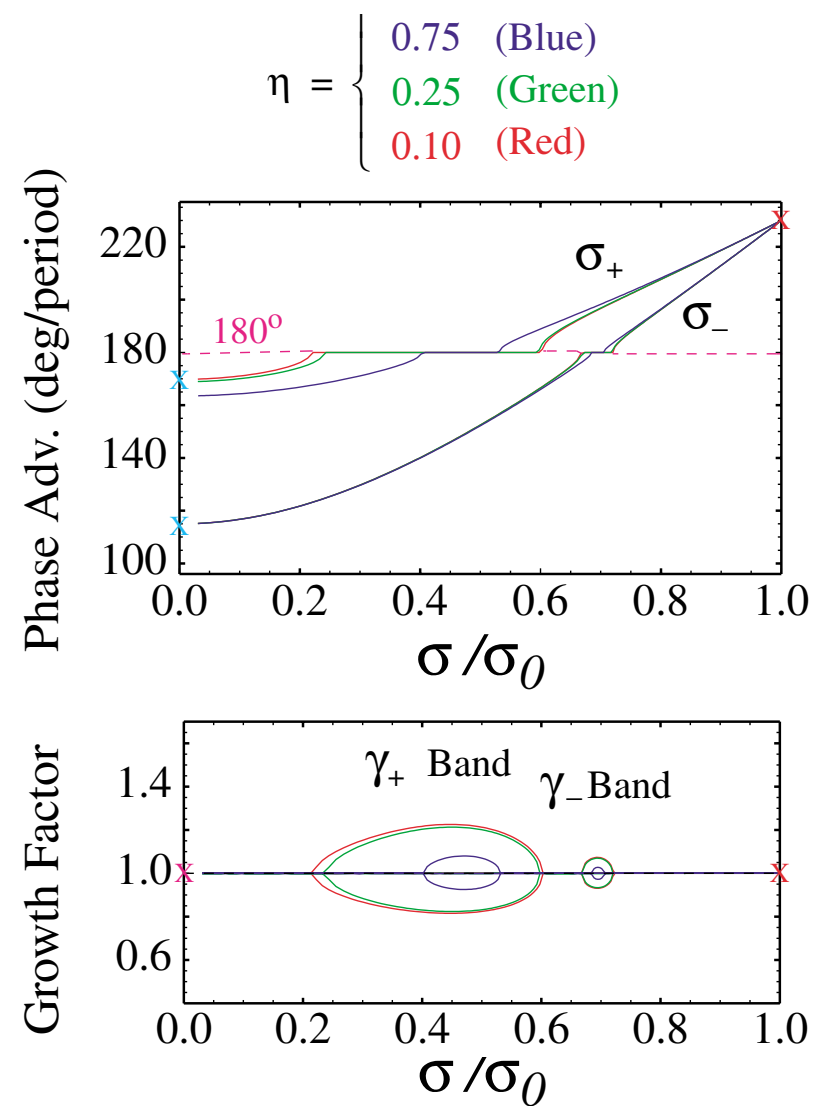

FIG. 14. (Color) Variation of mode structure with solenoid occupancy $\eta$. Phase advance $\left(\sigma_{ \pm}\right)$and growth factors $\left(\gamma_{ \pm}\right)$ of the breathing and quadrupole modes are plotted versus $\sigma / \sigma_{0}$ for $\sigma_{0}=115^{\circ}$ and the indicated values of $\eta$. Red $\times \mathrm{s}$ mark analytical results in the zero space-charge limit and cyan $\times$ s mark thin-lens limit $(\eta \rightarrow 0)$ analytical results with $\sigma / \sigma_{0}=0$.

$$
\sqrt{\sigma_{0}^{2}+3 \sigma^{2}}=180^{\circ}
$$

for the quadrupole mode. Comparing to Fig. 12, these resonance formulas roughly predict band locations, but correspond more closely to the lower thresholds in $\sigma_{0}$ than the maximum growth factor locations as might be anticipated. Moreover, the formulas cannot capture the broad parametric nature of the instability or changes in band structure with solenoid occupancy $\eta$. In order to provide better guidance for the band locations in practical applications, parametric data were employed to calculate curve fits for the instability thresholds. We find that instability threshold data can be roughly fit by curves in $\sigma_{0}$ and $\sigma$ corresponding to centered elliptical curves with

$$
\sigma^{2}+f \sigma_{0}^{2}=\left(90^{\circ}\right)^{2}(1+f)
$$

for the left- and right-boundary curves of the breathing mode band, a curve with the form 


$$
\sigma / \sigma_{0}+g \frac{\sigma_{0}}{90^{\circ}}=1+g
$$

for the left boundary of the quadrupole mode band, and a line with

$$
\sigma+g \sigma_{0}=90^{\circ}(1+g)
$$

for the right boundary of the quadrupole mode band. In Eqs. (80)-(82), $\sigma_{0}$ and $\sigma$ are measured in degrees, and $f$ and $g$ are undetermined functions of $\eta$. Based on the analysis in Sec. II F, the boundary curves in Eqs. (80)(82) are constrained to contain the limit point $\sigma=\sigma_{0}=$ $90^{\circ}$. Consistent with containing this point, Eq. (81) corresponds to the most general centered ellipse in $\sigma_{0}$ and $\sigma$, and Eqs. (81) and (82) are the most general lines in $\sigma_{0}$ and $\sigma / \sigma_{0}$, and $\sigma_{0}$ and $\sigma$, respectively. To account for weak variations from these forms in $\sigma_{0}$ and $\sigma$ while containing the point $\sigma=\sigma_{0}=90^{\circ}$, we also take $f$ and $g$ to vary linearly with $\sigma_{0}$ in addition to having dependence on $\eta$. Using threshold data with $\sigma_{0} \in\left[96^{\circ}, 174^{\circ}\right]$ and $\eta \in$ $[0.1,0.7]$, we find that the breathing mode band thresholds are fit by the elliptic formula in Eq. (80) with

$f\left(\eta, \sigma_{0}\right)= \begin{cases}1.113-0.413 \eta+0.00348 \sigma_{0}, & \text { left edge, } \\ 1.046+0.318 \eta+0.00410 \sigma_{0}, & \text { right edge, }\end{cases}$

where average and maximum deviations between the threshold data and the fit formula are $\sim 3^{\circ}$ and $\sim 6^{\circ}$ for the left edge and $\sim 4^{\circ}$ and $\sim 6^{\circ}$ for the right edge. Similarly, using the threshold data over the same range for the quadrupole mode band with the formulas in Eqs. (81) and (82), we obtain the fits

$$
g(\eta)= \begin{cases}1, & \text { left edge, } \\ 0.227+0.173 \eta, & \text { right edge, }\end{cases}
$$

where average and maximum deviations between the threshold data and the fit formulas are $\sim 5^{\circ}$ and $\sim 8^{\circ}$ for the left edge and $\sim 2^{\circ}$ and $\sim 3^{\circ}$ for the right edge. Here, we have neglected weak $\eta$ and $\sigma_{0}$ variation in the formula for the left edge of the band and we have neglected variation in $\sigma_{0}$ on the right edge to produce formulas of similar accuracy to the breathing mode band. Methods employed by Lee and Briggs [23] to analytically estimate breathing mode bandwidths for a focusing function $\kappa(s)$ with a constant and sinusoidally varying terms may be generalizable using Fourier expansions of both the piecewise constant lattice focusing function $\kappa(s)$ employed here and the matched envelope to calculate thresholds of the decoupled breathing and quadrupole bands. Future research on this topic is desirable to obtain more reliable band threshold estimates and to better understand the structure of the envelope instabilities.

As discussed in Sec. II F, the breathing and quadrupole modes are decoupled, and consequently pure modes can be simply launched at axial coordinate $s=s_{i}$ by taking initial conditions as given in Eqs. (53) and (54). This launching condition is illustrated in Fig. 6 and corresponds to cases (a) and (c) in Table I for stability and instability, respectively. For the case of instability, Table I and Figs. 4 and 5 show that certain subsets of initial conditions with either breathing or quadrupole symmetry can project onto pure exponentially damping or growing modes. A mode phase-space invariant (see Sec. II F and Appendix F) can be employed analogously to CourantSnyder invariants in single-particle dynamics to efficiently launch stable breathing or quadrupole modes with specific amplitudes and phases and calculate maximum mismatch excursions at any location in the lattice period.

As a practical matter, note in the parametric plots of the bands of mode instability presented in Fig. 12 that the breathing mode band is encountered before the quadrupole band when approaching from lower values of $\sigma_{0}$. Therefore the breathing mode instability is generally of greater importance when selecting machine operating points. Finally, it should be stressed that the breathing mode results presented can be interpreted directly as being in the laboratory frame, but the quadrupole mode rotates according to the local Larmor-frame transformations presented in Appendix A. Breathing mode stability results presented are also valid for beams with finite canonical angular momentum $P_{\theta} \neq 0$, whereas quadrupole mode results are valid only for $P_{\theta}=0$.

\section{ENVELOPE MODES IN QUADRUPOLE DOUBLET FOCUSING CHANNELS}

In this section, we explore normal mode envelope perturbations $(\delta \mathbf{P}=0)$ in alternating gradient quadrupole focusing channels. Driving perturbations with $\delta \mathbf{P} \neq$ 0 are not analyzed. Results are presented in an analogous manner to Sec. IV for solenoidal focusing. However, in contrast to Sec. IV, all results are laboratory frame and can be interpreted directly without transformation. The beam considered in this section has zero total canonical angular momentum $P_{\theta}=\left\langle x y^{\prime}-y x^{\prime}\right\rangle_{\perp}=0$ and the focusing channel has no skew couplings so that the envelope equation (4) applies (see Appendix A). Piecewise constant focusing strength $\kappa_{x}(s)=-\kappa_{y}(s) \equiv \kappa_{q}(s)$ is taken and results can be applied to realistic focusing channels with continuously varying $\kappa_{q}(s)$ using the equivalency procedures developed in Appendix C.

\section{A. Matched envelope solution}

We consider the case of a periodic, matched solution $\left(r_{j}=r_{j m}\right.$ for $\left.j=x, y\right)$ to the envelope equation (4) with equal emittances $\varepsilon_{x}=\varepsilon_{y}=\varepsilon$. A hard-edge focusing lattice is assumed with piecewise constant $\kappa_{x}(s)=$ $-\kappa_{y}(s) \equiv \kappa_{q}(s)$ as illustrated in Fig. 2(c) with lattice period $L_{p}$, quadrupole strength $\hat{\kappa}_{q}=$ const, occupancy $\eta$, and syncopation factor $\alpha$. The matched envelope 
a) $\sigma_{0}=80^{\circ}, \eta=0.6949$, and $\alpha=1 / 2$

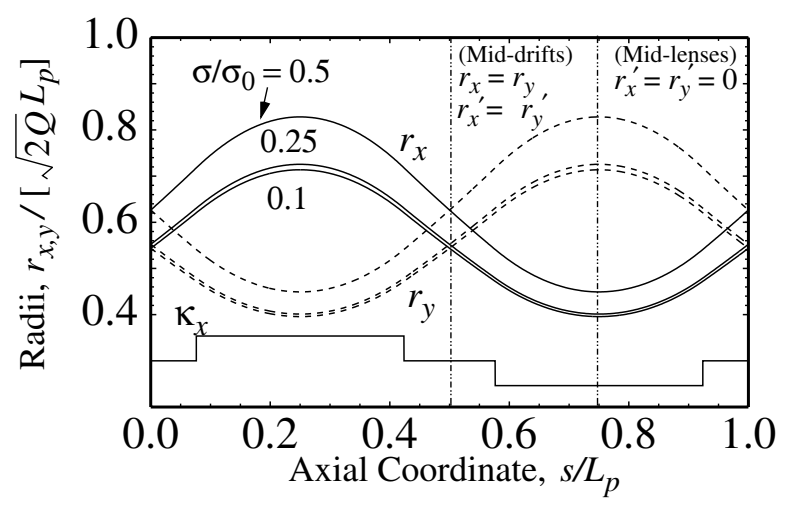

b) $\sigma_{0}=80^{\circ}, \eta=0.6949$, and $\alpha=0.1$

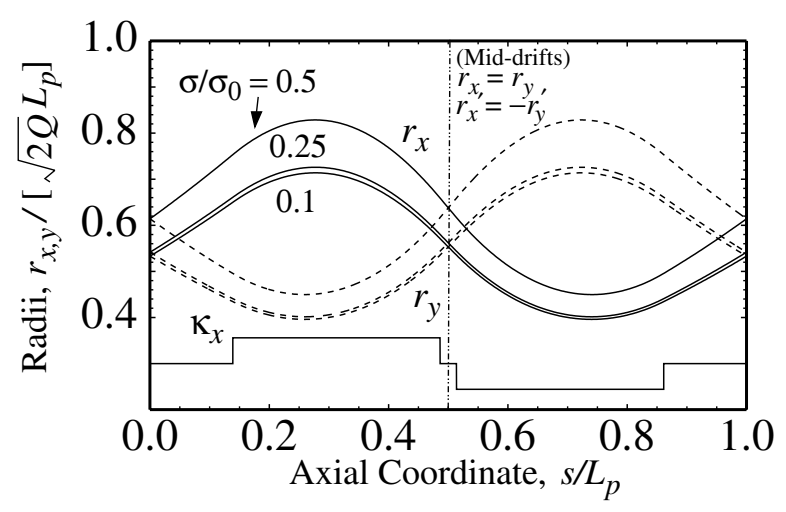

FIG. 15. Scaled matched beam envelope $r_{j} /\left(\sqrt{2 Q} L_{p}\right)$ versus normalized axial coordinate $s / L_{p}$ for one period of a hardedge quadrupole focusing channel in Fig. 2(c). Solutions are shown for $\sigma_{0}=80^{\circ}, \eta=0.6949$, and values of $\sigma / \sigma_{0}$ indicated for syncopation factors $\alpha=1 / 2$ (FODO) and $\alpha=0.1$ in (a) and (b), respectively.

solutions are parametrized by $L_{p}, \sigma_{0}, \sigma / \sigma_{0}, \eta$, and $\alpha$. Numerical solutions are plotted for one lattice period in Fig. 15 in terms of scaled envelope radii $\left[r_{j} /\left(\sqrt{2 Q} L_{p}\right)\right]$ versus normalized axial coordinate $\left(s / L_{p}\right)$ for $\alpha=1 / 2$ [FODO channel, (a)] and $\alpha=0.1$ [strong syncopation, (b)]. In these plots, the undepressed single-particle phase advance $\sigma_{0}$ is fixed, and the solution is shown for values of $\sigma / \sigma_{0}$. Approximate, analytical solutions can also be constructed using the perturbative formulation of Lee [25]. Symmetry points are indicated on the plot. Contrasting (a) and (b), observe that syncopation with $\alpha \neq 1 / 2$ reduces the symmetry of the matched envelope but that maximum radial excursions of the envelope change little. In contrast to solenoidal transport channels, at fixed $\sigma_{0}$, little variation in matched envelope structure is observed with changes in quadrupole occupancy $\eta$. The value of occupancy employed $\eta=0.6949$ corresponds to the hard-edge equivalent value of a FODO electric quadrupole lattice in the High Current Transport Experiment (HCX) at the Lawrence Berkeley National Laboratory $[30,31]$. This equivalence prescription is derived as an example application in the general discussion of fringe field equivalent models presented in Appendix C. Negligible difference in the structure of the matched beam envelope is observed between the actual focusing function $\kappa_{q}(s)$ derived from the linear applied field components of the physical lattice and the hard-edge equivalent model with piecewise constant $\kappa_{q}(s)$.

\section{B. Normal modes}

Normal mode solutions are numerically analyzed using the formulation in Sec. II F. For the matched beam solutions described in Sec. VA, mode perturbations are not simply decoupled by sum $(+)$ and difference $(-)$ coordinates $\delta r_{ \pm}=\left(r_{x} \pm r_{y}\right) / 2$ as in the case of continuous and solenoidal focusing channels analyzed in Secs. III and IV, and we employ the full coupled eigenvalue formulation based on Eq. (38) with $\kappa_{x}=-\kappa_{y}=\kappa_{q}, \varepsilon_{x}=$ $\varepsilon_{y}=\varepsilon$, and zero driving perturbations $(\delta \mathbf{P}=0)$. Eigenvalues are calculated for specified values of $\eta$ and $\alpha$ and ranges of $\sigma_{0}$ and $\sigma / \sigma_{0}$ to map out mode properties. Results we obtained are displayed in Figs. 16-20. Although sum and difference perturbations are not decoupled in quadrupole transport channels, we find that the pure modes have properties analogous to the sum (i.e., "breathing") and difference (i.e., "quadrupole") modes studied in continuous and solenoidal channels. We label envelope modes in the quadrupole focusing channel that in stable regions degenerate into quadrupole- and breathinglike perturbations with $B$ and $Q$, respectively (e.g., $\sigma_{\ell}$ with $\ell=B, Q$ for the mode phase advances), to avoid confusion with the purely decoupled sum and difference modes analyzed in Secs. III and IV.

The breathing and quadrupole mode phase advances $\sigma_{B}$ and $\sigma_{Q}$ and growth factors $\gamma_{B}$ and $\gamma_{Q}$ are contoured in Fig. 16 as a function of $\sigma_{0}$ and $\sigma / \sigma_{0}$ for $\sigma_{0} \in\left(0,180^{\circ}\right)$ and $\sigma / \sigma_{0} \in(0,1]$. Results are shown for quadrupole occupancy $\eta=0.6949$ and syncopation factors $\alpha=1 / 2$ (FODO lattice) and $\alpha=0.1$ in 16(a) and 16(b) to contrast a symmetrical FODO lattice with the case of strong syncopation. Growth factors of both the breathing $\left(\gamma_{B}>1\right)$ and quadrupole $\left(\gamma_{Q}>1\right)$ modes are superimposed on the same plots because the bands do not overlap, and growth factors of the corresponding damped modes are not plotted because damping factors are given by $1 / \gamma_{\ell}$ of the growing mode (see Fig. 4 and Table I). No instability bands are found for $\sigma_{0}<90^{\circ}$ so the growth contours are restricted to $\sigma_{0} \in\left(90^{\circ}, 180^{\circ}\right)$. In terms of the symmetry classes presented in Fig. 4, eigenvalues fall into class (a) when there is stability and class (b) (confluent resonance) or (c) (lattice resonance) when there is instability. The confluent resonance involves locking of the breathing and quadrupole modes and occurs for both FODO and syncopated $(\alpha \neq 1 / 2)$ lattices, while the lattice resonance is observed only for syncopated lattices (see the thin band to the left of the confluent resonance 


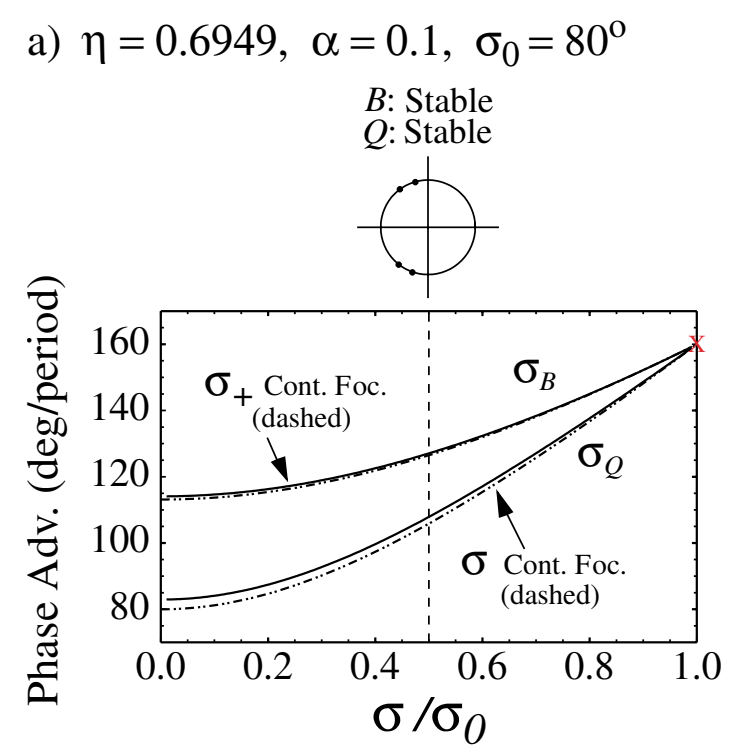

b) $\eta=0.6949, \alpha=0.1, \sigma_{0}=115^{\circ}$
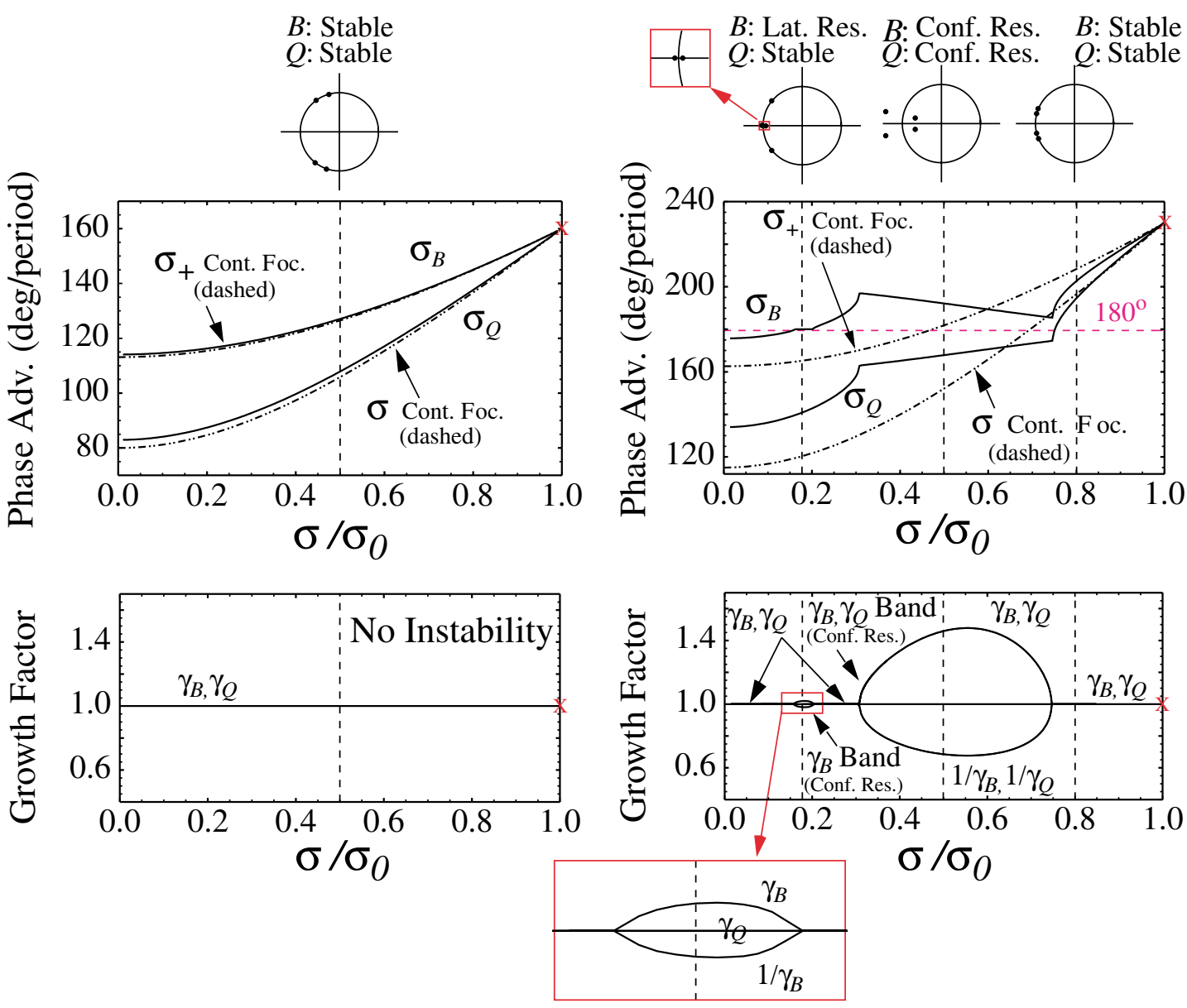

FIG. 17. (Color) Phase advance $\left(\sigma_{B}\right.$ and $\left.\sigma_{Q}\right)$ and growth factors $\left(\gamma_{B}\right.$ and $\left.\gamma_{Q}\right)$ of the breathing and quadrupole envelope modes versus $\sigma / \sigma_{0}$ for a quadrupole focusing channel with occupancy $\eta=0.6949$, syncopation factor $\alpha=0.1$, and $\sigma_{0}=80^{\circ}$ and $\sigma_{0}=$ $115^{\circ}$ in (a) and (b). Continuous focusing model predictions for $\sigma_{ \pm}$are superimposed on the phase advance plots (dashed curves). Red $\times_{s}$ mark analytical results in the zero space-charge limit. Vertical dashed lines correspond to locations of plots of complex eigenvalues $\lambda_{n}$ placed above. Red boxes denote expanded views.

To better illustrate the mode structures presented in Fig. 16, in Fig. 17 we plot the mode phase advances and growth factors for fixed $\sigma_{0}, \alpha$, and $\eta$ as a function of $\sigma / \sigma_{0}$. Curves shown correspond to the vertical dashed lines on the contour plots in Fig. 16. Complex plots of the eigenvalues $\lambda_{n}$ relative to the unit circle are superimposed at several locations to further illustrate the eigenvalue structure of the modes. Individual mode branches for the breathing and quadrupole modes are identified outside of the regions of instability using the formulation described in Sec. IIF and branches are continuously connected across bands of parametric instability. The phase advance of the breathing mode $\sigma_{B}$ is larger than the quadrupole mode $\sigma_{Q}$ for finite space charge $\left(\sigma / \sigma_{0}<1\right)$. Continuous focusing model predictions shown for the sum (i.e., breathing) and difference (i.e., quadrupole) mode phase advances $\sigma_{+}$and $\sigma_{-}$are derived from Eq. (64) using the single-particle phase advances $\sigma_{0}$ and $\sigma$ calculated from the periodically focused, matched beam. Far from bands of instability, $\sigma_{+}$and $\sigma_{-}$provide accurate estimates for the numerically calculated breathing and quadrupole mode phase advances $\sigma_{B}$ and $\sigma_{Q}$ [7]. This correspondence helps justify the identification of the mode branches being breathing- and quadrupole-like. The continuous focusing and alternating gradient model phase advances are in better agreement for lower values of $\sigma_{0}$ and weaker syncopation $(\alpha \rightarrow 1 / 2)$. Analysis in Sec. IIF [see Eq. (56)] shows that the zero space-charge limit $Q \rightarrow 0$ corresponds to stable breathing and quadrupole mode oscillations with

$$
\lim _{\sigma \rightarrow \sigma_{0}} \sigma_{\ell}=2 \sigma_{0}, \quad \lim _{\sigma \rightarrow \sigma_{0}} \gamma_{\ell}=1
$$

These limiting values are indicated on the figures and provide a partial check of results.

For FODO lattices with the same values of the particle phase advances $\sigma_{0}$ and $\sigma$, little variation is observed in 


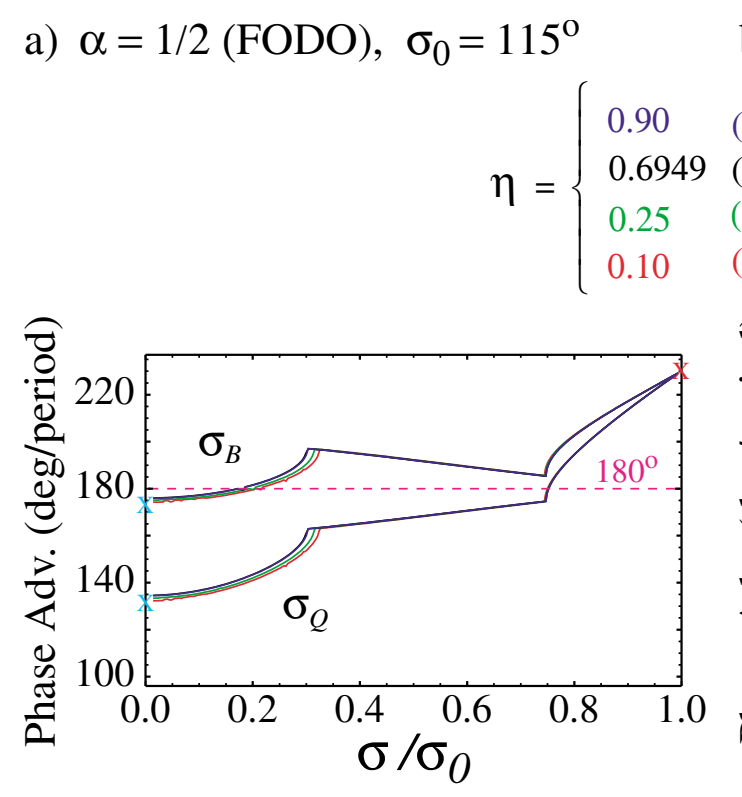

b) $\alpha=0.1, \sigma_{0}=115^{\circ}$

$$
\eta= \begin{cases}0.90 & \text { (Blue) } \\ 0.6949 & \text { (Black) } \\ 0.25 & \text { (Green) } \\ 0.10 & \text { (Red) }\end{cases}
$$
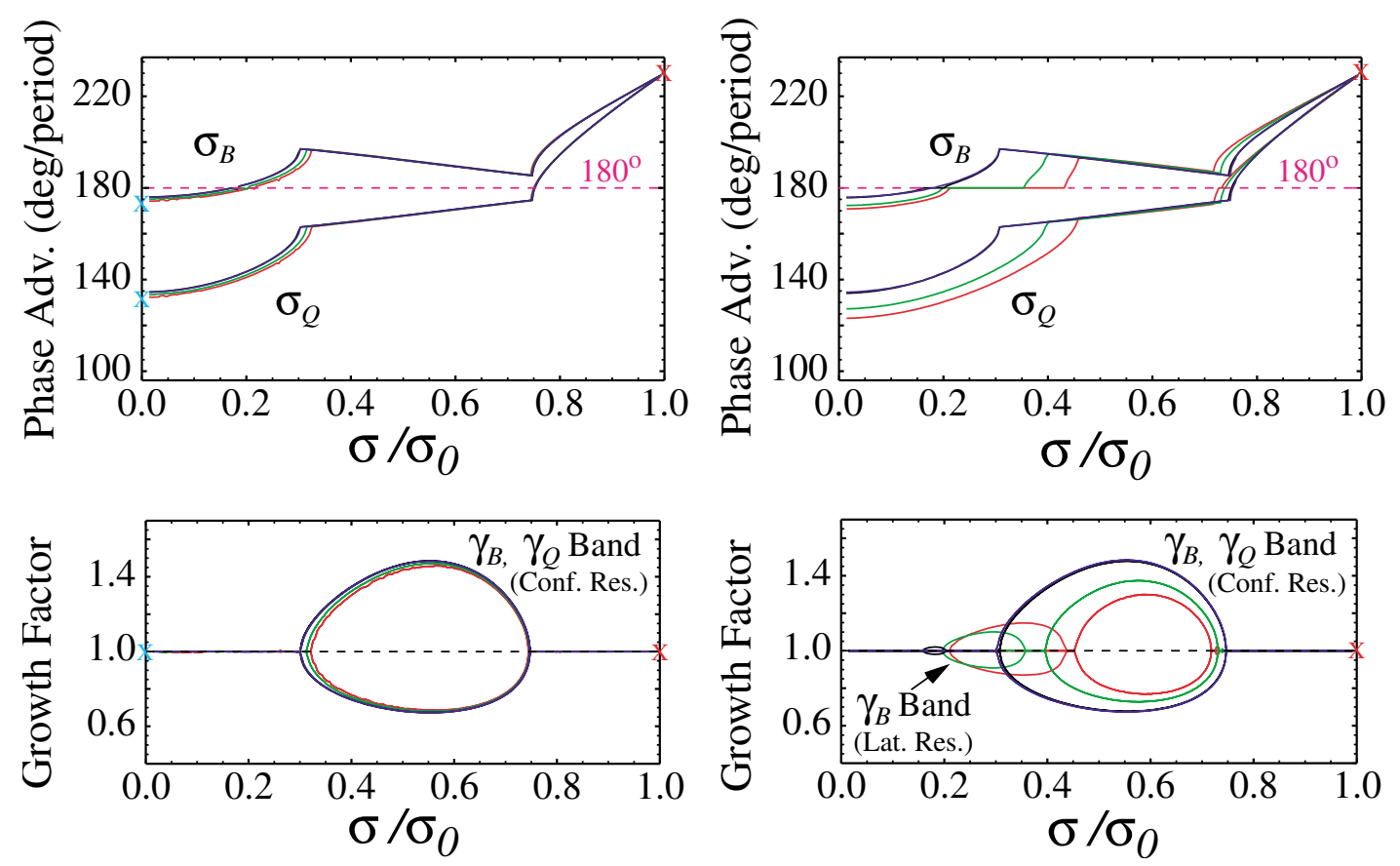

FIG. 18. (Color) Variation of mode structure with quadrupole occupancy $\eta$ and syncopation factor $\alpha$. Phase advance ( $\sigma_{B}$ and $\sigma_{Q}$ ) and growth factors $\left(\gamma_{B}\right.$ and $\left.\gamma_{Q}\right)$ of the breathing and quadrupole modes are plotted versus $\sigma / \sigma_{0}$ for $\sigma_{0}=115^{\circ}$ and the indicated values of $\eta$. Results are contrasted in (a) and (b) for $\alpha=1 / 2$ (FODO) and $\alpha=0.1$ (strong syncopation). Red $\times$ s mark analytical results in the zero space-charge limit, and cyan $\times$ s mark thin-lens limit $(\eta \rightarrow 0)$ analytical results for a FODO focusing with $\sigma / \sigma_{0}=0$.

envelope mode structure with changes in quadrupole occupancy $\eta$. On the other hand, significant variations can occur in lattices with strong syncopation ( $\alpha$ far from $1 / 2$ ). To illustrate this point, the mode phase advances $\sigma_{B}$ and $\sigma_{Q}$ and growth factors $\gamma_{B}$ and $\gamma_{Q}$ are plotted in Fig. 18 for several values of $\eta$. Results are contrasted in columns (a) and (b) for a FODO lattice $(\alpha=1 / 2)$ and a strongly syncopated lattice with $\alpha=0.1$. The small variations presented in Fig. 18(a) illustrate the insensitivity of the mode structure to changes in $\eta$ for a FODO lattice. The location and strength of the confluent resonance instability band changes only weakly with $\eta$. Even smaller variations are observed in FODO lattices when making comparisons for $\sigma_{0}$ farther from the first instability band or when comparing results for the hard-edge lattice taken here with full fringe field models calculated from physical lattices (see Appendix C). Results from eigenvalues analytically calculated in Appendix $\mathrm{H}$ for the thin-lens limit ( $\eta \rightarrow 0$ at fixed $\sigma_{0}$ ) of a FODO lattice with a fully space-charge depressed beam $(\sigma=0)$ are indicated in Fig. 18(a). The good agreement with the thinlens results is traceable to the mode structure in a FODO lattice being relatively insensitive to the quadrupole occupancy $\eta$. For FODO lattices, $\sigma_{0}$ and $\sigma$ are the most important parameters, whereas shape parameters characterizing the occupancy and fringe components of the focusing field are of much lesser importance. In contrast, for strong syncopation, it is evident from Fig. 18(b) that the mode structure can vary significantly with occupancy $\eta$. This strong variation is correlated to the existence of the second band of parametric instability (lattice resonance) for the breathing mode, which was shown in Sec. IV to vary strongly with $\eta$ in a solenoidal focusing lattice. Observe from the $\alpha=0.1$ plots in Fig. 18(b) that the instability band of the lattice resonance is weakly expressed compared to the confluent resonance band for higher lattice occupancies $\eta$, whereas it is strongly expressed for $\eta$ small. High resolution numerical studies suggest that the lattice resonance instability band disappears, becoming both thinner and weaker, as $\alpha \rightarrow 1 / 2$. 
Breathing Mode, Mid-quadrupole
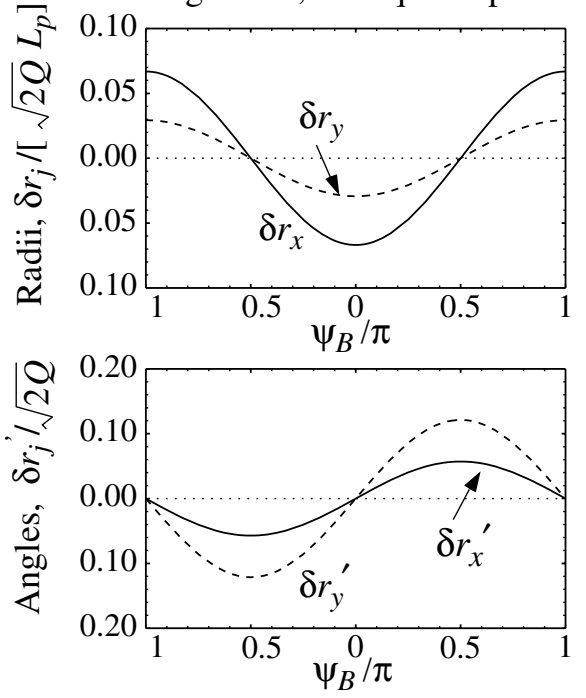

Breathing Mode, Mid-drift
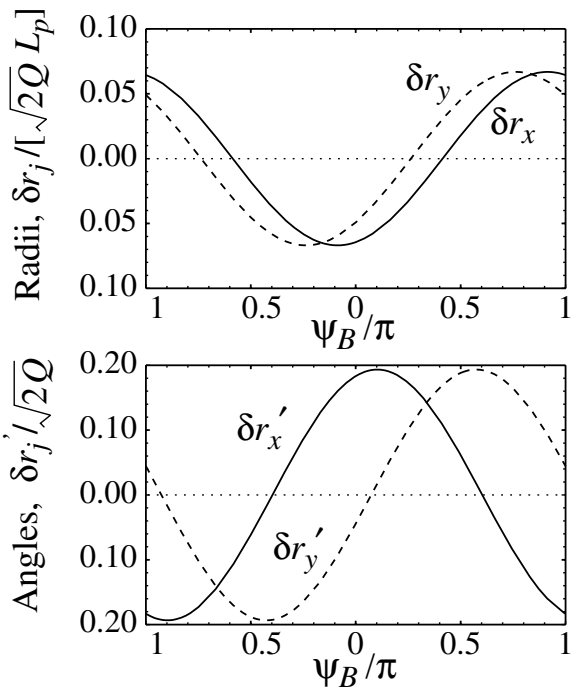

Quadrupole Mode, Mid-quadrupole
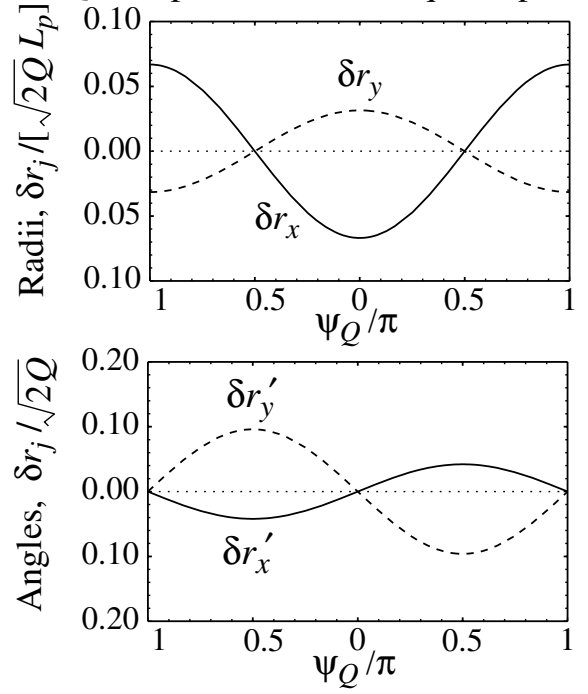

Quadrupole Mode, Mid-drift
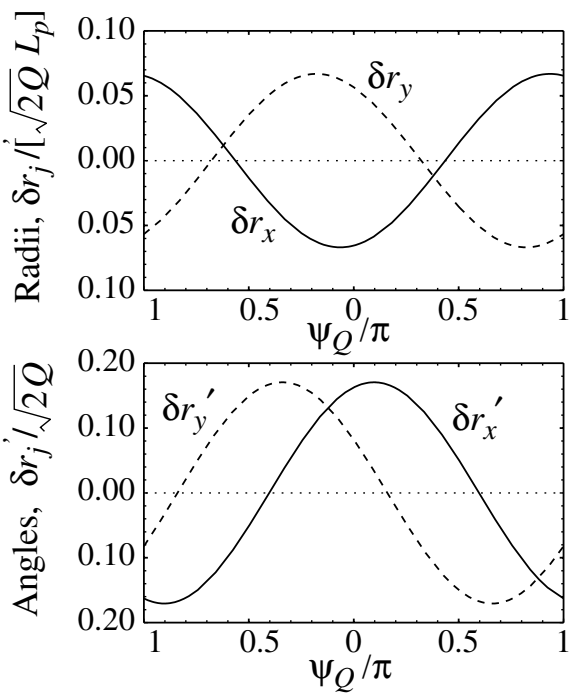

FIG. 19. Scaled pure mode launching conditions in $\delta r_{j} /\left(\sqrt{2 Q} L_{p}\right)$ and $\delta r_{j}^{\prime} / \sqrt{2 Q}(j=x, y)$ plotted versus mode phase $\psi_{\ell}(\ell=B$, $Q$ ) for stable breathing and quadrupole modes at the axial mid-drift (upper-half) and mid-quadrupole (lower-half) locations in a FODO lattice. Parameters chosen are $\eta=0.6949, \alpha=1 / 2(\mathrm{FODO}), \sigma_{0}=80^{\circ}$, and $\sigma / \sigma_{0}=0.2$.

The confluent resonance instability band has been identified as a half-integer parametric resonance between both envelope mode oscillation frequencies and the lattice, and the lattice resonance instability band has been identified as a half-integer parametric resonance between one envelope mode and the lattice [7]. This suggests that the location of the envelope bands can be crudely estimated using resonance conditions based on the breathing and quadrupole mode frequencies $\sigma_{ \pm}$derived from the continuous model with $\sigma_{+}=\sqrt{2 \sigma_{0}^{2}+2 \sigma^{2}}$ and $\sigma_{-}=$ $\sqrt{\sigma_{0}^{2}+3 \sigma^{2}}$ [see Eq. (64)] as $\left(\sigma_{+}+\sigma_{-}\right) / 2=\pi$ for the confluent resonance band and $\sigma_{+}=\pi$ for the lattice resonance band, or equivalently with $\sigma_{0}$ and $\sigma$ measured in degrees,

$$
\sqrt{2 \sigma_{0}^{2}+2 \sigma^{2}}+\sqrt{\sigma_{0}^{2}+3 \sigma^{2}}=360^{\circ}
$$

for the confluent resonance band (both breathing and quadrupole modes), and

$$
\sqrt{2 \sigma_{0}^{2}+2 \sigma^{2}}=180^{\circ}
$$

for the lattice resonance band (breathing mode). Comparing to Fig. 16, Eq. (86) roughly predicts the location of the maximum growth factor in the confluent resonance band for the breathing and quadrupole modes, and Eq. (87) roughly predicts the location of the thin lattice resonance band associated with the breathing mode in syncopated lattices. However, the formulas can- 


$$
\eta= \begin{cases}0.90 & \text { (Blue) } \\ 0.6949 & \text { (Black) } \\ 0.25 & \text { (Green) } \\ 0.10 & \text { (Red) }\end{cases}
$$

Breathing Mode, $\sigma / \sigma_{0}=0.2$

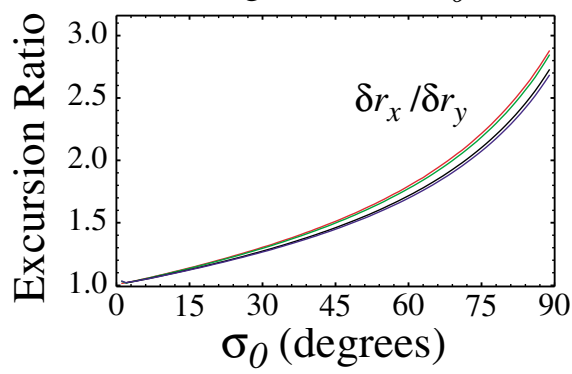

Quadrupole Mode, $\sigma / \sigma_{0}=0.2$

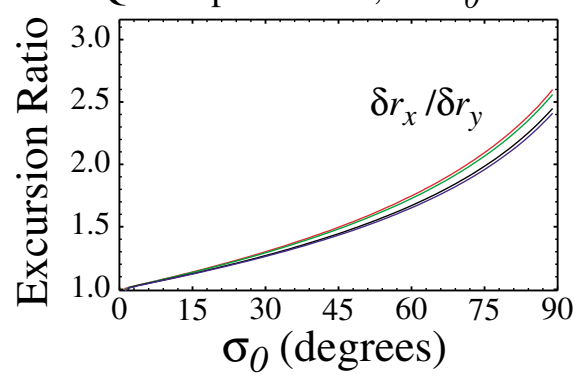

Breathing Mode, $\sigma / \sigma_{0}=0.5$

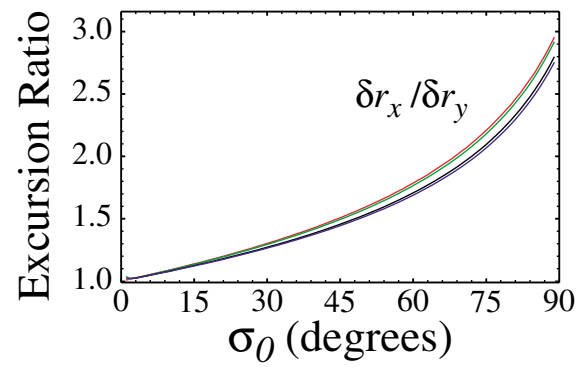

Quadrupole Mode, $\sigma / \sigma_{0}=0.5$

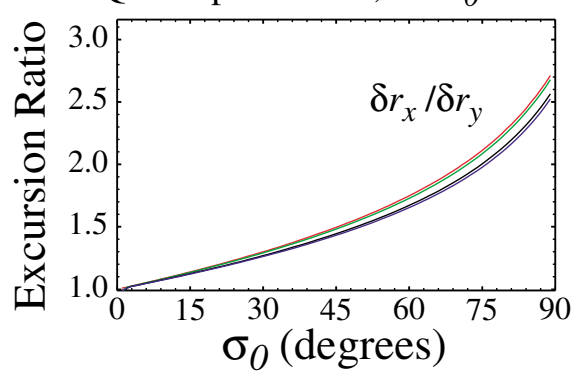

FIG. 20. (Color) Ratio of $\delta r_{x} / \delta r_{y}$ as a function of $\sigma_{0}$ for breathing (upper row) and quadrupole (lower row) mode launches at the axial midpoint of a focusing-in- $x$ quadrupole in a FODO $(\alpha=1 / 2)$ lattice. Both launch conditions have $\delta r_{x}^{\prime}=0=\delta r_{y}^{\prime}$ and are shown for $\sigma / \sigma_{0}=0.2$ (left column) and $\sigma / \sigma_{0}=0.5$ (right column) and the indicated values of $\eta$.

not capture a broad parametric nature of the instability or changes in band structure with solenoid occupancy $\eta$ or syncopation factor $\alpha$. Moreover, Eq. (87) fails to suggest the nonexistence of the band for FODO lattices with $\alpha=$ $1 / 2$. In order to provide some guidance for band locations in practical applications, parametric data were employed to calculate curve fits for the instability thresholds for a FODO lattice. A least-squares fit was carried out using a large amount of threshold data for the confluent resonance band. Elliptic and linear boundary constraint equations in $\sigma_{0}$ and $\sigma$ measured in degrees were applied with forms:

$$
\sigma^{2}+f(\eta) \sigma_{0}^{2}=\left(90^{\circ}\right)^{2}[1+f(\eta)]
$$

for the left edge of the FODO confluent resonance band, and

$$
\sigma+g(\eta) \sigma_{0}=90^{\circ}[1+g(\eta)],
$$

for the right edge of the FODO confluent resonance band. Here, $f(\eta)$ and $g(\eta)$ are functions to be determined by fitting the data, and the forms taken correspond to the most general centered ellipse and line containing the limit point $\sigma=\sigma_{0}=90^{\circ}$. We find very little variation in $\eta$ when fitting to these forms (almost to the accuracy of the numerical data) and errors are minimized by taking

$$
f(\eta)=\frac{4}{3}, \quad g(\eta)=\frac{1}{9} .
$$

Maximum errors (all $\eta$ values) in threshold prediction from Eqs. (88) $-(90)$ are $\sim 5^{\circ}$ and $\sim 2^{\circ}$ for the left and right edges of the band, respectively. No two-dimensional curve fits were attempted to understand and characterize the more intricate band changes observed in a syncopated quadrupole lattice with $\alpha \neq 1 / 2$. Generalizations of methods employed by Lee and Briggs [23] using Fourier expansions of the lattice focusing function $\kappa_{q}(s)$ might be employed to derive analytical formulas to better understand the instability thresholds. This topic is left for future research.

To further illustrate the mode structure, pure mode launching conditions in $\delta \mathbf{R}=\left(\delta r_{x}, \delta r_{x}^{\prime}, \delta r_{y}, \delta r_{y}^{\prime}\right)$ are plotted in Fig. 19 for both the breathing and the quadrupole modes. Launching conditions are plotted at axial locations corresponding to matched beam symmetry points in a FODO lattice: the axial mid-drift with focusing-in- $x$ and defocusing-in- $x$ quadrupoles at smaller and larger $s$ values, and mid-quadrupole within a focusing-in- $x$ quadrupole. Launching conditions at the other axial mid-drift and mid-defocusing-quadrupole 
locations are simply derivable by symmetry from the plots shown. The launching conditions employed are described in Table I and coordinates and angles are scaled as $\delta r_{j} /\left(\sqrt{2} \bar{Q} L_{p}\right)$ and $\delta r_{j}^{\prime} / \sqrt{2} \bar{Q}$. Mode amplitudes $A_{\ell}$ with $\ell=B, Q$ are selected so that the maximum excursions in $\left|\delta r_{j}\right|$ for the pure mode are $10 \%$ of the average matched beam radius (i.e., $\max \left[\delta r_{j}\left(\psi_{\ell}\right)\right]=$ $\left.0.1\left(1 / L_{p}\right) \int_{0}^{L_{p}} d s \sqrt{r_{x m}} r_{y m}\right)$ and launching conditions are plotted versus mode phase $\psi_{\ell}$ for $-\pi \leq \psi_{\ell} \leq \pi$ (the absolute values of the phases are arbitrary). Parameters chosen for Fig. 19 correspond to stable modes with eigenvalues and launching conditions falling into class (a) in Fig. 4 and Table I. To launch a stable mode with a prescribed maximal excursion in $\delta r_{j}(s)$, it suffices to select a particular axial location $s=s_{i}$ of interest, calculate needed eigenvectors for the full lattice period as a function of $s$, calculate the maximum value of $\left|\delta r_{j}\left(s_{i}\right)\right|$ with respect to both $s$ and the mode phase $\psi_{\ell}$, and then scale the mode amplitude $A_{\ell}$ such that this maximum value equals the prescribed excursion. Outside of exceptional cases where the mode phase advance $\sigma_{\ell}$ is a rational fraction of $2 \pi$ (i.e., the lattice phase advance), this maximal excursion will be approached arbitrarily close as the beam propagates through the periodic lattice.

Comparing the launching conditions in Fig. 19, we observe that initial conditions in $\delta r_{j}$ and $\delta r_{j}^{\prime}$ for pure quadrupole or breathing mode launches vary significantly in form with the axial location within the lattice period. This contrasts the situation for the decoupled sum and difference modes analyzed in Secs. III and IV for continuous and solenoidal focusing channels. In these decoupled cases the launching conditions are the same form independent of axial location [see Eqs. (53) and (54)] and, to launch a given mode amplitude, only the relative projections on the normal mode coordinates and angles vary with axial location in the lattice period. The decoupled mode launch conditions in Eqs. (53) and (54) are a poor approximation to the pure breathing and quadrupole mode launching conditions for an alternating gradient channel even though the modes have rough breathing and quadrupole symmetry for specific launch phases and axial coordinate locations. Thus the use of the continuous model as a guide for pure mode launchings in alternating gradient focusing lattices (this has been commonly done in simulation studies, etc.) can result in significant launching errors with unwanted projections on the mode desired to be suppressed. A useful reduced prescription for stable pure mode launching $\left(\sigma_{0}<90^{\circ}\right)$ at the midaxial location of a focusing-in- $x$ quadrupole in a FODO lattice (where the $x$ excursion of $r_{x m}$ is maximum and the $y$ excursion in $r_{y m}$ is minimum) is to take $\delta r_{x}$ to be a specified fraction of $\delta r_{y}$ and zero perturbed angles $\left(\delta r_{j}^{\prime}=0\right)$. By symmetry, analogous launching conditions with the same fractions apply at the midaxial location of a focusing-in- $y$ quadrupole. The numerically calculated excursion ratio $\delta r_{x} / \delta r_{y}$ needed for this pure mode launch is illustrated in Fig. 20. The excursion ratio is plotted separately for pure breathing and quadrupole mode launches as a function of $\sigma_{0}$ for several values of quadrupole occupancy $\eta$ and $\sigma / \sigma_{0}$. Observe that the excursion ratio varies weakly in both occupancy $\eta$ and space-charge depression $\sigma / \sigma_{0}$ relative to variations in $\sigma_{0}$. For approximate launches these variations in $\eta$ and $\sigma / \sigma_{0}$ can sometimes be neglected and the curves shown can then be applied for values of these parameters not shown. Observe that for decreasing values of $\sigma_{0}$ the excursion ratio approaches unity. This is expected because alternating gradient focusing is better approximated by continuous focusing for lower values of $\sigma_{0}$ [3]. The launching conditions illustrated in Fig. 20 hold only for a lattice with FODO symmetry $(\alpha=1 / 2)$ and are violated if the lattice is syncopated with $\alpha \neq 1 / 2$.

\section{CONCLUSIONS}

This study extends the present understanding of the stability properties of mismatch modes supported by the KV envelope equations describing the transverse evolution of a coasting, unbunched beam envelope. Generalized linear perturbation equations were derived that describe mismatch evolution resulting from both the usually analyzed mismatch source resulting from deviations in envelope coordinates from the periodic matched beam condition as well as driving errors in focusing, perveance, and emittance. Important general properties of undriven envelope modes were analyzed including symmetry classes, zero space-charge limits, and phasespace invariants. The linear perturbation equations were solved in general for continuous focusing channels, and example solutions presented illustrate important differences in the action of adiabatic and sudden driving errors as well as the scaling of contributions to the breathing and quadrupole mismatch modes from the driving errors. The normal modes in the absence of driving errors were also parametrically analyzed in hard-edge, periodic solenoidal and alternating gradient quadrupole doublet focusing channels. This analysis extended earlier work by Struckmeier and Reiser [7] by systematically identifying possible envelope instabilities over a wide range of system parameters, thereby providing valuable data for practical applications. For solenoidal focusing, a systematic derivation of the consistent $\mathrm{KV}$ distribution identified invariant emittances and the appropriate Larmor-frame transformation to interpret the analysis under, both of which are necessary for elliptical beam perturbations and were overlooked in earlier studies. It was shown that the solenoid occupancy strongly influences stability properties and that quadrupole symmetry envelope perturbations are generally less critical than breathing symmetry envelope perturbations for intense beam transport, thereby simplifying design considerations. For quadrupole doublet focusing, a previously overlooked mode was 
found associated with syncopated lattices where one drift between quadrupoles is shorter than the other. For both solenoidal and quadrupole focusing channels, pure mismatch mode launching conditions were constructed. Exact launching conditions were specified for pure breathing and quadrupole envelope mode evolutions in solenoidal channels and approximate launch conditions were formulated for quadrupole focusing channels at lattice symmetry points. These new results provide insight on beam envelope control and have important applications in envelope matching including possible halo mitigation through breathing mode suppression and in simulation studies.

The present study is limited in that only specific (though representative) cases of linear solenoidal and quadrupole focusing lattices have been analyzed. Also, analysis of properties of mode evolutions due to driving term errors in focusing, perveance, and emittance in periodic focusing channels is left for future study.

\section{ACKNOWLEDGMENTS}

The authors wish to thank J. J. Barnard and E. P. Lee for useful discussions, P. A. Seidl and M. Leitner for help with the example application of the fringe field equivalent model to the High Current Experiment, and D. P. Grote and J.-L. Vay for help with the WARP code in carrying out applied field simulations of the High Current Experiment lattice. This research was performed under the auspices of the U.S. Department of Energy by the University of California at the Lawrence Livermore and Lawrence Berkeley National Laboratories under Contracts No. W-7405-Eng-48 and No. DE-AC03-76SF00098.

\section{APPENDIX A: LARMOR-FRAME TRANSFORMATIONS FOR ANALYSIS OF SOLENOIDAL FOCUSING SYSTEMS AND SELF- CONSISTENT KV DISTRIBUTION FUNCTIONS}

In situations where there is an $s$-varying solenoidal magnetic field $\mathbf{B}(s)=-\left[B_{z}^{\prime}(s) / 2\right](x \hat{\mathbf{x}}+y \hat{\mathbf{y}})+B_{z}(s) \hat{\mathbf{z}}$ in addition to (or in place of) other physical laboratoryframe focusing forces described by $\kappa_{x}(s)$ and $\kappa_{y}(s)$ (e.g., electric or magnetic quadrupoles or a uniform, partially neutralizing background), the transverse equations of motion of a beam particle can be expressed as $[3,32]$

$$
\begin{aligned}
& x^{\prime \prime}+\kappa_{x} x-k_{L}^{\prime} y-2 k_{L} y^{\prime}+\frac{q}{m \gamma_{b}^{3} \beta_{b}^{2} c^{2}} \frac{\partial \phi}{\partial x}=0, \\
& y^{\prime \prime}+\kappa_{y} y+k_{L}^{\prime} x+2 k_{L} x^{\prime}+\frac{q}{m \gamma_{b}^{3} \beta_{b}^{2} c^{2}} \frac{\partial \phi}{\partial y}=0 .
\end{aligned}
$$

Here,

$$
k_{L}(s)=\frac{q B_{z}(s)}{2 m \gamma_{b} \beta_{b} c}=\frac{\omega_{c}}{2 \gamma_{b} \beta_{b} c}
$$

is the Larmor wave number, $\omega_{c}=q B_{z} / m$ is the cyclotron frequency, and $-\partial \phi / \partial x$ and $-\partial \phi / \partial y$ are the $x$ and $y$ components of the self-electric field of the beam in the electrostatic approximation. For solenoidal focusing $\kappa_{x}=\kappa_{y}=0$ and $\int_{0}^{L_{p}} d s k_{L}^{2}(s) \neq 0$. Note that Eq. (A1) reduces to Eq. (1) for $k_{L}=0$ and $\left(q / m \gamma_{b}^{3} \beta_{b}^{2} c^{2}\right) \partial \phi / \partial x=$ $-Q x /\left[\left(r_{x}+r_{y}\right) r_{x}\right] \quad$ and $\quad\left(q / m \gamma_{b}^{3} \beta_{b}^{2} c^{2}\right) \partial \phi / \partial y=$ $-Q y /\left[\left(r_{x}+r_{y}\right) r_{y}\right]$, corresponding to the interior fields of a uniform density elliptical beam in free space with principal axis radii $r_{x}$ and $r_{y}$ oriented along the $x$ and $y$ coordinate axes.

For solenoidal focusing, the cross-coupled form of Eq. (A1) results in a macroscopic rotation of the beam about the longitudinal axis. Because of this, we analyze the particle equations in a $\tilde{x}-\tilde{y}$ coordinate system rotated by an angle $\tilde{\psi}(s)$ relative to the $x-y$ laboratory frame as indicated in Fig. 21 with

$$
\begin{aligned}
& \tilde{x}=x \cos \tilde{\psi}+y \sin \tilde{\psi}, \\
& \tilde{y}=-x \sin \tilde{\psi}+y \cos \tilde{\psi} .
\end{aligned}
$$

Henceforth, we use tildes to denote quantities measured in the rotating frame. For a uniform density elliptical beam with (possibly evolving) principal axes $\tilde{r}_{\tilde{x}}=$ $2\left\langle\tilde{x}^{2}\right\rangle_{\perp}$ and $\tilde{r}_{\tilde{y}}=2\left\langle\tilde{y}^{2}\right\rangle_{\perp}$ along the rotating $\tilde{x}$ and $\tilde{y}$ coordinate axes (see Fig. 21), the self-field forces within the beam are given by

$$
\begin{aligned}
-\frac{q}{m \gamma_{b}^{3} \beta_{b}^{2} c^{2}} \frac{\partial \phi}{\partial x} & =\frac{2 Q}{\tilde{r}_{\tilde{x}}+\tilde{r}_{\tilde{y}}}\left(\frac{\tilde{x}}{\tilde{r}_{\tilde{x}}} \cos \tilde{\psi}-\frac{\tilde{y}}{\tilde{r}_{\tilde{y}}} \sin \tilde{\psi}\right), \\
-\frac{q}{m \gamma_{b}^{3} \beta_{b}^{2} c^{2}} \frac{\partial \phi}{\partial y} & =\frac{2 Q}{\tilde{r}_{\tilde{x}}+\tilde{r}_{\tilde{y}}}\left(\frac{\tilde{x}}{\tilde{r}_{\tilde{x}}} \sin \tilde{\psi}+\frac{\tilde{y}}{\tilde{r}_{\tilde{y}}} \cos \tilde{\psi}\right) .
\end{aligned}
$$

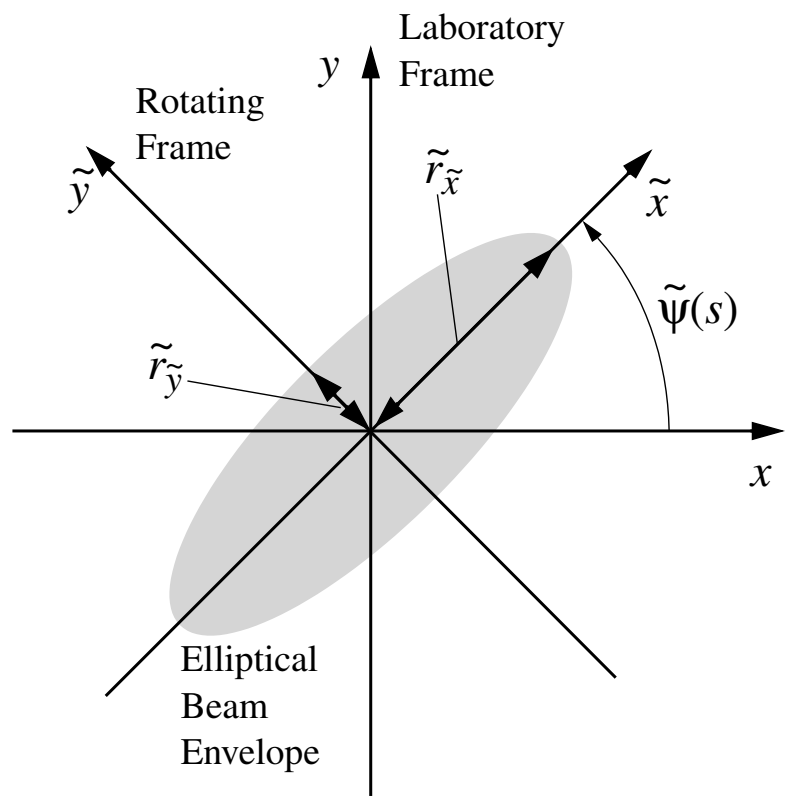

FIG. 21. Solenoidally focused elliptical envelope beam with principal axis radii $\tilde{r}_{\tilde{x}}$ and $\tilde{r}_{\tilde{y}}$ aligned along $\tilde{x}$ and $\tilde{y}$ coordinate axes that are rotated by an angle $\tilde{\psi}(s)$ relative to the laboratory frame. 
Paralleling the analysis of Wiedemann [32], we find that $\tilde{\psi}$ can be selected such that the principal axes of the uniform density elliptical beam remain aligned with the $\tilde{x}$ and $\tilde{y}$ axes as the elliptical beam evolves. This occurs when $\tilde{\psi}$ satisfies the "Larmor-frame" condition $\tilde{\psi}^{\prime}=$ $-k_{L}$ with the initial condition $\tilde{\psi}\left(s=s_{i}\right)=0$, or equivalently that

$$
\tilde{\psi}=-\int_{s_{i}}^{s} d \bar{s} k_{L}(\bar{s})
$$

For this choice of $\tilde{\psi}$, the particle equations of motion (A1) can be simply expressed in the rotating Larmor frame as

$$
\begin{aligned}
& \tilde{x}^{\prime \prime}+k_{L}^{2} \tilde{x}-\frac{2 Q}{\left(\tilde{r}_{\tilde{x}}+\tilde{r}_{\tilde{y}}\right) \tilde{r}_{\tilde{x}}} \tilde{x}=0, \\
& \tilde{y}^{\prime \prime}+k_{L}^{2} \tilde{y}-\frac{2 Q}{\left(\tilde{r}_{\tilde{x}}+\tilde{r}_{\tilde{y}}\right) \tilde{r}_{\tilde{y}}} \tilde{y}=0 .
\end{aligned}
$$

Consistent with this Larmor transform, the particle angles $x^{\prime}$ and $y^{\prime}$ transform as

$\tilde{x}^{\prime}=x^{\prime} \cos \tilde{\psi}+y^{\prime} \sin \tilde{\psi}+k_{L}(x \sin \tilde{\psi}-y \cos \tilde{\psi})$,

$\tilde{y}^{\prime}=-x^{\prime} \sin \tilde{\psi}+y^{\prime} \cos \tilde{\psi}+k_{L}(x \cos \tilde{\psi}+y \sin \tilde{\psi})$.

The particle equations of motion (A6) in the rotating Larmor-frame variables are the same form as the laboratory-frame equations of motion (1) with $\kappa_{x}=\kappa_{y} \rightarrow k_{L}^{2}$. Therefore, the usual KV analysis [33] involving transformations to phase-space invariants that are quadratic forms in conjugate phase-space variables can be employed in the rotating frame to construct a selfconsistent, KV equilibrium solution to the Vlasov equation. In the Larmor frame, the Vlasov equation for the single-particle distribution function $\tilde{f}\left(\tilde{x}, \tilde{x}^{\prime}, \tilde{y}, \tilde{y}^{\prime}\right)$ consistent with the particle equations of motion (A6) can be expressed as

$$
\begin{aligned}
\frac{d}{d s} \tilde{f}= & \frac{\partial}{\partial s} \tilde{f}+\tilde{x}^{\prime} \frac{\partial}{\partial \tilde{x}} \tilde{f}+\tilde{y}^{\prime} \frac{\partial}{\partial \tilde{y}} \tilde{f}+\left(-k_{L}^{2} \tilde{x}+\frac{2 Q \tilde{x}}{\left(\tilde{r}_{\tilde{x}}+\tilde{r}_{\tilde{y}}\right) \tilde{r}_{\tilde{x}}}\right) \frac{\partial}{\partial \tilde{x}^{\prime}} \tilde{f} \\
& +\left[-k_{L}^{2} \tilde{y}+\frac{2 Q \tilde{y}}{\left(\tilde{r}_{\tilde{x}}+\tilde{r}_{\tilde{y}}\right) \tilde{r}_{\tilde{y}}}\right] \frac{\partial}{\partial \tilde{y}^{\prime}} \tilde{f}=0 .
\end{aligned}
$$

The KV equilibrium distribution $\tilde{f}$ satisfying the Vlasov equation can be written as

$$
\begin{aligned}
& \tilde{f}\left(\tilde{x}, \tilde{x}^{\prime}, \tilde{y}, \tilde{y}^{\prime}\right)=\frac{\lambda}{q \pi^{2} \tilde{\varepsilon}_{\tilde{x}} \tilde{\varepsilon}_{\tilde{y}} \delta[}\left(\frac{\tilde{x}}{\tilde{r}_{\tilde{x}}}\right)^{2}+\frac{\left(\tilde{r}_{\tilde{x}} \tilde{x}^{\prime}-\tilde{r}_{\tilde{x}}^{\prime} \tilde{x}\right)^{2}}{\tilde{\varepsilon}_{\tilde{x}}^{2}}+\left(\frac{\tilde{y}}{\tilde{r}_{\tilde{y}}}\right)^{2} \\
&\left.+\frac{\left(\tilde{r}_{\tilde{y}} \tilde{y}^{\prime}-\tilde{r}_{\tilde{y}}^{\prime} \tilde{y}\right)^{2}}{\tilde{\varepsilon}_{\tilde{y}}^{2}}-1\right], \quad \text { (A9) }
\end{aligned}
$$

where $\delta(x)$ is the Dirac delta function, and $\tilde{\varepsilon}_{\tilde{x}}$ and $\tilde{\varepsilon}_{\tilde{y}}$ are conserved rms emittances of the uniform density beam in the rotating frame defined by

$$
\begin{aligned}
& \tilde{\varepsilon}_{\tilde{x}} \equiv 4\left[\left\langle\tilde{x}^{2}\right\rangle_{\perp}\left\langle\tilde{x}^{\prime 2}\right\rangle_{\perp}-\left\langle\tilde{x} \tilde{x}^{\prime}\right\rangle_{\perp}^{2}\right]^{1 / 2}=\mathrm{const}, \\
& \tilde{\varepsilon}_{\tilde{y}} \equiv 4\left[\left\langle\tilde{y}^{2}\right\rangle_{\perp}\left\langle\tilde{y}^{\prime 2}\right\rangle_{\perp}-\left\langle\tilde{y} \tilde{y}^{\prime}\right\rangle_{\perp}^{2}\right]^{1 / 2}=\mathrm{const},
\end{aligned}
$$

and the envelope radii $\tilde{r}_{\tilde{x}}=2 \sqrt{\langle\tilde{x}\rangle_{\perp}}$ and $\tilde{r}_{\tilde{y}}=2 \sqrt{\langle\tilde{y}\rangle_{\perp}}$ obey the envelope equations

$$
\begin{aligned}
& \tilde{r}_{\tilde{x}}^{\prime \prime}+k_{L}^{2} \tilde{r}_{\tilde{x}}-\frac{2 Q}{\tilde{r}_{\tilde{x}}+\tilde{r}_{\tilde{y}}}-\frac{\tilde{\varepsilon}_{\tilde{x}}^{2}}{\tilde{r}_{\tilde{x}}^{3}}=0, \\
& \tilde{r}_{\tilde{y}}^{\prime \prime}+k_{L}^{2} \tilde{r}_{\tilde{y}}-\frac{2 Q}{\tilde{r}_{\tilde{x}}+\tilde{r}_{\tilde{y}}}-\frac{\tilde{\varepsilon}_{\tilde{y}}^{2}}{\tilde{r}_{\tilde{y}}^{3}}=0 .
\end{aligned}
$$

If the tildes are dropped, Eq. (A9) can also be applied to describe the laboratory-frame KV distribution appropriate for quadrupole or continuous focusing channels that is consistent with $\varepsilon_{x}=$ const, $\varepsilon_{y}=$ const, and the envelope equation (4) [1,3] for the evolution of $r_{x}=2 \sqrt{\left\langle x^{2}\right\rangle_{\perp}}$ and $r_{y}=2 \sqrt{\left\langle y^{2}\right\rangle_{\perp}}$.

The laboratory-frame envelope equation (4) is identical to the Larmor-frame envelope equations (A11) under the substitutions

$$
\begin{aligned}
\kappa_{x}=\kappa_{y} & \rightarrow k_{L}^{2}=\left(\frac{\omega_{c}}{2 \gamma_{b} \beta_{b} c}\right)^{2}, \\
r_{x} & \rightarrow \tilde{r}_{\tilde{x}} \\
r_{y} & \rightarrow \tilde{r}_{\tilde{y}} \\
\varepsilon_{x} & \rightarrow \tilde{\varepsilon}_{\tilde{x}} \\
\varepsilon_{y} & \rightarrow \tilde{\varepsilon}_{\tilde{y}} .
\end{aligned}
$$

Therefore, we can apply the laboratory-frame envelope equation (4) to analyze the evolution of an elliptical beam envelope in a solenoidally focused transport channel provided we take $\kappa_{x}=\kappa_{y}=k_{L}^{2}$ and interpret the results as applying in the rotating Larmor frame defined by Eqs. (A3) and (A5). This correspondence also requires that the stationary laboratory-frame $x-y$ coordinate system be chosen to lie along the principal axes of the initial $\left(s=s_{i}\right)$ elliptical perturbations with initial values $r_{x}\left(s_{i}\right)=2 \sqrt{\left\langle x^{2}\left(s_{i}\right)\right\rangle_{\perp}}=\tilde{r}_{\tilde{x}}\left(s_{i}\right)$ and $r_{y}\left(s_{i}\right)=2 \sqrt{\left\langle y^{2}\left(s_{i}\right)\right\rangle_{\perp}}=$ $\tilde{r}_{\tilde{y}}\left(s_{i}\right)$, and the particle phase advances (i.e., $\sigma_{0}, \sigma_{x}$ and $\sigma_{y}$ ) must also be interpreted as being defined in the rotating Larmor frame.

Moment calculations verify that the KV distribution (A9) is self-consistent and help elucidate the structure of the rotating equilibrium. The Larmor-frame transformation defined by Eqs. (A3) and (A7) is canonical and phasespace area preserving, with $d x d y=d \tilde{x} d \tilde{y}$ and $d x^{\prime} d y^{\prime}=$ $d \tilde{x}^{\prime} d \tilde{y}^{\prime}$. Hence the distribution transforms to the lab frame as

$$
\tilde{f}\left(\tilde{x}, \tilde{x}^{\prime}, \tilde{y}, \tilde{y}^{\prime}\right)=f\left(x, x^{\prime}, y, y^{\prime}\right) .
$$

The rotating-frame density $\tilde{n}=\int d^{2} \tilde{x}^{\prime} \tilde{f}$ is equal to the lab frame density, i.e.,

$$
\tilde{n}(\tilde{x}, \tilde{y})=n(x, y)=\int d^{2} x^{\prime} f
$$

and can be calculated as 
TABLE II. Moments of the KV distributions for an elliptical beam with $P_{\theta}=0$ [Eq. (A9)] and an axisymmetric beam with $P_{\theta} \neq 0$ and $-1<2 P_{\theta} / \tilde{\varepsilon}<1$ [Eq. (A21)]. All second-order moments not listed vanish (i.e., $\left.\int d^{2} \tilde{x}^{\prime} \tilde{x} \tilde{y} \tilde{f}=0,\langle\tilde{x} \tilde{y}\rangle_{\perp}=0\right)$ for both distributions.

\begin{tabular}{|c|c|c|}
\hline \multirow[b]{2}{*}{ Moment } & \multicolumn{2}{|c|}{ KV Distributions } \\
\hline & Elliptical with $P_{\theta}=0$ [Eq. (A9)] & Axisymmetric with $P_{\theta} \neq 0$ [Eq. (A21)] \\
\hline $\int d^{2} \tilde{x}^{\prime} \tilde{x}^{\prime} \tilde{f}$ & $\tilde{r}_{\tilde{x}}^{\prime} \frac{\tilde{x}}{\tilde{r}_{\tilde{n}}} \tilde{n}$ & $\left(\frac{\tilde{r}_{b}^{\prime}}{\tilde{r}_{h}} \tilde{x}-2 \frac{P_{\theta}}{\tilde{r}^{2}} \tilde{y}\right) \tilde{n}$ \\
\hline $\int d^{2} \tilde{x}^{\prime} \tilde{y}^{\prime} \tilde{f}$ & $\tilde{r}_{\tilde{y}}^{\prime} \frac{\tilde{y}}{\tilde{r}_{\tilde{y}}} \tilde{n}$ & $\left(\frac{\tilde{r}_{b}^{\prime}}{\tilde{r}_{b}} \tilde{y}+2 \frac{P_{\theta}^{b}}{\tilde{r}_{b}^{2}} \tilde{x}\right) \tilde{n}$ \\
\hline $\int d^{2} \tilde{x}^{\prime} \tilde{x}^{\prime 2} \tilde{f}$ & {$\left[\frac{\tilde{\varepsilon}_{\tilde{x}}^{2}}{2 \tilde{r}_{\tilde{\tilde{n}}}^{2}}\left(1-\frac{\tilde{x}^{2}}{\tilde{r}_{\tilde{x}}^{2}}-\frac{\tilde{y}^{2}}{\tilde{r}_{\tilde{r}}^{2}}\right)+\tilde{r}_{\tilde{x}}^{\prime 2} \tilde{\tilde{x}}^{2} \tilde{r}_{\tilde{x}}^{2}\right] \tilde{n}$} & {$\left[\frac{\tilde{r}_{b}^{\prime 2}}{\tilde{r}_{b}^{2}} \tilde{\boldsymbol{x}}^{2}+\frac{4 P_{\theta}}{\tilde{r}_{b}^{3}}\left(\frac{P_{\theta} \tilde{y}}{\tilde{r}_{b}}-\tilde{r}_{b}^{\prime}\right) \tilde{y}+\frac{\tilde{\varepsilon}^{b}}{2 \tilde{r}_{t}^{2}}\left(1-\frac{4 P_{\theta}^{2}}{\tilde{\varepsilon}^{2}}\right)\left(1-\frac{\tilde{x}^{2}}{\tilde{r}_{b}^{2}}-\frac{\tilde{y}^{2}}{\tilde{r}_{b}^{2}}\right)\right] \tilde{n}$} \\
\hline $\int d^{2} \tilde{x}^{\prime} \tilde{y}^{\prime 2} \tilde{f}$ & {$\left[\frac{\tilde{\varepsilon}_{\tilde{y}}^{2}}{2 \tilde{r}_{\tilde{n}}^{2}}\left(1-\frac{\tilde{x}^{2}}{\tilde{r}_{\tilde{r}}^{2}}-\frac{\tilde{y}^{2}}{\tilde{r}^{2}}\right)+\tilde{r}_{\tilde{y}}^{\prime 2} \tilde{y}_{\tilde{r}}^{2}\right] \tilde{n}$} & {$\left[\frac{\tilde{r}_{b}^{2}}{\tilde{r}^{2}} \tilde{y}^{2}+\frac{4 P_{\theta}}{\tilde{r}^{3}}\left(\frac{P_{\theta} \tilde{x}}{\tilde{r}_{b}}+\tilde{r}_{b}^{\prime}\right) \tilde{x}+\frac{\tilde{\varepsilon}^{2}}{\tilde{r}_{\tilde{r}}^{2}}\left(1-\frac{4 P_{\theta}^{2}}{\tilde{\varepsilon}^{2}}\right)\left(1-\frac{\tilde{x}^{2}}{\tilde{r}^{2}}-\frac{\tilde{y}^{2}}{\tilde{r}^{2}}\right)\right] \tilde{n}$} \\
\hline $\int d^{2} \tilde{x}^{\prime} \tilde{x} \tilde{x}^{\prime} \tilde{f}$ & 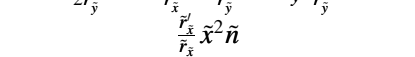 & $\left(\frac{\tilde{r}_{b}^{\prime}}{\tilde{r}_{b}} \tilde{x}-\frac{2 P_{\theta}}{\tilde{r}_{b}^{2}} \tilde{y}\right) \tilde{x} \tilde{n}$ \\
\hline $\int d^{2} \tilde{x}^{\prime} \tilde{y} \tilde{y}^{\prime} \tilde{f}$ & $\frac{\tilde{r}_{y}^{x}}{\tilde{r}_{\tilde{y}}^{x}} \tilde{y}^{2} \tilde{n}$ & $\left(\frac{\tilde{r}_{b}^{b}}{\tilde{r}_{b}} \tilde{y}+\frac{2 P_{\theta}^{b}}{\tilde{r}_{b}^{2}} \tilde{x}\right) \tilde{y} \tilde{n}$ \\
\hline $\int d^{2} \tilde{x}^{\prime}\left(\tilde{x} \tilde{y}^{\prime}-\tilde{y} \tilde{x}^{\prime}\right) \tilde{f}$ & 0 & $\frac{2 P_{\theta}}{\tilde{r}_{b}^{2}}\left(\tilde{x}^{2}+\tilde{y}^{2}\right) \tilde{n}$ \\
\hline$\left\langle\tilde{x}^{2}\right\rangle_{\perp}$ & $\frac{\tilde{r}_{\tilde{x}}^{2}}{4}$ & $\frac{\tilde{r}_{b}^{2}}{4}$ \\
\hline$\left\langle\tilde{y}^{2}\right\rangle_{\perp}$ & $\begin{aligned} \frac{\tilde{r}_{\tilde{y}}^{2}}{4} \\
\end{aligned}$ & $\frac{\tilde{r}_{b}^{2}}{4}$ \\
\hline$\left\langle\tilde{x}^{\prime 2}\right\rangle_{\perp}$ & $\frac{\tilde{r}_{x}^{2}}{4}+\frac{\tilde{\varepsilon}_{\hat{x}}^{2}}{4 \tilde{r}_{\tilde{x}}^{2}}$ & $\frac{\tilde{r}_{b}^{2}}{4}+\frac{\tilde{\varepsilon}^{2}}{4 \tilde{r}_{b}^{2}}$ \\
\hline$\left\langle\tilde{y}^{\prime 2}\right\rangle_{\perp}$ & $\frac{\tilde{r}_{\tilde{y}}^{2}}{4}+\frac{\tilde{\varepsilon}_{\tilde{y}}^{2}}{4 \tilde{r}^{2}}$ & $\frac{\tilde{r}_{b}^{2}}{4}+\frac{\tilde{\varepsilon}^{2}}{4 \tilde{r}^{2}}$ \\
\hline$\left\langle\tilde{x} \tilde{x}^{\prime}\right\rangle_{\perp}$ & $\frac{\tilde{r}_{\tilde{r}} \tilde{r}_{\tilde{x}}^{\prime}}{4}$ & $\frac{\tilde{r}_{b} \tilde{r}_{b}}{4}$ \\
\hline$\left\langle\tilde{y} \tilde{y}^{\prime}\right\rangle_{\perp}$ & $\frac{\tilde{r}_{y} \tilde{r}_{y}^{\prime}}{4}$ & $\frac{\tilde{r}_{b} \tilde{r}_{b}^{\prime}}{4}$ \\
\hline$\left\langle\tilde{x} \tilde{y}^{\prime}-\tilde{y} \tilde{x}^{\prime}\right\rangle_{\perp}$ & $\stackrel{4}{0}$ & $\stackrel{4}{P_{\theta}}$ \\
\hline $16\left[\left\langle\tilde{x}^{2}\right\rangle_{\perp}\left\langle\tilde{x}^{2}\right\rangle_{\perp}-\left\langle\tilde{x} \tilde{x}^{\prime}\right\rangle_{\perp}^{2}\right]$ & $\tilde{\varepsilon}_{\tilde{x}}^{2}$ & $\tilde{\varepsilon}^{2}$ \\
\hline $16\left[\left\langle\tilde{y}^{2}\right\rangle_{\perp}\left\langle\tilde{y}^{2}\right\rangle_{\perp}-\left\langle\tilde{y} \tilde{y}^{\prime}\right\rangle_{\perp}^{2}\right]$ & $\tilde{\varepsilon}_{\tilde{y}}^{2}$ & $\tilde{\varepsilon}^{2}$ \\
\hline
\end{tabular}

$$
\tilde{n}=\int d^{2} \tilde{x}^{\prime} \tilde{f}= \begin{cases}\frac{\lambda}{\pi \tilde{r}_{\tilde{x}} \tilde{r}_{\tilde{y}}}, & \text { if }\left(\tilde{x} / \tilde{r}_{\tilde{x}}\right)^{2}+\left(\tilde{y} / \tilde{r}_{\tilde{y}}\right)^{2}<1 \\ 0, & \text { otherwise }\end{cases}
$$

verifying consistency. Laboratory-frame statistical averages are simply expressed in terms of the rotating-frame distribution as

$$
\langle\cdots\rangle_{\perp}=\frac{\int d^{2} \tilde{x} \int d^{2} \tilde{x}^{\prime} \cdots \tilde{f}}{\int d^{2} \tilde{x} \int d^{2} \tilde{x}^{\prime} \tilde{f}} .
$$

Various moments of the Larmor-frame KV distribution (A9) are summarized in Table II. The moments in Table II can also be applied to the laboratory-frame KV distribution for quadrupole and continuous focusing channels by simply dropping all tildes. It should be stressed that the rotating-frame emittances $\tilde{\varepsilon}_{\tilde{x}}$ and $\tilde{\varepsilon}_{\tilde{y}}$ are not, in general, equal to the laboratory-frame emittances $\varepsilon_{x}$ and $\varepsilon_{y}$, which evolve in $s$ for an elliptical KV beam in a solenoidal focusing channel. Laboratory-frame projections of the moments are straightforward to calculate from the transformations (A3) and (A4).

The angular momentum of the KV distribution (A9) is important in understanding the structure of the equilibrium. The vector potential of the axial magnetic field can be expressed as $\mathbf{A}=\left(B_{z} / 2\right)(y \hat{\mathbf{x}}-x \hat{\mathbf{y}})$, and canonical momenta conjugate to the coordinates $Q_{x}=x$ and $Q_{y}=y$ are $P_{x}=x^{\prime}-k_{L} y$ and $P_{y}=y^{\prime}+k_{L} x$. Therefore, the single-particle canonical angular momentum $p_{\theta} \equiv$ $\mathbf{Q} \times \mathbf{P} \cdot \hat{\mathbf{z}}$ can be expressed as

$$
p_{\theta}=x y^{\prime}-y x^{\prime}+k_{L}\left(x^{2}+y^{2}\right)=\tilde{x} \tilde{y}^{\prime}-\tilde{y} \tilde{x}^{\prime} .
$$

For any distribution of particles, it follows from the equations of motion (A1) that the system canonical angular momentum

$$
P_{\theta} \equiv\left\langle p_{\theta}\right\rangle_{\perp}=\left\langle x y^{\prime}-y x^{\prime}\right\rangle_{\perp}+k_{L}\left\langle x^{2}+y^{2}\right\rangle_{\perp}
$$

satisfies

$$
\frac{d}{d s} P_{\theta}=\left(\kappa_{x}-\kappa_{y}\right)\langle x y\rangle_{\perp}-\frac{q}{m \gamma_{b}^{3} \beta_{b}^{2} c^{2}}\left\langle x \frac{\partial \phi}{\partial y}-y \frac{\partial \phi}{\partial x}\right\rangle_{\perp} .
$$

For a solenoidal focused beam, $\kappa_{x}=\kappa_{y}=\kappa$ and the first moment term in Eq. (A19) vanishes. Using Green's functions for the Poisson equation $\nabla^{2} \phi_{\perp}=-q n / \epsilon_{0}$, it can be shown that the second moment term in Eq. (A19) also vanishes provided any conducting boundaries are azimuthally invariant $(\partial / \partial \theta=0)$. Thus, in a solenoidal channel, $P_{\theta}=$ const for any distribution function focused within an axisymmetric beam pipe. For the specific KV distribution in Eq. (A9), we calculate

$$
P_{\theta} \equiv\left\langle p_{\theta}\right\rangle_{\perp}=\left\langle\tilde{x} \tilde{y}^{\prime}-\tilde{y} \tilde{x}^{\prime}\right\rangle_{\perp}=0
$$

This zero value of $P_{\theta}$ is appropriate for a beam launched from a field-free source (i.e., $B_{z}=0$ at the source location), and results in maximum focusability for given Larmor-frame emittances $\tilde{\varepsilon}_{\tilde{x}}$ and $\tilde{\varepsilon}_{\tilde{y}}$. Beams injected from a source immersed in a finite axial magnetic field $B_{z}$ have nonzero $P_{\theta}$ and higher effective emittances 
[34,35]. Also, for a KV equilibrium evolving in an alternating gradient focusing channel, Eq. (A9) can be applied in the laboratory frame (drop all tildes) to show that $P_{\theta}=$ $\left\langle x y^{\prime}-y x^{\prime}\right\rangle_{\perp}=0$ in this case too. Elliptical KV distributions analogous to Eq. (A9) can also be constructed for nonzero values of $P_{\theta}=$ const for both solenoidally focused beams in the Larmor-frame and quadrupole focused (possibly with skew couplings) beams in the laboratory frame. However, these distributions will result in envelope equations that differ in form from Eq. (4) and have more complicated invariant emittances explored by Dragt, Neri, and Rangarajan [36] and Barnard (see Ref. [37]). This follows in these situations because the elliptical beam axes rotate within the Larmor frame. Coupled moment formulations have been employed to analyze the special case of elliptical envelope perturbations about a round beam equilibrium for a continuously focused beam with $P_{\theta} \neq 0$ [38]. In the present paper, we explore only cases of envelope evolution that we can readily map to Eq. (4) while concretely connecting the model to physically relevant focusing systems and quantities. Elliptical beams with $P_{\theta} \neq 0$ in solenoidal or alternating gradient quadrupole focusing channels [39] are beyond the scope of the present analysis. For the special case of a round beam envelope (i.e., $\tilde{r}_{\tilde{x}}=\tilde{r}_{\tilde{y}} \equiv \tilde{r}_{b}$ ) with $P_{\theta} \neq 0$ in a solenoidal focusing channel, the envelope equations can also be mapped to the form of Eq. (A11) with a KV distribution previously analyzed by Chen, Pakter, and Davidson [40]. (In Ref. [40] the authors do not refer to the distribution analyzed as being $\mathrm{KV}$, but it is appropriate to label any distribution as being of KV form if it is a delta function of a Courant-Snyder invariant that is expressible as a quadratic form in canonically transformed phase-space variables and has a uniform density projection. An infinity of KV distributions can be constructed through linear canonical transformations of the quadratic standard form of the KV distribution [33].) For this special axisymmetric case the self-consistent $\mathrm{KV}$ distribution satisfying the Vlasov equation can be expressed as

$$
\begin{aligned}
\tilde{f}\left(\tilde{x}, \tilde{x}^{\prime}, \tilde{y}, \tilde{y}^{\prime}\right)=\frac{\lambda}{q \pi^{2} \tilde{\varepsilon}^{2}} \delta & {\left[\left(\frac{\tilde{x}}{\tilde{r}_{b}}\right)^{2}+\left(\frac{\tilde{r}_{b} \tilde{x}^{\prime}-\tilde{r}_{b}^{\prime} \tilde{x}}{\tilde{\varepsilon}}\right)^{2}+\left(\frac{\tilde{y}}{\tilde{r}_{b}}\right)^{2}\right.} \\
& +\left(\frac{\tilde{r}_{b} \tilde{y}^{\prime}-\tilde{r}_{b}^{\prime} \tilde{y}}{\tilde{\varepsilon}}\right)^{2}-\frac{4 P_{\theta}}{\tilde{\varepsilon}^{2}}\left(\tilde{x} \tilde{y}^{\prime}-\tilde{y} \tilde{x}^{\prime}\right) \\
& \left.-\left(1-\frac{4 P_{\theta}^{2}}{\tilde{\varepsilon}^{2}}\right)\right]
\end{aligned}
$$

and the corresponding emittances and envelope equation are given by Eqs. (A10) and (A11) with $\tilde{\varepsilon}_{\tilde{x}}=\tilde{\varepsilon}_{\tilde{y}} \equiv \tilde{\varepsilon}$ and $\tilde{r}_{\tilde{x}}=\tilde{r}_{\tilde{y}}=\tilde{r}_{b}$. The self-consistent density can be calculated from Eq. (A21) for $-1<2 P_{\theta} / \tilde{\varepsilon}<1$ (corresponding to sufficiently small angular momentum to be focusable) as

$$
\tilde{n}=\int d^{2} \tilde{x}^{\prime} \tilde{f}= \begin{cases}\frac{\lambda}{\pi \tilde{r}_{b}^{2}}, & \text { if }\left(\tilde{x} / \tilde{r}_{b}\right)^{2}+\left(\tilde{y} / \tilde{r}_{b}\right)^{2}<1, \\ 0, & \text { otherwise. }\end{cases}
$$

Additional moments are summarized in Table II for $-1<2 P_{\theta} / \tilde{\varepsilon}<1$. Note that nonzero $P_{\theta}$ results in directed azimuthal flow contributions to various moments. For an axisymmetric beam, it is instructive to employ Eqs. (A3) and (A7) to express the constant Larmor-frame emittance $\tilde{\varepsilon}$ in terms of laboratory-frame moments. This gives

$$
\tilde{\varepsilon}^{2}=\varepsilon_{x}^{2}-4\left\langle r^{2} \theta^{\prime}\right\rangle_{\perp}^{2}+4 P_{\theta}^{2}=\text { const, }
$$

where $\varepsilon_{x}=\varepsilon_{y}$ is the ordinary laboratory-frame emittance given by Eq. (5) and $r=\sqrt{x^{2}+y^{2}}$ and $\theta=$ $\tan ^{-1}(y, x)$ are the usual cylindrical particle coordinates in the laboratory frame. Because $P_{\theta}=$ const, it follows from Eq. (A23) that

$$
\varepsilon_{r} \equiv \sqrt{\varepsilon_{x}^{2}-4\left\langle r^{2} \theta^{\prime}\right\rangle_{\perp}^{2}}=\text { const }
$$

can be interpreted as a conserved radial emittance for an axisymmetric beam. In summary, round beam envelopes with $r_{x}=r_{y}$ and $P_{\theta} \neq 0$ can also be analyzed with the envelope equation (4) provided we interpret the results in the rotating Larmor frame and substitute

$$
\begin{aligned}
\kappa_{x} & =\kappa_{y} \rightarrow k_{L}^{2}, \\
r_{x} & =r_{y} \rightarrow \tilde{r}_{b}, \\
\varepsilon_{x} & =\varepsilon_{y} \rightarrow \tilde{\varepsilon}
\end{aligned}
$$

in Eq. (4). In this axisymmetric case, the initial phase of orientation of the stationary laboratory frame is arbitrary. Various authors have derived equivalent axisymmetric beam envelope equations valid for finite $P_{\theta}$ including Lee and Cooper [34], Miller [35], and Chen, Pakter and Davidson [40].

\section{APPENDIX B: NUMERICAL CALCULATION OF MATCHED BEAM CONDITIONS}

A variety of methods can be employed to calculate initial envelope conditions in $r_{j}\left(s_{i}\right)$ and $r_{j}^{\prime}\left(s_{i}\right)$ to satisfy the matching condition in Eq. (7) to adequate precision. Standard numerical methods to integrate nonlinear ordinary differential equations can be applied to Eq. (4) to calculate the trajectories $r_{j}(s)$ and $r_{j}^{\prime}(s)$ from initial values at $s=s_{i}$. These integrations define a nonlinear $4 \times 4$ transfer map $\mathbf{M}\left(s \mid s_{i}\right)$ as

$$
\mathbf{R}(s)=\mathbf{M}\left(s \mid s_{i}\right) \cdot \mathbf{R}\left(s_{i}\right),
$$

where

$$
\mathbf{R}(s)=\left(\begin{array}{c}
r_{x}(s) \\
r_{x}^{\prime}(s) \\
r_{y}(s) \\
r_{y}^{\prime}(s)
\end{array}\right)
$$


The nonlinear map $\mathbf{M}\left(s \mid s_{i}\right)$ depends on $\mathbf{R}\left(s_{i}\right)$. We have suppressed this dependence to avoid cumbersome notation. Using Eq. (B1), the matching condition in Eq. (7) can be expressed as

$$
\mathbf{R}\left(s_{i}\right)=\mathbf{M}\left(s_{i}+L_{p} \mid s_{i}\right) \cdot \mathbf{R}\left(s_{i}\right) .
$$

Many standard root finding procedures can be employed to iteratively improve a sufficiently close initial guess of the matching condition. One useful implementation for the matching iteration based on the multidimensional Newton-Ralphson formula with a transfer map [28] can be expressed as

$$
\begin{aligned}
\mathbf{R}_{k+1}\left(s_{i}\right)= & \mathbf{R}_{k}\left(s_{i}\right)-\left[\mathbf{I}-\mathbf{M}_{e}\left(s_{i}+L_{p} \mid s_{i}\right)\right]^{-1} \\
& \cdot\left[\mathbf{R}_{k}\left(s_{i}\right)-\mathbf{M}\left(s_{i}+L_{p} \mid s_{i}\right) \cdot \mathbf{R}_{k}\left(s_{i}\right)\right] .
\end{aligned}
$$

Here, $\mathbf{R}_{k}\left(s_{i}\right)$ denotes the matching conditions on the $k$ th iteration and $\mathbf{M}_{e}\left(s \mid s_{i}\right)$ is the transfer matrix defined by Eq. (46) with zero driving perturbations $\left(\delta \kappa_{j}=0, \delta Q=\right.$ 0 , and $\left.\delta \varepsilon_{j}=0\right)$ and $r_{j m}(s)$ and $r_{j m}^{\prime}(s)$ specified by $\mathbf{R}_{k}(s)$. Equation (B4) has been applied by Ryne [41] to calculate matching conditions and has the advantage of employing Jacobian elements $\mathbf{M}_{e}\left(s_{i}+L_{p} \mid s_{i}\right)$ that are calculated from a linear map of envelope perturbations about the trajectory $\mathbf{R}_{k}(s)$. This perturbation map is constructed from numerical solutions of ordinary differential equations rather than through finite differences of $\mathbf{M}$, which typically improves convergence properties relative to other standard root finding methods based only on evaluations of $\mathbf{M}$ or on finite differences of $\mathbf{M}$ that can degrade precision. To increase robustness, Eq. (B4) can be combined with root bracketing to bound iterations and other standard methods such as bisection if an iteration produces too large a deviation. The method is also useful to efficiently "polish" approximate matched envelope solutions to high accuracy.

The starting value $\mathbf{R}_{1}\left(s_{i}\right)$ of the matching iterations must lie within the "basin of attraction" of the desired solution under the method employed. This is particularly important when analyzing a wide variety of lattices parametrically. Robustness of the method associated with an estimate for $\mathbf{R}_{1}\left(s_{i}\right)$ lying sufficiently close to the desired solution will often be of greater practical concern than numerical efficiency. Adequate starting estimates can often be constructed from the continuous focusing model (see Sec. III) with $k_{\beta 0}=\sigma_{0} / L_{p}$. For lattices with few symmetries or high focusing strengths $\left(\max \left[\left|\kappa_{j}\right|\right]\right.$ large), better initial estimates for $\mathbf{R}_{1}\left(s_{i}\right)$ may be necessary. Any lattice symmetries reducing the dimension of the initial conditions should be exploited to speed up and simplify this procedure. Low-order terms in an iterative theory developed by Lee [25] can be straightforwardly applied to calculate good estimates for $\mathbf{R}_{1}\left(s_{i}\right)$. Although Lee considers only quadrupole focusing lattices, his methods are readily extendable to lattices with other simple symmetries. Alternatively, $\mathbf{R}_{1}\left(s_{i}\right)$ can be estimated by setting the perveance to $Q=0$ while keeping other beam and lattice parameters fixed to first analyze the envelope neglecting beam space charge. Then many established accelerator codes [42] can be applied to numerically calculate the socalled betatron function $\beta_{j}(s)$ (see Appendix E, $\beta_{j} \equiv w_{j}^{2}$ ) for the particle orbits in the $x$ and $y$ planes without space charge, and the $Q=0$ matched beam envelope is simply obtained from the betatron functions as $r_{j m}=\sqrt{\varepsilon_{j} \beta_{j}}$ [19]. The betatron functions can then be used to calculate trial $\mathbf{R}_{1}\left(s_{i}\right)$ to start an iterative procedure where the perveance is increased from zero to the desired value [43]. For each perveance iteration the matched beam initial conditions are calculated using numerical root finding.

\section{APPENDIX C: FRINGE FIELD EQUIVALENT MODELS FOR APPLIED FOCUSING ELEMENTS}

Physical electric or magnetic focusing elements in the laboratory have material structures that produce applied fields consistent with the three-dimensional Maxwell equations. The resulting fields will, in general, contain both linear components that contribute to the applied focusing functions $\kappa_{j}(s)$ (see Sec. II B) as well as nonlinear error terms outside the scope of this paper. Typically, the material extent of a focusing element is axially long relative to the transverse extent of its clearbore aperture. Near the axial midplane, the elements in this case can often be regarded as two-dimensional axially extruded structures, and the fields within this region can then be well approximated by lower-dimensional models. Near the ends, this lower-dimensional approximation breaks down, and there are significant field variations as well as unwanted nonlinear field terms. In many cases the ends have approximate left-right symmetries about the axial midplane of the element. Consistent with these field variations in the element, the linear focusing functions $\kappa_{j}(s)$ of the lattice will generally vary in $s$ : typically with near constant values in the neighborhood of the axial midplane till the end regions are approached, then smoothly dropping to exponentially small values outside of the ends. This $s$ variation near the ends is called the fringe field and is associated with all physical focusing elements, leading to the obvious question on how well so-called hard-edge lattice models with piecewise constant $\kappa_{j}(s)$ such as introduced in Sec. II B apply to physical lattices.

Differences between physical and hard-edge focusing functions $\kappa_{j}(s)$ can be regarded as driving perturbations [i.e., the $\delta \kappa_{j}(s)$ in Sec. II F] about a hard-edge lattice. Alternatively, the physical and hard-edge focusing functions can be thought of as producing different matched beam envelopes for the same beam parameters with deviations between these matched envelopes leading to possible variations in envelope mode structure about the matched beam. In either case, prescriptions must be given for the best possible choices of "equivalent" hard-edge focusing parameters. In this Appendix we take the latter 
interpretation of the physical and model (hard edge or otherwise) focusing functions giving different matched envelopes and develop procedures for fixing equivalent model focusing functions to the physical lattice that minimize deviations between physical and model matched beam envelopes. No fully general optimal procedure can be given, because deviations depend on the specific lattice considered and the structure of the matched envelope. With regards to low-order beam evolutions modeled by the KV envelope equation (4), we generally find that hard-edge models produce results that are close to physical models for a periodic lattice if appropriate, equivalent hard-edge parameters (e.g., for solenoids the occupancy $\eta$ and focusing strength $\hat{\kappa}$ ) are employed. The level of agreement improves for simpler lattices with higher degrees of symmetry (e.g., the solenoidal and quadrupole hard-edge lattices introduced in Sec. II B). This agreement is likely a consequence of compensating errors within a lattice period and that periodic focusing "kicks" in a repeating solution can be well modeled by kicks of varying shape that impart the same total impulse. On the other hand for more general lattices and evolutions (e.g., a matching section where beam envelope parameters are transformed and there is no periodicity), uncompensated accumulated phase errors in the envelope excursions can result in significant errors if accurate fringe field models are not employed.

No unique prescription exists on how to best fix parameters employed to model $\kappa_{j}(s)$ for either hard-edge models or for more detailed fringe field models. In both cases we refer best fit model parameters as "equivalent fringe" parameters. Here we outline several reasonable procedures that can be used to fix equivalent fringe parameters.

One way to fix equivalent fringe parameters is through the dynamics of the particles. Equating matrix elements of the $2 \times 2$ transfer maps $\mathbf{M}_{j}\left(s \mid s_{i}\right)$ [see Eq. (19)] connecting one side of the optic before the fringe entering the focusing element to the end of the fringe exiting the focusing element will result in equivalent evolutions in drift regions. It is usually sufficient to carry out such procedures only for the undepressed orbits ( $Q=0$ maps) which provide a direct measure of the applied focusing functions. This will result in three, rather than four, independent constraints in each of the $x$ and $y$ planes (the $x$ and $y$ planes can also yield identical or related constraints for some optics) because $\mathbf{M}_{j}\left(s \mid s_{i}\right)$ is symplectic and has a unit determinant. Unfortunately, these kinematic methods of fixing equivalent fringe parameters prove less than satisfactory in practical applications because they do not result in scale invariant models and must be repeated with changes in particle energy. Moreover, if there are more equivalent fringe parameters than kinematic constraints employed, the method is ambiguous and must be supplemented by additional constraints.
Because of these limitations, we employ an alternative, nonkinematic method of fixing equivalent fringe field parameters defined as follows. Denote the physical and equivalent fringe model focusing functions by $\kappa_{j}(s)$ and $\kappa_{f j}(s)$. Then we specify $\kappa_{f j}(s)$ by requiring the following:

(1) Equal focusing strength at the midplanes of the elements, i.e.,

$$
\kappa_{f j}\left(s_{m}\right)=\kappa_{j}\left(s_{m}\right),
$$

where $s=\left\{s_{m}\right\}$ are the axial coordinates of the midplanes of the physical focusing elements.

(2) Correct integrated focusing strength in each focusing element, i.e.,

$$
\int_{\text {element }} d s \kappa_{j}(s)=\int_{\text {element }} d s \kappa_{f j}(s),
$$

where the integration range contains the full fringe field of the physical and equivalent elements.

(3) Least-square error between the physical and fringe equivalent lattices. Here, we take the error measure to be given by

$$
\text { error }=\int_{s_{i}}^{s_{i}+L_{p}} d s\left[\kappa_{j}(s)-\kappa_{f j}(s)\right]^{2} .
$$

The constraint (1) allows easy understanding of the overall "excitation" needed for the focusing element. The physical element focusing strength $\kappa_{j}\left(s_{m}\right)$ can also be replaced in (1) by lower-dimensional optical models (e.g., a 2D analytical quadrupole model) to allow analytical estimates of needed excitations. The constraint (2) maintains a good low-order equivalency of focusing strength. To obtain higher-precision equivalences, (2) can be replaced by a constraint for equal values of the single-particle phase advance $\sigma_{0 j}$ in the physical and equivalent lattices for partial kinematic equivalency. Finally, (3) constrains any additional parameters after (1) and (2) establish low-order equivalency. Note that $\kappa_{f j}(s)=\kappa_{j}(s)$ corresponds to zero error and an identical kinematic transfer matrix $\mathbf{M}_{j}\left(s \mid s_{i}\right)$ between all points in the lattice with or without space charge. Constraints (2) and (3) both recover the correct thin-lens limit. Additional criteria can also be added to the error function in (3) to further constrain the equivalent fringe model to minimize peak envelope excursions and other criteria [44]. For the special case of solenoidal and FODO quadrupole lattices with piecewise constant $\kappa_{j}(s)$ as defined in Sec. II B, only two equivalent lattice parameters need be set (e.g., $\hat{\kappa}$ and $\eta$ for the solenoidal lattice) for which the first two constraints are sufficient and the third error constraint is not needed.

We illustrate the results of this equivalent fringe procedure with the FODO electric quadrupole lattice of the High Current Transport Experiment (HCX) at the Lawrence Berkeley National Laboratory [30,31]. As shown in the cross section in Fig. 22(a), the quadrupoles are formed from left and right conducting end plates held 
a) HCX Electrostatic Quadrupole Geometry

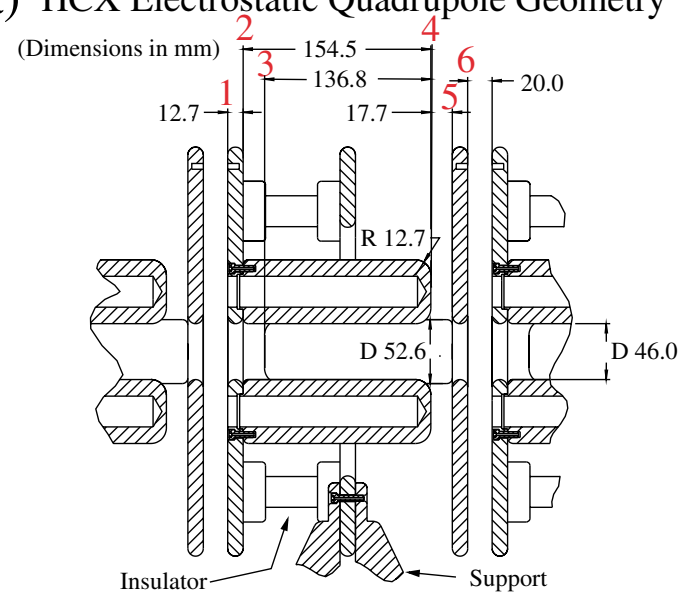

b) Physical and Square Edge Equivalent

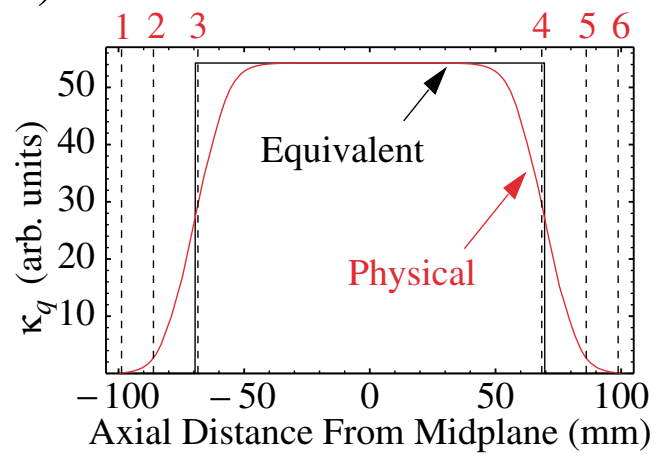

c) Physical and Ramped Edge Equivalent

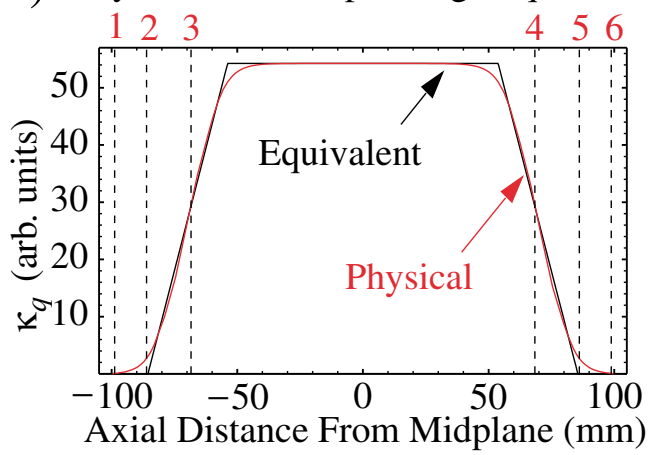

FIG. 22. (Color) Transverse cross section (a) of the electrostatic quadrupole lattice geometry of the HCX. In (b) and (c), corresponding focusing functions $\kappa_{q}(s)$ are plotted in arbitrary units versus axial distance from the axial midplane of the quadrupole. The physical lattice $\kappa_{q}(s)$ is shown (red) together with $\kappa_{q}(s)$ for equivalent fringe models with square and ramped edges (black) in (b) and (c). Dotted lines in (b) and (c) indicate axial locations of structures shown in (a) with labels $1-6$ in red.

at equal magnitude positive and negative biases and conducting rods attached to each end plate along the principal $x$ and $y$ axes as indicated. The structure is mechanically supported at the axial midplane by a plate connected to the end plates by insulators: end plates are $12.7 \mathrm{~mm}$ thick with clear-bore aperture holes of radii $r_{p}=23.0 \mathrm{~mm}$, rods are of radii $(8 / 7) r_{p}=26.3 \mathrm{~mm}$ (ratio chosen to minimize nonlinear fields in $2 \mathrm{D}$ axial extruded geometry of the midplane) and axial length $154.5 \mathrm{~mm}$, and gaps between rods and end plates are $17.7 \mathrm{~mm}$. The quadrupoles are arraigned with an overall lattice period of $L_{p}=435.2 \mathrm{~mm}$ and adjacent end plates are biased to the same potential. This detailed FODO lattice structure was loaded into a 3D electrostatic field solver in the WARP code [45] and the linear applied field moments were extracted from the full 3D electric field solution. These applied field moments can then be linearly scaled for the quadrupole bias (excitation) employed and then Eq. (11) is used to calculate the physical lattice focusing functions $\kappa_{x}(s)=-\kappa_{y}(s) \equiv \kappa_{q}(s)$ shown in Fig. 22. Two equivalent fringe focusing functions derived by the procedure above are also shown in Fig. 22 superimposed with the physical focusing function. The equivalent model in Fig. 22(b) is the hard-edge model in Sec. II B with square edges [i.e., piecewise constant $\kappa_{q}(s)$ ], and the equivalent model in Fig. 22(c) has $\kappa_{q}(s)$ with a constant value near the midplane and linear ramp variations in $s$ (zero to midplane value) entering and exiting the quadrupole. Only one quadrupole of the symmetric FODO lattice is shown for clarity. The procedure obtains for the square-edge model a best fit occupancy of $\eta=0.6949$ (corresponding to axial length $\eta L_{p} / 2=151.5 \mathrm{~mm}$ ), and for the ramped-edge model a central flat region of axial length $117.1 \mathrm{~mm}$ with ramped ends of length $34.5 \mathrm{~mm}$. The total error according to criteria (3) above is about 74 times larger for the square-edge model than for the ramped-edge model.

Matched beam envelopes for both the physical focusing function and the square-edge and ramped-edge equivalent fringe models shown in Fig. 22 are contrasted in Fig. 23. In this procedure the physical quadrupole excitation was chosen such that $\sigma_{0}=80^{\circ}$. For both solutions values of $\sigma_{0}$ and $\sigma / \sigma_{0}$ are listed for the same values of perveance and emittance corresponding to a usual operating point in the HCX experiment. Little difference in matched envelopes is observed between the physical and either equivalent fringe models with the linear ramped equivalent model giving smaller deviations than the square-edge model (matched envelopes almost overlay). Matched envelopes of physical and equivalent lattices deviate even less than those shown in Fig. 23 if the lattices are tuned independently to obtain the same value of $\sigma_{0}$ after the equivalent lattice is chosen as described above. These results illustrate the general feature that simple equivalent fringe models result in little change in the structure of the matched beam envelope for lattices with a high degree of symmetry.

\section{APPENDIX D: DERIVATION OF EQ. (18)}

The free-drift differential equation $r_{+}^{\prime \prime}=Q / r_{+}$ [Eq. (17)] can be integrated to give 
a)

b)

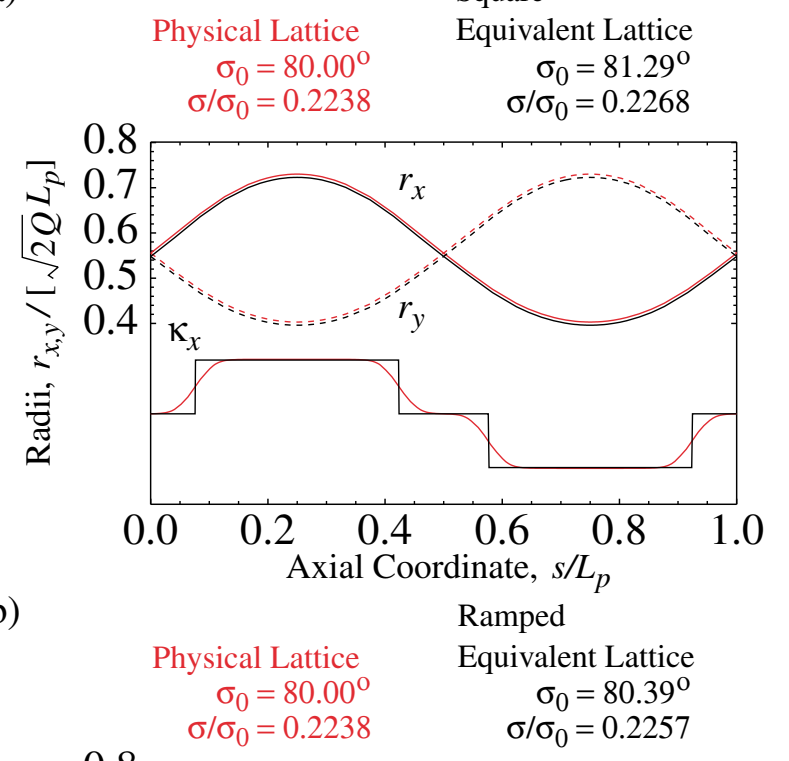

FIG. 23. (Color) Comparison of matched beam envelopes calculated from a full fringe field model (red) and an equivalent fringe model (black) for (a) the square-edge and (b) the ramped-edge models shown in Fig. 22. The presentation format of the matched solutions is the same as in Fig. 15. Both the physical and equivalent fringe model lattices have the same quadrupole excitation, beam parameters are $Q=8 . \times 10^{-4}$ and $\varepsilon_{x}=\varepsilon_{y}=60 \mathrm{mmmrad}$, and the undepressed and depressed single-particle phase advances $\sigma_{0}$ and $\sigma$ are listed above the plots.

$$
C_{1}=\frac{r_{+}^{\prime 2}}{2}-Q \ln r_{+}
$$

where $C_{1}=$ const is the scaled envelope Hamiltonian. We temporarily assume that $r_{+}^{\prime} \geq 0$. Then $r_{+}^{\prime}=$ $\sqrt{2 Q \ln r_{+}+2 C_{1}}$, and integrating once again we have

$$
s=e^{-C_{1}} \sqrt{\pi} \operatorname{erfi}\left[\sqrt{\ln \left(\frac{r_{+}}{\sqrt{2 Q}}\right)+C_{1}}\right]-C_{2},
$$

where $\quad C_{2}=$ const $\quad$ and $\quad \operatorname{erfi}(z)=\operatorname{erf}(i z) / i=$ $(2 / \sqrt{\pi}) \int_{0}^{z} d t \exp \left(t^{2}\right)$ is the imaginary error function. Evaluating Eqs. (D1) and (D2) at the initial conditions $r_{+}\left(s_{i}\right)$ and $r_{+}^{\prime}\left(s_{i}\right)$ at $s=s_{i}$ and using these results in

Eq. (D2) to eliminate $C_{1}$ and $C_{2}$ yields

$$
\begin{aligned}
\ln \left[\frac{r_{+}(s)}{r_{+}\left(s_{i}\right)}\right]= & -\frac{r_{+}^{\prime 2}\left(s_{i}\right)}{2 Q}+\left[\operatorname { e r f i } ^ { - 1 } \left\{\left[\frac{r_{+}^{\prime}\left(s_{i}\right)}{\sqrt{2 Q}}\right]\right.\right. \\
& \left.\left.+\sqrt{\frac{2 Q}{\pi}} e^{r_{+}^{\prime 2}\left(s_{i}\right) /(2 Q)} \frac{\left(s-s_{i}\right)}{r_{+}\left(s_{i}\right)}\right\}\right]^{2} .
\end{aligned}
$$

Because $r_{+}^{\prime \prime}=Q / r_{+}, \quad r_{+}$is an analytic function. Therefore, even though Eq. (D3) was derived under the assumption that $r_{+}^{\prime} \geq 0$, Eq. (D3) is also valid for $r_{+}^{\prime}<0$. Solving Eq. (D3) for $r_{+}(s)$ yields the free-drift solution given in Eq. (18).

\section{APPENDIX E: PHASE ADVANCE OF ENVELOPE MODES IN THE ZERO SPACE-CHARGE LIMIT}

In the limit of zero space charge $(Q=0)$, the equations of motion [see Eq. (1)] for the particle $x$ and $y$ orbits are decoupled and of the same form. Likewise, the resulting $x$ - and $y$-envelope equations [see Eq. (4)] are decoupled and of the same form. Therefore, we analyze only the $x$ equation here. The $x$ equation of motion of the particle is given by

$$
x^{\prime \prime}+\kappa_{x} x=0 .
$$

Here, $\kappa_{x}\left(s+L_{p}\right)=\kappa_{x}(s)$ describes the linear applied focusing function of a lattice with periodicity $L_{p}$ that is of otherwise unspecified form. To define the phase advance of the $x$ orbit, without loss in generality, we express the orbit in phase-amplitude form as $[18,19]$

$$
x(s)=A_{x} w_{x}(s) \cos \left[\psi_{x}(s)\right] .
$$

Here, $A_{x}=$ const, $\psi_{x}^{\prime}(s)=1 / w_{x}^{2}(s)$, and $w_{x}(s)$ is the positive solution to the equation

$$
w_{x}^{\prime \prime}+\kappa_{x} w_{x}-\frac{1}{w_{x}^{3}}=0
$$

satisfying the periodicity requirement $w_{x}\left(s+L_{p}\right)=$ $w_{x}(s)$. This equation is recognized as the scaled envelope equation with zero space charge [see Eq. (4)] and therefore the matched beam envelope $r_{x m}$ is related to the amplitude function $w_{x}$ as

$$
r_{x m}=\sqrt{\epsilon_{x}} w_{x} .
$$

Consistent with Eqs. (24) and (26), the single-particle phase advance $\sigma_{0 x}$ of oscillations in $x$ over one lattice period is

$$
\sigma_{0 x}=\psi_{x}\left(s_{i}+L_{p}\right)-\psi_{x}\left(s_{i}\right)=\int_{s_{i}}^{s_{i}+L_{p}} \frac{d s}{w_{x}^{2}},
$$

independent of the initial axial coordinate $s_{i}$.

Consistent with the $x$-particle equation of motion (E1), the beam envelope equation for $r_{x}=2 \sqrt{\left\langle x^{2}\right\rangle_{\perp}}$ is

$$
r_{x}^{\prime \prime}+\kappa_{x} r_{x}-\frac{\epsilon_{x}^{2}}{r_{x}^{3}}=0 .
$$


We expand $r_{x}$ as $r_{x}=r_{x m}+\delta r_{x}$ where $r_{x m}=\sqrt{\epsilon_{x}} w_{x}$ is the matched beam radius, and $\delta r_{x}$ is a small-amplitude perturbation about the matched beam. Then $\delta r_{x}$ satisfies the linearized envelope equation [see also Eq. (37)]

$$
\delta r_{x}^{\prime \prime}+\kappa_{x} \delta r_{x}+3 \frac{\epsilon_{x}^{2}}{r_{x m}^{4}} \delta r_{x}=0 .
$$

Paralleling the analysis above for the particle orbits, the solution to the linearized envelope equation can be expressed in phase-amplitude form as

$$
\delta r_{x}(s)=A_{e} w_{e}(s) \cos \left[\psi_{e}(s)\right] .
$$

Here, $A_{e}=$ const, $\psi_{e}^{\prime}(s)=1 / w_{e}^{2}(s)$, and $w_{e}(s)$ the positive solution to

$$
w_{e}^{\prime \prime}+\kappa_{x} w_{e}+\frac{3}{w_{x}^{4}} w_{e}-\frac{1}{w_{e}^{3}}=0
$$

satisfying the periodicity requirement $w_{e}\left(s+L_{p}\right)=$ $w_{e}(s)$. The phase advance $\sigma_{e x}$ of oscillations in $\delta r_{x}$ over one lattice period is given by

$$
\sigma_{e x}=\psi_{e}\left(s_{i}+L_{p}\right)-\psi_{e}\left(s_{i}\right)=\int_{s_{i}}^{s_{i}+L_{p}} \frac{d s}{w_{e}^{2}} .
$$

Comparison of Eqs. (E3) and (E9) shows that the solution for $w_{e}$ is connected to $w_{x}$ as

$$
w_{e}=\frac{w_{x}}{\sqrt{2}} .
$$

Thus, from Eqs. (E5) and (E10), we have

$$
\sigma_{e x}=2 \sigma_{0 x} .
$$

Equation (E12) proves that in the limit of zero space charge the phase advance of envelope oscillations in $\delta r_{x}$ is 2 times the phase advance of undepressed particle oscillations in the $x$ particle orbit. An analogous result to Eq. (E12) holds in the $y$ plane. Equation (E12) is straightforward to generalize to show that for zero space charge and focusing lattices with arbitrary (possibly nonperiodic) $\kappa_{x}(s)$ that the rate of phase accumulation of a small-amplitude envelope perturbation about a (possibly nonperiodic) reference envelope evolution is twice the rate of phase accumulation of the single-particle orbit.

\section{APPENDIX F: ENVELOPE MODE COURANT-SNYDER INVARIANTS}

For continuous focusing or solenoidal transport channels with $\kappa_{x}=\kappa_{y} \equiv \kappa$, undriven envelope perturbations $\delta r_{ \pm}=\left(r_{x} \pm r_{y}\right) / 2$ satisfy the uncoupled equations [see Eq. (45)]

$$
\begin{aligned}
& \delta r_{+}^{\prime \prime}+\kappa \delta r_{+}+\frac{2 Q}{r_{m}^{2}} \delta r_{+}+\frac{3 \varepsilon^{2}}{r_{m}^{4}} \delta r_{+}=0 \\
& \delta r_{-}^{\prime \prime}+\kappa \delta r_{-}+\frac{3 \varepsilon^{2}}{r_{m}^{4}} \delta r_{-}=0
\end{aligned}
$$

Here, $\kappa\left(s+L_{p}\right)=\kappa(s)$ describes the linear applied focusing forces of the lattice with periodicity $L_{p}$ and is otherwise unspecified $\left(\kappa=k_{\beta 0}^{2}=\right.$ const for continuous focusing). As in standard treatments of single-particle orbits $[18,19]$, without loss in generality, we express the envelope perturbations in phase-amplitude form as

$$
\delta r_{ \pm}=A_{ \pm} w_{ \pm}(s) \cos \left[\psi_{ \pm}(s)\right] .
$$

Here, $A_{ \pm}=$const, $\psi_{ \pm}^{\prime}=1 / w_{ \pm}^{2}$, and $w_{ \pm}$are positive solutions to the equations

$$
\begin{aligned}
& w_{+}^{\prime \prime}+\kappa w_{+}+\frac{2 Q}{r_{m}^{2}} w_{+}+\frac{3 \varepsilon^{2}}{r_{m}^{4}} w_{+}-\frac{1}{w_{+}^{3}}=0, \\
& w_{-}^{\prime \prime}+\kappa w_{-}+\frac{3 \varepsilon^{2}}{r_{m}^{4}} w_{-}-\frac{1}{w_{-}^{3}}=0
\end{aligned}
$$

satisfying the periodicity requirements $w_{ \pm}\left(s+L_{p}\right)=$ $w_{ \pm}(s)$. Employing scaling procedures analogous to those presented in Sec. IIE, it can be shown that the envelope amplitude functions $w_{ \pm}$can be regarded as being parametrized by $L_{p}, \sigma_{0}, \sigma / \sigma_{0}$, and parameters that describe the shape of the lattice focusing function $\kappa(s)$. Note that in contrast to the usual single-particle amplitude functions of undepressed particle orbits $[18,19], w_{ \pm}$will also depend on the matched beam space-charge depression parameter $\sigma / \sigma_{0}$ in addition to the lattice parameters $\sigma_{0}, L_{p}$, etc. The phase advance $\sigma_{ \pm}$of oscillations in $\delta r_{ \pm}$over one lattice period is given by

$$
\sigma_{ \pm}=\psi_{ \pm}\left(s_{i}+L_{p}\right)-\psi_{ \pm}\left(s_{i}\right)=\int_{s_{i}}^{s_{i}+L_{p}} \frac{d s}{w_{ \pm}^{2}},
$$

independent of the initial coordinate axial $s_{i}$. Differentiating Eq. (F2) and employing $\psi_{ \pm}^{\prime}=1 / w_{ \pm}^{2}$ to simplify the result gives

$$
\begin{aligned}
\frac{\delta r_{ \pm}}{w_{ \pm}} & =A_{ \pm} \cos \psi_{ \pm}, \\
w_{ \pm}^{\prime} \delta r_{ \pm}-w_{ \pm} \delta r_{ \pm}^{\prime} & =A_{ \pm} \sin \psi_{ \pm} .
\end{aligned}
$$

Squaring and adding these equations, we obtain quadratic Courant-Snyder invariants given by

$$
\left(\frac{\delta r_{ \pm}}{w_{ \pm}}\right)^{2}+\left(w_{ \pm}^{\prime} \delta r_{ \pm}-w_{ \pm} \delta r_{ \pm}^{\prime}\right)^{2}=A_{ \pm}^{2}=\text { const. }
$$

These invariants of the envelope perturbations are equations of ellipses in $\delta r_{ \pm}-\delta r_{ \pm}^{\prime}$ phase space where the axes of the ellipses do not, in general, align with the $\delta r_{ \pm}$and $\delta r_{ \pm}^{\prime}$ axes. They can be interpreted as area measures because the set of all initial conditions satisfying Eq. (F6) (corresponding to all pure modes with the same oscillation amplitude) will correspond to perturbations that evolve to trace out an ellipse in $\delta r_{ \pm}-\delta r_{ \pm}^{\prime}$ phase space with area $\pi A_{ \pm}=$const. Analysis of the evolution of this constant area ellipse (in terms of elongation and rotation) can be facilitated by introducing Twiss parameters as in standard treatments of uncoupled single-particle dynamics 
[19]. It is straightforward to show that Courant-Snyder invariants directly analogous to those in Eq. (F6) apply to nonperiodic lattices with general $\kappa(s)$ for any small-amplitude envelope perturbations about a reference envelope evolution that need not be matched. In this nonperiodic case, $w_{ \pm}$satisfies Eq. (F3) for a general reference envelope solution $r_{m}$ and $w_{ \pm}$need not be periodic.

Unfortunately, the analysis of Courant-Snyder invariants for the more general case of undriven coupled envelope modes evolving according to [see Eq. (37)]

$$
\delta r_{j}^{\prime \prime}+\kappa_{j} \delta r_{j}+\frac{2 Q}{\left(r_{x m}+r_{y m}\right)^{2}}\left(\delta r_{x}+\delta r_{y}\right)+\frac{3 \varepsilon_{j}^{2}}{r_{j m}^{4}} \delta r_{j}=0
$$

with $j=x, y$, and $\kappa_{x} \neq \kappa_{y}$ is considerably more complicated than in the continuous or solenoidal focusing case analyzed above. This complication arises because when $\kappa_{x} \neq \kappa_{y}$ the normal coordinates of the coupled modes are not simply expressible. In the previous case, the decoupled sum and difference coordinates $\delta r_{ \pm}=$ $\left(r_{x} \pm r_{y}\right) / 2$ provided simple expressions of the normal coordinates. For $\kappa_{x} \neq \kappa_{y}$, the normal coordinates are expressible in terms of the $s$-varying eigenvectors of the transfer map given by Eq. (47). Fortunately, Edwards and Teng [46] have presented a general formulation for the analysis of two-dimensional coupled linear motion that can be directly applied to coupled envelope modes. To connect this study to the present situation, the equations of motion (F7) of the envelope perturbations can be expressed in Hamiltonian form as

$$
\frac{d}{d s} \delta r_{j}=\frac{\partial H}{\partial \delta r_{j}^{\prime}}, \quad \frac{d}{d s} \delta r_{j}^{\prime}=-\frac{\partial H}{\partial \delta r_{j}},
$$

where

$$
\begin{aligned}
H= & \frac{1}{2}\left(\delta r_{x}^{\prime 2}+\delta r_{y}^{\prime 2}\right)+\frac{1}{2} \kappa_{x} \delta r_{x}^{2}+\frac{1}{2} \kappa_{y} \delta r_{y}^{2} \\
& +\frac{Q}{\left(r_{x m}+r_{y m}\right)^{2}}\left(\delta r_{x}+\delta r_{y}\right)^{2}+\frac{3}{2} \frac{\varepsilon_{x}^{2}}{r_{x m}^{4}} \delta r_{x}^{2}+\frac{3}{2} \frac{\varepsilon_{y}^{2}}{r_{y m}^{4}} \delta r_{y}^{2}
\end{aligned}
$$

is the envelope Hamiltonian. From this Hamiltonian formulation, the correspondence of variables and coefficients employed by Edwards and Teng to those used here are readily identified. The conjugate variables $x, p_{x}$ and $y, p_{y}$ of Edwards and Teng are replaced by

$$
\begin{aligned}
x & \rightarrow \delta r_{x}, \\
p_{x} & \rightarrow \delta r_{x}^{\prime}, \\
y & \rightarrow \delta r_{y}, \\
p_{y} & \rightarrow \delta r_{y}^{\prime},
\end{aligned}
$$

and the coefficients $L, F, G$, and $K$ are identified as

$$
\begin{aligned}
& L=0, \\
& F=\kappa_{x}+\frac{2 Q}{\left(r_{x m}+r_{y m}\right)^{2}}, \\
& G=\kappa_{y}+\frac{2 Q}{\left(r_{x m}+r_{y m}\right)^{2}}, \\
& K=\frac{2 Q}{\left(r_{x m}+r_{y m}\right)^{2}} .
\end{aligned}
$$

The quadratic invariants calculated by Edwards and Teng can then be directly applied and are generalized Courant-Snyder invariants of the coupled envelope modes. Application and interpretation of these coupled motion invariants are considerably more complicated than in the case presented above for solenoidal and continuous focusing. However, the structure and consequences of the generalized invariants are loosely analogous to the situation for the uncoupled motion CourantSnyder invariants.

\section{APPENDIX G: MATRIX ANALYSIS OF CONTINUOUS FOCUSING NORMAL ENVELOPE MODES}

For continuous focusing, the matrix form of the equation of motion of undriven envelope perturbations $\delta \mathbf{R}=$ $\left(\delta r_{x}, \delta r_{x}^{\prime}, \delta r_{y}, \delta r_{y}^{\prime}\right)$ is given by $d \delta \mathbf{R} / d s+\mathbf{K} \cdot \delta \mathbf{R}=0$ [Eq. (38) with $\delta \mathbf{P}=0$ ] with a constant coupling matrix $\mathbf{K}$ given by Eq. (40). The equilibrium constraint equation (61) can be used to evaluate $\mathbf{K}$ as

$$
\mathbf{K}=\left[\begin{array}{cccc}
0 & -1 & 0 & 0 \\
\frac{\sigma_{+}^{2}+\sigma_{-}^{2}}{2 L_{p}^{2}} & 0 & \frac{\sigma_{+}^{2}-\sigma_{-}^{2}}{2 L_{p}^{2}} & 0 \\
0 & 0 & 0 & -1 \\
\frac{\sigma_{+}^{2}-\sigma_{-}^{2}}{2 L_{p}^{2}} & 0 & \frac{\sigma_{+}^{2}+\sigma_{-}^{2}}{2 L_{p}^{2}} & 0
\end{array}\right] .
$$

Here we have expressed the elements of $\mathbf{K}$ in terms of the phase advances of the breathing and quadrupole modes $\sigma_{+}=\sqrt{2 \sigma_{0}^{2}+2 \sigma^{2}}$ and $\sigma_{-}=\sqrt{\sigma_{0}^{2}+3 \sigma^{2}}$ as defined in Eq. (64). The equation of motion for $\delta \mathbf{R}$ is trivially integrated from an initial condition $\delta \mathbf{R}\left(s_{i}\right)$ at axial coordinate $s=s_{i}$ as

$$
\delta \mathbf{R}(s)=\mathbf{M}_{e}\left(s \mid s_{i}\right) \cdot \delta \mathbf{R}\left(s_{i}\right),
$$

where

$$
\mathbf{M}_{e}\left(s \mid s_{i}\right)=e^{-\mathbf{K}\left(s-s_{i}\right)}
$$

is the transfer matrix of the envelope perturbations. This solution is equivalent to the one presented in Eq. (65).

Any linear transform of the envelope coordinates $\delta \mathbf{R}$ can be expressed as

$$
\delta \tilde{\mathbf{R}}=\mathbf{T} \cdot \delta \mathbf{R},
$$

where $\mathbf{T}=$ const is some invertible $4 \times 4$ matrix and $\delta \tilde{\mathbf{R}}$ is the transformed (tilde variables) envelope coordinate vector. The equation of motion in transformed coordinates becomes 


$$
\frac{d}{d s} \delta \tilde{\mathbf{R}}+\tilde{\mathbf{K}} \cdot \delta \tilde{\mathbf{R}}=0
$$

where

$$
\tilde{\mathbf{K}}=\mathbf{T} \cdot \mathbf{K} \cdot \mathbf{T}^{-1} .
$$

The solution to Eq. (G5) is given by

$$
\delta \tilde{\mathbf{R}}(s)=\tilde{\mathbf{M}}_{e}\left(s \mid s_{i}\right) \cdot \delta \tilde{\mathbf{R}}\left(s_{i}\right)
$$

where the transformed transfer map

$$
\tilde{\mathbf{M}}_{e}\left(s \mid s_{i}\right)=e^{-\tilde{\mathbf{K}}\left(s-s_{i}\right)}
$$

is related to the untransformed map $\mathbf{M}_{e}\left(s \mid s_{i}\right)$ by

$$
\tilde{\mathbf{M}}_{e}\left(s \mid s_{i}\right)=\mathbf{T} \cdot \mathbf{M}_{e}\left(s \mid s_{i}\right) \cdot \mathbf{T}^{-1} .
$$

The matrix $\mathbf{M}_{e}\left(s_{i}+L_{p} \mid s_{i}\right)$ has four eigenvalues $\lambda_{n}$ with $n=1,2,4$, and 4 defined by $\mathbf{M}_{e}\left(s_{i}+L_{p}\right)$ $\left.s_{i}\right) \cdot \mathbf{E}_{n}=\lambda_{n} \mathbf{E}_{n}$ [see Eq. (47)]. Transforming this equation gives $\tilde{\mathbf{M}}_{e}\left(s_{i}+L_{p} \mid s_{i}\right) \cdot \tilde{\mathbf{E}}_{n}=\lambda_{n} \tilde{\mathbf{E}}_{n}$, where $\tilde{\mathbf{E}}_{n}=\mathbf{T} \cdot$
$\mathbf{E}_{n}$, thereby showing that the eigenvalues of $\mathbf{M}_{e}$ are invariant under the transform T. Similarly, the four eigenvalues of the matrix $\mathbf{K}$ are equal to the eigenvalues of the transformed matrix $\tilde{\mathbf{K}}=\mathbf{T} \cdot \mathbf{K} \cdot \mathbf{T}^{-1}$. Consider a transform $\mathbf{T}$ to fully decoupled coordinates $\delta \tilde{\mathbf{R}}$ where $\tilde{\mathbf{M}}_{e}\left(s \mid s_{i}\right)$ is diagonal. Then from Eq. (G8) $\tilde{\mathbf{K}}$ is also diagonal. In this representation, the diagonal elements of both $\tilde{\mathbf{M}}_{e}\left(s_{i}+L_{p} \mid s_{i}\right)$ and $\tilde{\mathbf{K}}$ are their respective eigenvalues. The eigenvalues of $\mathbf{K}$ can be calculated using Eq. (G1) as $\pm i \sigma_{+} / L_{p}$ and $\pm i \sigma_{-} / L_{p}$. Therefore,

$$
\tilde{\mathbf{K}}=\mathbf{T} \cdot \mathbf{K} \cdot \mathbf{T}^{-1}=\left[\begin{array}{cccc}
-i \frac{\sigma_{+}}{L_{p}} & 0 & 0 & 0 \\
0 & i \frac{\sigma_{+}}{L_{p}} & 0 & 0 \\
0 & 0 & -i \frac{\sigma_{-}}{L_{p}} & 0 \\
0 & 0 & 0 & i \frac{\sigma_{-}}{L_{p}}
\end{array}\right]
$$

and Eq. (G8) gives

$$
\tilde{\mathbf{M}}_{e}\left(s \mid s_{i}\right)=\left[\begin{array}{cccc}
\exp \left(i \sigma_{+} \frac{s-s_{i}}{L_{p}}\right) & 0 & 0 & 0 \\
0 & \exp \left(-i \sigma_{+} \frac{s-s_{i}}{L_{p}}\right) & 0 & 0 \\
0 & 0 & \exp \left(i \sigma_{-} \frac{s-s_{i}}{L_{p}}\right) & 0 \\
0 & 0 & 0 & \exp \left(-i \sigma_{-} \frac{s-s_{i}}{L_{p}}\right)
\end{array}\right]
$$

From Eq. (G10) the eigenvalues of $\mathbf{M}_{e}\left(s_{i}+L_{p} \mid s_{i}\right)$ are identified as $\lambda_{n}=e^{ \pm i \sigma_{+}}, e^{ \pm i \sigma_{-}}$. To complete the analysis, the fully decoupled solution for $\delta \tilde{\mathbf{R}}(s)$ given by Eqs. (G7) and (G10) is connected to the untransformed coordinates $\delta \mathbf{R}=$ $\left(\delta r_{x}, \delta r_{x}^{\prime}, \delta r_{y}, \delta r_{y}^{\prime}\right)$ by $\delta \tilde{\mathbf{R}}=\mathbf{T} \cdot \delta \mathbf{R}$ through the transformation

$$
\mathbf{T}=\frac{1}{2}\left[\begin{array}{cccc}
-i \frac{\sigma_{+}}{L_{p}} & -1 & -i \frac{\sigma_{+}}{L_{p}} & -1 \\
-i \frac{\sigma_{+}}{L_{p}} & 1 & -i \frac{\sigma_{+}}{L_{p}} & 1 \\
-i \frac{\sigma_{-}}{L_{p}} & -1 & i \frac{\sigma_{-}}{L_{p}} & 1 \\
-i \frac{\sigma_{-}}{L_{p}} & 1 & i \frac{\sigma_{-}}{L_{p}} & -1
\end{array}\right], \quad \mathbf{T}^{-1}=\frac{1}{2}\left[\begin{array}{cccc}
i \frac{L_{p}}{\sigma_{+}} & i \frac{L_{p}}{\sigma_{+}} & i \frac{L_{p}}{\sigma_{-}} & i \frac{L_{p}}{\sigma_{-}} \\
i \frac{L_{p}}{\sigma_{+}} & i \frac{L_{p}}{\sigma_{+}} & -i \frac{L_{p}}{\sigma_{-}} & -i \frac{L_{p}}{\sigma_{-}} \\
-1 & 1 & 1 & -1
\end{array}\right]
$$

It is instructive to examine the connection of this matrix transformation approach to the decoupled sum and difference coordinates $\delta r_{ \pm}=\left(\delta r_{x} \pm \delta r_{y}\right) / 2 \mathrm{em}-$ ployed in Sec. III. Taking $\delta \tilde{\mathbf{R}}=\left(\delta r_{+}, \delta r_{+}^{\prime}, \delta r_{-}, \delta r_{-}^{\prime}\right)=$ $\mathbf{T} \cdot \delta \mathbf{R}$ corresponds to the linear transformation

$$
\mathbf{T}=\frac{1}{2} \mathbf{T}^{-1}=\frac{1}{2}\left[\begin{array}{cccc}
1 & 0 & 1 & 0 \\
0 & 1 & 0 & 1 \\
1 & 0 & -1 & 0 \\
0 & 1 & 0 & -1
\end{array}\right] .
$$

Under this transformation, $\tilde{\mathbf{K}}$ is block diagonal with

$$
\tilde{\mathbf{K}}=\mathbf{T} \cdot \mathbf{K} \cdot \mathbf{T}^{-1}=\left[\begin{array}{cccc}
0 & -1 & 0 & 0 \\
\frac{\sigma_{+}^{2}}{L_{p}^{2}} & 0 & 0 & 0 \\
0 & 0 & 0 & -1 \\
0 & 1 & \frac{\sigma_{2}^{2}}{L_{p}^{2}} & 0
\end{array}\right] .
$$

The corresponding transfer map $\tilde{\mathbf{M}}_{e}\left(s \mid s_{i}\right)=$ $\exp \left[-\tilde{\mathbf{K}}\left(s-s_{i}\right)\right]$ can be directly calculated using the Taylor series definition of the matrix exponential, recognizing the recursion among terms, and summing the resulting series to give

$$
\tilde{\mathbf{M}}_{e}\left(s \mid s_{i}\right)=\left[\begin{array}{cccc}
\cos \left(\sigma_{+} \frac{s-s_{i}}{L_{p}}\right) & \frac{L_{p}}{\sigma_{+}} \sin \left(\sigma_{+} \frac{s-s_{i}}{L_{p}}\right) & 0 & 0 \\
-\frac{\sigma_{+}}{L_{p}} \sin \left(\sigma_{+} \frac{s-s_{i}}{L_{p}}\right) & \cos \left(\sigma_{+} \frac{s-s_{i}}{L_{p}}\right) & 0 & 0 \\
0 & 0 & \cos \left(\sigma_{-} \frac{s-s_{i}}{L_{p}}\right) & \frac{L_{p}}{\sigma_{-}} \sin \left(\sigma_{-} \frac{s-s_{i}}{L_{p}}\right) \\
0 & 0 & -\frac{\sigma_{-}}{L_{p}} \sin \left(\sigma_{-} \frac{s-s_{i}}{L_{p}}\right) & \cos \left(\sigma_{-} \frac{s-s_{i}}{L_{p}}\right)
\end{array}\right] .
$$


The block diagonal form of Eq. (G13) corresponds to rotations in the decoupled $r_{+}-r_{+}^{\prime}$ and $r_{-}-r_{-}^{\prime}$ phase spaces. This solution also follows immediately from Eq. (65). Finally, it is instructive to point out that the transformation to fully decoupled coordinates presented in Eq. (G11) can be derived in terms of two successive linear transformations, first a transformation to decoupled $\delta r_{ \pm}$coordinates as given by Eq. (G12) and then a transformation from trigonometric forms (i.e., $\cos \left[\sigma_{ \pm}\left(s-s_{i}\right) / L_{p}\right]$ ) to exponential forms (i.e., $e^{\left.i \sigma_{ \pm}\left(s-s_{i}\right) / L_{p}\right)}$.

\section{APPENDIX H: THIN-LENS ANALYSIS OF NORMAL ENVELOPE MODES IN PERIODIC SOLENOIDAL AND FODO QUADRUPOLE FOCUSING CHANNELS AT ZERO AND FULL SPACE-CHARGE DEPRESSION}

Normal envelope modes of thin-lens $\left(\eta \rightarrow 0\right.$ with $\sigma_{0}$ fixed) solenoidal and FODO $(\alpha=1 / 2)$ quadrupole focusing channels can be analyzed analytically in the limit of zero $\left(\sigma \rightarrow \sigma_{0}\right)$ and full $(\sigma \rightarrow 0)$ space-charge depression [47]. In the zero space-charge limit, the $x$ - and $y$-envelope coordinates decouple and the general analysis in Sec. II F applies to show that all envelope modes have phase advance $2 \sigma_{0}$. As a consequence of this phase advance limit and the decoupling, instabilities are possible only for $\sigma_{0}=90^{\circ}$ and $180^{\circ}$. A straightforward thin-lens based transfer matrix analysis of single-particle orbits can then be used to show that there is no envelope instability for $\sigma_{0}=90^{\circ}$, and there is linear growth in oscillation amplitude with lattice periods traversed for $\sigma_{0}=180^{\circ}$.

To analyze the opposite limit of full space-charge depression, we proceed as follows. Let $L$ be the length of the free-drift interval between the two subsequent thin lenses, solenoids or quadrupoles $\left(L_{p}=L\right.$ for solenoids and $L_{p}=2 L$ for quadrupoles) as illustrated in Fig. 24 . By symmetry we need only consider the envelope evolution of the beam between two lenses. We take the first lens to be at axial location $s=-L / 2$ and the second one to be at $s=L / 2$.

The envelope equation (4) for the case of a fully depressed beam (with $\sigma=0$ ), which corresponds to zero beam emittance $\left(\varepsilon_{x}=\varepsilon_{y}=0\right)$, can be written in terms of scaled sum and difference coordinates $R_{ \pm}=\left(r_{x} \pm\right.$ $\left.r_{y}\right) /(2 \sqrt{2 Q})$ as

$$
\begin{aligned}
& 2 R_{+}^{\prime \prime}(s)+2 \kappa_{x}(s) R_{+}(s)-\frac{1}{R_{+}(s)}=0, \\
& 2 R_{-}^{\prime \prime}(s)+2 \kappa_{x}(s) R_{-}(s)=0
\end{aligned}
$$

for solenoidal focusing, and

$$
\begin{aligned}
& 2 R_{+}^{\prime \prime}(s)+2 \kappa_{x}(s) R_{-}(s)-\frac{1}{R_{+}(s)}=0, \\
& 2 R_{-}^{\prime \prime}(s)+2 \kappa_{x}(s) R_{+}(s)=0
\end{aligned}
$$

for quadrupole focusing. Here, we have employed $\kappa_{x}=$ $\kappa_{y}$ for solenoidal focusing and $\kappa_{x}=-\kappa_{y}$ for quadrupole focusing. For the solenoidal channel all thin-lens focus-
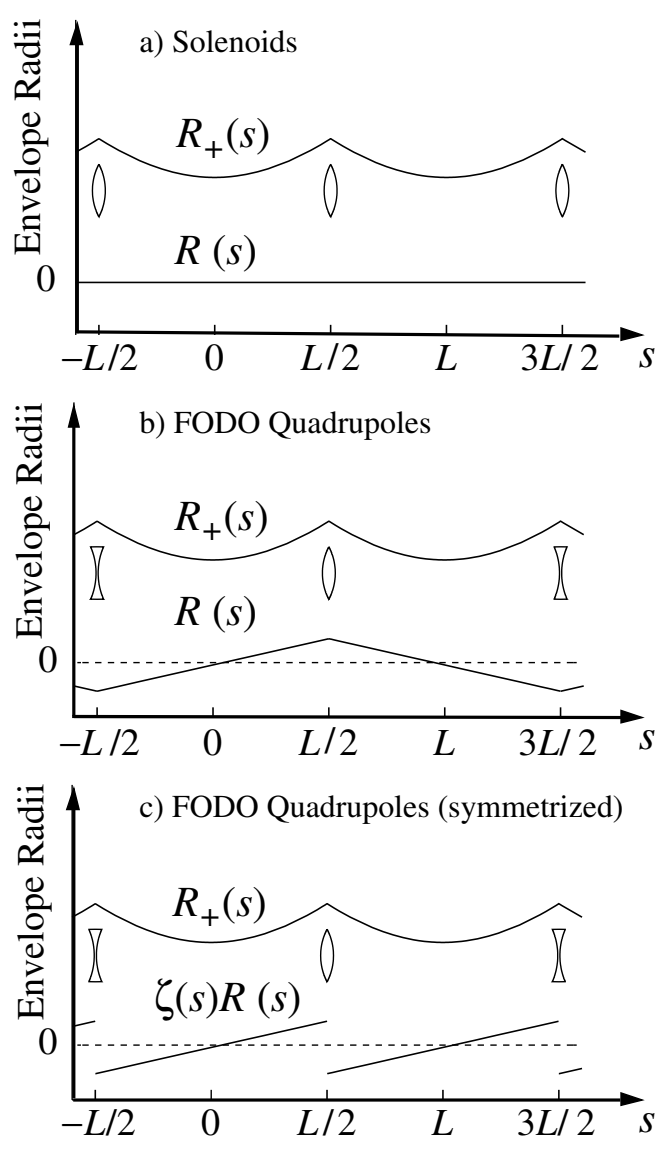

FIG. 24. Matched beam envelopes $R_{ \pm}(s)$ and transport lattice for (a) solenoid and (b) FODO quadrupole thin-lens transport channels. In (c), $\zeta(s) R_{-}(s)$ is plotted for the quadrupole channel.

ing kicks are focusing in $x$. Without loss of generality, we assume that in the alternating gradient channel that the lens at $s=L / 2$ is focusing in $x$. Then for both thin-lens solenoids and quadrupoles we take near $s=L / 2$ [see Eq. (13)]

$$
\kappa_{x}(s)=\frac{1}{f} \delta\left(s-\frac{L}{2}\right)
$$

where $f=$ const is the thin-lens focal length and $\delta(s)$ is the Dirac delta function. The focal length $f$ can be related to the undepressed particle phase advance over one lattice period $\sigma_{0}$ as [see Eq. (30)]

$$
\frac{L}{f}= \begin{cases}2-2 \cos \sigma_{0}, & \text { solenoidal focusing, } \\ \sqrt{2-2 \cos \sigma_{0}}, & \text { quadrupole focusing. }\end{cases}
$$

To analyze the envelope stability of the fully space-charge depressed beam in solenoidal and quadrupole focusing channels we examine the change in the envelope coordinate vector $\mathbf{R}(s)=$ $\left(R_{+}(s), R_{+}^{\prime}(s), \zeta(s) R_{-}(s), \zeta(s) R_{-}^{\prime}(s)\right)$ from the mid-drift at $s=0$ to the next mid-drift at $s=L$. Here, $\zeta(s)=1$ when the next lens to be traversed is focusing, and $\zeta(s)=$ -1 when the next lens is defocusing. For solenoidal 
focusing $\zeta(s)=1$, and for alternating gradient quadrupole focusing $\zeta(s)=-1$ for $0 \leq s \leq L / 2$ and $\zeta(s)=1$ for $L / 2<s \leq L$. The effect of $\zeta(s)$ is to map the matched beam envelope $R_{-}$in the second drift region of the quadrupole $(L / 2<s \leq L)$ into the first drift region $(0 \leq$ $s<L / 2$ ) [see Fig. 24(c)]. This mapping expedites the use of symmetry in the matched envelope to simplify calculations for the FODO lattice.

To analyze the first-order perturbations in the coordinate vector $\mathbf{R}(s)$ we compute the Jacobian matrix $\mathbf{M}(0, L)$ where $\mathbf{M}\left(s_{1} \mid s_{2}\right)=\partial \mathbf{R}\left(s_{2}\right) / \partial \mathbf{R}\left(s_{1}\right)$ and derivatives are evaluated for a matched beam envelope. The Jacobian matrix $\mathbf{M}(0 \mid L)$ is identical to the symplectic transfer map in Eq. (46). Analogously to the treatment in Sec. II F, the stability of the envelope to linear perturbations about the matched envelope solution corresponds to $\mathbf{M}(0 \mid L)$ having all eigenvalues on the complex unit circle. To characterize mode properties, eigenvalues will be calculated in terms of $\sigma_{0}$.

In calculating $\mathbf{M}(0 \mid L)$, we henceforth denote $\mathcal{F}(s \pm$ $0) \equiv \lim _{\delta \rightarrow \pm 0} \mathcal{F}(s+\delta)$ to represent the discontinuous action of the thin lenses on the beam envelope functions. To exploit lattice symmetries, we split the interval $(0, L)$ into three parts $\left(0, \frac{L}{2}-0\right),\left(\frac{L}{2}-0, \frac{L}{2}+0\right)$, and $\left(\frac{L}{2}+0, L\right)$, and calculate $\mathbf{M}(0, L)$ as

$$
\mathbf{M}(0 \mid L)=\mathbf{M}\left(\frac{L}{2}+0 \mid L\right) \mathbf{M}\left(\frac{L}{2}-0 \mid \frac{L}{2}+0\right) \mathbf{M}\left(0 \mid \frac{L}{2}-0\right) .
$$

By symmetry, $\mathbf{M}\left(\frac{L}{2}+0 \mid L\right)=\mathbf{M}\left(0 \mid-\frac{L}{2}+0\right)^{-1}$ and so this can be expressed as

$$
\mathbf{M}(0 \mid L)=\mathbf{M}_{f}\left(-\frac{L}{2}+0\right)^{-1} \mathbf{M}_{s} \mathbf{M}_{f}\left(\frac{L}{2}-0\right),
$$

$$
\begin{aligned}
\ln \frac{R_{+}(s)}{R_{+}(0)} & =-R_{+}^{\prime 2}(0)+\left\{\operatorname{erfi}^{(-1)}\left[\operatorname{erfi}\left(R_{+}^{\prime}(0)\right)+e^{R_{+}^{\prime 2}(0)} \frac{s}{\sqrt{\pi} R_{+}(0)}\right]\right\}^{2}, \\
R_{-}(s) & =R_{-}(0)+R_{-}^{\prime}(0) s, \\
R_{+}^{\prime 2}(s)-\ln R_{+}(s) & =\text { const. }
\end{aligned}
$$$$
\text { (0) }
$$

Here, $R_{ \pm}(0)$ and $R_{ \pm}^{\prime}(0)$ are the values of $R_{ \pm}(s)$ and $R_{ \pm}^{\prime}(s)$ at $s=0$. Equations (H6a) and (H6b) for $R_{ \pm}(s)$ satisfy Eqs. (H1a) and (H1b) in the free-drift region $|s|<L / 2$ where $\kappa_{x}(s)=0$. Employing Eq. (H6a) and $R_{+}^{\prime}(0)=0$, which follows from the matched beam symmetry $R_{+}(s)=R_{+}(-s)$, yields

$$
\mathbf{M}_{f}(s)=\left[\begin{array}{cccc}
\frac{R_{+}(s)-s R_{+}^{\prime}(s)}{R_{+}(0)} & 2 R_{+}(0) R_{+}^{\prime}(s) & 0 & 0 \\
-\frac{s}{2 R_{+}(0) R_{+}(s)} & \frac{R_{+}(0)}{R_{+}(s)} & 0 & 0 \\
0 & 0 & 1 & s \\
0 & 0 & 0 & 1
\end{array}\right]
$$

To complete the evaluation of $\mathbf{M}_{f}(L / 2-0)$, we find relations of the elements to $\sigma_{0}$ and $L$ by deriving equations connecting $R_{+}(L / 2-0) \equiv R_{+}(L / 2), R_{+}^{\prime}(L / 2-0)$, and $R_{+}(0)$ to these quantities for the matched beam envelope. By symmetry, for a periodic matched envelope

$$
\mathbf{M}_{s}=\left[\begin{array}{cccc}
1 & 0 & 0 & 0 \\
0 & 1 & -\frac{1}{f} & 0 \\
0 & 0 & -1 & 0 \\
\frac{1}{f} & 0 & 0 & -1
\end{array}\right]
$$

Using Eq. (H3), the elements of $\mathbf{M}_{s}$ can be expressed in terms of $\sigma_{0}$ and $L$.

To evaluate elements of $\mathbf{M}_{f}(s)$, the space-charge dominated free-drift solutions in Eqs. (18) and the Hamiltonian constraint in Eq. (D1) are employed. These equations, valid within the drift regions, can be expressed in the scaled $R_{ \pm}$coordinates as

where $\mathbf{M}_{s}=\mathbf{M}\left(\frac{L}{2}-0 \mid \frac{L}{2}+0\right)$ is the "singular Jacobian" $\mathbf{M}(0 \mid s)$ for $|s|<L / 2$ is the "free-drift Jacobian" associated with the half drift.

To evaluate $\mathbf{M}_{s}$, we consider the action of the thin lens according to Eq. (14). We obtain for the solenoidal chanand for the quadrupole channel keeping in mind that the $\zeta(s) R_{-}$changes sign from one free-drift region to the next,

$$
\begin{aligned}
& R_{+}^{\prime}\left(\frac{L}{2}-0\right)=-R_{+}^{\prime}\left(\frac{L}{2}+0\right), \\
& R_{-}^{\prime}\left(\frac{L}{2}-0\right)=-R_{-}^{\prime}\left(\frac{L}{2}+0\right) .
\end{aligned}
$$

For solenoids, Eqs. (H1a) and (H2) can be integrated once about $s=L / 2$ to show that

$$
\begin{aligned}
& R_{+}^{\prime}\left(\frac{L}{2}+0\right)=R_{+}^{\prime}\left(\frac{L}{2}-0\right)-\frac{1}{f} R_{+}\left(\frac{L}{2}\right), \\
& R_{-}^{\prime}\left(\frac{L}{2}+0\right)=R_{-}^{\prime}\left(\frac{L}{2}-0\right)-\frac{1}{f} R_{-}\left(\frac{L}{2}\right) .
\end{aligned}
$$

Combining these constraints with the matching conditions (H8), we get

$$
R_{+}^{\prime}\left(\frac{L}{2}-0\right)=\frac{1}{2 f} R_{+}\left(\frac{L}{2}\right), \quad R_{-}^{\prime}\left(\frac{L}{2}-0\right)=\frac{1}{2 f} R_{-}\left(\frac{L}{2}\right) .
$$

Similarly, using Eqs. (H1b) and (H2) for alternating gradient focusing and matched beam symmetries (H8), we obtain

$$
R_{+}^{\prime}\left(\frac{L}{2}-0\right)=\frac{1}{2 f} R_{-}\left(\frac{L}{2}\right), \quad R_{-}^{\prime}\left(\frac{L}{2}-0\right)=\frac{1}{2 f} R_{+}\left(\frac{L}{2}\right) .
$$


For quadrupole focusing, Eqs. (H9b) and (14) can be combined to yield an additional constraint

$$
R_{+}^{\prime}\left(\frac{L}{2}-0\right)=\frac{L}{8 f^{2}} R_{+}\left(\frac{L}{2}\right) .
$$

Both the solenoidal and quadrupole matching conditions in Eqs. (H9a) $-(\mathrm{H} 9 \mathrm{c})$ for $R_{+}$can be expressed as

$$
R_{+}^{\prime}\left(\frac{L}{2}-0\right)=\frac{\hat{k}}{L} R_{+}\left(\frac{L}{2}\right)
$$

where

$$
\hat{k}= \begin{cases}\frac{L}{2 f}=1-\cos \sigma_{0}, & \text { solenoidal focusing, } \\ \frac{L^{2}}{8 f^{2}}=\frac{1}{4}\left(1-\cos \sigma_{0}\right), & \text { quadrupole focusing. }\end{cases}
$$

Applying the constant, drift region envelope Hamiltonian in Eq. (H6c) between $s=0$ and $s=L / 2-0$ and taking $R_{+}^{\prime}(0)=0$ consistent with a matched beam equilibrium in the resulting expression leads to the constraint

$$
R_{+}\left(\frac{L}{2}\right)=R_{+}(0) e^{R_{+}^{\prime 2}(L / 2-0)} .
$$

Then evaluating the free-drift expansion formula (H6a) at $s=L / 2$ with $R_{+}^{\prime}(0)=0$ and using Eq. (H11) to simplify the result yields

$$
\frac{L}{R_{+}(0)}=2 \sqrt{\pi} \operatorname{erfi}\left(R_{+}^{\prime}\left(\frac{L}{2}-0\right)\right) \text {. }
$$

Equations (H10) $-(\mathrm{H} 12)$ produce a relation between $\sigma_{0}$ and $R_{+}^{\prime}\left(\frac{L}{2}-0\right)$ :

$$
\begin{aligned}
& \hat{k}=2 \sqrt{\pi} e^{-R_{+}^{\prime 2}(L / 2-0)} R_{+}^{\prime}\left(\frac{L}{2}-0\right) \operatorname{erfi}\left(R_{+}^{\prime}\left(\frac{L}{2}-0\right)\right) \text {. (H13) } \\
& \mathbf{M}_{+}(0 \mid L)=\left[\begin{array}{cc}
\frac{R_{+}\left(-\frac{L}{2}\right)+\frac{L}{2} R_{+}^{\prime}\left(-\frac{L}{2}\right)}{R_{+}(0)} & 2 R_{+}(0) R_{+}^{\prime}\left(-\frac{L}{2}\right) \\
\frac{\frac{L}{2}}{2 R_{+}(0) R_{+}\left(-\frac{L}{2}\right)} & \frac{R_{+}(0)}{R_{+}\left(-\frac{L}{2}\right)}
\end{array}\right]^{-1}\left[\begin{array}{cc}
1 & 0 \\
-\frac{1}{f} & 1
\end{array}\right]\left[\begin{array}{cc}
\frac{R_{+}\left(\frac{L}{2}\right)-\frac{L}{2} R_{+}^{\prime}\left(\frac{L}{2}\right)}{R_{+}(0)} & 2 R_{+}(0) R_{+}^{\prime}\left(\frac{L}{2}\right) \\
\frac{L}{2} & \frac{R_{+}(0)}{2 R_{+}(0) R_{+}\left(\frac{L}{2}\right)}
\end{array}\right] \\
& =\left[\begin{array}{cc}
\cos \sigma_{0}-4 R_{+}^{\prime 2}\left(\frac{L}{2}\right) \cos ^{2}\left(\frac{\sigma_{0}}{2}\right) & 2 \frac{R_{+}^{2}(0)}{f}\left[1-2 R_{+}^{\prime 2}\left(\frac{L}{2}\right)\right] \\
\frac{-f}{R_{+}^{2}(0)} \cos ^{2}\left(\frac{\sigma_{0}}{2}\right)\left[1-\cos \sigma_{0}+4 R_{+}^{\prime 2}\left(\frac{L}{2}\right) \cos ^{2}\left(\frac{\sigma_{0}}{2}\right)\right] & \cos \sigma_{0}-4 \cos ^{2}\left(\frac{\sigma_{0}}{2}\right) R_{+}^{\prime 2}\left(\frac{L}{2}\right)
\end{array}\right], \\
& \mathbf{M}_{-}(0 \mid L)=\left[\begin{array}{cc}
1 & -\frac{L}{2} \\
0 & 1
\end{array}\right]^{-1}\left[\begin{array}{cc}
1 & 0 \\
-\frac{1}{f} & 1
\end{array}\right]\left[\begin{array}{ll}
1 & \frac{L}{2} \\
0 & 1
\end{array}\right]=\left[\begin{array}{cc}
\cos \sigma_{0} & \frac{L}{2}\left(1+\cos \sigma_{0}\right) \\
-\frac{2}{L}\left(1-\cos \sigma_{0}\right) & \cos \sigma_{0}
\end{array}\right] \text {. }
\end{aligned}
$$

Equations (H10)-(H13) provide the needed constraints to relate the elements of $\mathbf{M}_{f}(L / 2-0)$ to $\sigma_{0}$ and $L$. Needed elements of $\mathbf{M}_{f}(-L / 2+0)$ can be calculated from these constraints using the matched beam symmetry requirements

$$
R_{+}\left(-\frac{L}{2}\right)=R_{+}\left(\frac{L}{2}\right), \quad R_{+}^{\prime}\left(-\frac{L}{2}+0\right)=-R_{+}^{\prime}\left(\frac{L}{2}-0\right) .
$$

Henceforth, to abbreviate formulas, we denote $R_{+}^{\prime}(L / 2-$ $0) \equiv R_{+}^{\prime}(L / 2)$ and $R_{+}^{\prime}(-L / 2+0) \equiv R_{+}^{\prime}(-L / 2)$.

For solenoidal focusing $R_{ \pm}$are uncoupled, and $\mathbf{M}(0 \mid L)$ is of block diagonal form with [see Eq. (50)]

$$
\mathbf{M}(0 \mid L)=\left[\begin{array}{cc}
\mathbf{M}_{+}(0 \mid L) & 0 \\
0 & \mathbf{M}_{-}(0 \mid L)
\end{array}\right]
$$

where $\mathbf{M}_{ \pm}(0 \mid L)$ are $2 \times 2$ symplectic matrices that can be independently analyzed for the stability of perturbations in $R_{+}$(breathing mode) and $R_{-}$(quadrupole mode). Mode phase advances $\left(\sigma_{ \pm}\right)$and growth factors $\left(\gamma_{ \pm}\right)$can be calculated from the eigenvalues $\lambda_{ \pm}$of $\mathbf{M}_{ \pm}(0 \mid L)$. Stable modes with $\lambda_{ \pm}$on the complex unit circle correspond to $(1 / 2)\left|\operatorname{Tr} \mathbf{M}_{ \pm}(0 \mid L)\right|<1$ [see Eq. (52)]. The matrices $\mathbf{M}_{ \pm}(0 \mid L)$ can be evaluated from Eqs. (H4) using Eqs. (H5a) and (H7). Resulting expressions are simplified

Eigenvalues $\lambda_{ \pm}$of the matrices $\mathbf{M}_{ \pm}(0 \mid L)$ are calculated from Eq. (H15) as

$$
\lambda_{+}=\cos \sigma_{0}-4 R_{+}^{\prime 2}\left(\frac{L}{2}\right) \cos ^{2}\left(\frac{\sigma_{0}}{2}\right) \pm 2 i \cos \left(\frac{\sigma_{0}}{2}\right) \sqrt{\left[1-2 R_{+}^{\prime 2}\left(\frac{L}{2}\right)\left[\sin ^{2}\left(\frac{\sigma_{0}}{2}\right)+2 R_{+}^{\prime 2}\left(\frac{L}{2}\right) \cos ^{2}\left(\frac{\sigma_{0}}{2}\right)\right]\right.}, \quad \lambda_{-}=\cos \sigma_{0} \pm i \sin \sigma_{0} .
$$

From these eigenvalues, we calculate mode phase advances

$$
\sigma_{+}=\arg \lambda_{+} \quad \text { with }+ \text { sign in Eq. (H16), } \quad \sigma_{-}=\sigma_{0},
$$

and growth factors

$$
\gamma_{+}=\left\{\begin{array}{ll}
1, & \text { in stable regions, } \\
\sqrt{2\left[\cos \sigma_{0}-4 R_{+}^{\prime 2}\left(\frac{L}{2}\right) \cos ^{2}\left(\frac{\sigma_{0}}{2}\right)\right]^{2}-1,} & \text { in unstable regions, }
\end{array} \quad \gamma_{-}=1 .\right.
$$

These solutions for $\sigma_{ \pm}$and $\gamma_{ \pm}$are plotted in Fig. 25 as a function of $\sigma_{0}$ using the constraint equation (H13) to numerically eliminate $R_{+}^{\prime}(L / 2)$ in terms of $\sigma_{0}$. Continuous focusing results $\sigma_{+}=\sqrt{2} \sigma_{0}$ and $\sigma_{-}=\sigma_{0}$ [see Eq. (64) with $\sigma=0$ ] are superimposed on the phase advance plot for branch comparison. The extent of the band of instability $\left(\gamma_{+} \neq 1\right)$ in $\sigma_{0}$ can be calculated from $\gamma_{+}$directly or by examining where the stability condition [see Eq. (52)] 

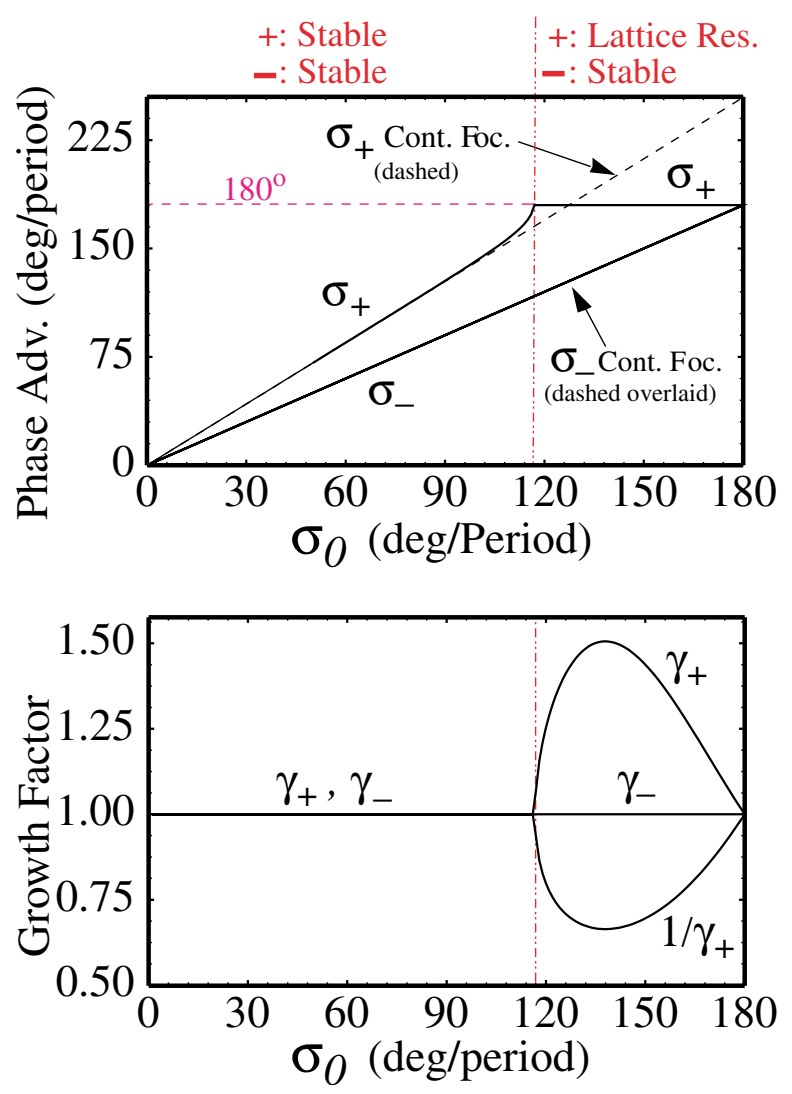

FIG. 25. (Color) Phase advance $\left(\sigma_{ \pm}\right)$and growth factors $\left(\gamma_{ \pm}\right)$ for the breathing and quadrupole modes versus $\sigma_{0}$ for a thinlens solenoidal focusing channel and a fully depressed beam with $\sigma=0$. Continuous focusing model predictions for $\sigma_{ \pm}$are indicated with dashed lines.

$$
\frac{1}{2}\left|\operatorname{Tr} \mathbf{M}_{+}(0 \mid L)\right|=\cos \sigma_{0}-4 R_{+}^{\prime 2}\left(\frac{L}{2}\right) \cos ^{2}\left(\frac{\sigma_{0}}{2}\right)<1
$$

is violated. This occurs when $R_{+}^{\prime 2}(L / 2)>1 / 2$ for $\sigma_{0}<\pi$. Using Eqs. (H10)-(H13) we conclude that the instability band for solenoidal focusing is located on the interval

$$
\sigma_{0} \in\left[\arccos \left(1-\sqrt{\frac{2 \pi}{e}} \operatorname{erfi}\left(\frac{1}{\sqrt{2}}\right)\right), \pi\right] \approx\left[116.715^{\circ}, 180^{\circ}\right]
$$

Here, we have denoted the boundary points of the interval to be unstable based on an analysis of the matrices $\mathbf{M}_{ \pm}(0 \mid L)$ which shows that there is linear growth in $R_{+}$when $R_{+}^{\prime 2}(L / 2)=1 / 2$, and linear growth in $R_{-}$when $\sigma_{0}=\pi$.

The stability of quadruple focusing can be investigated analogously to the case of solenoidal focusing except that we must work with the full $4 \times 4$ Jacobian matrix $\mathbf{M}(0 \mid L)$ because $R_{ \pm}$are coupled in this case. After multiplying out the matrices in Eq. (H4) and employing Eqs. (H9c) and (H14), we obtain

$$
\mathbf{M}(0 \mid L)=\left[\begin{array}{cccc}
1-\frac{L^{2}}{8 f^{2}} & \frac{L}{2 f^{2}} R_{+}^{2}(0) & -\frac{L}{4 f^{3}} R_{+}(0) R_{+}\left(\frac{L}{2}\right) & -\frac{L^{2}}{8 f^{2}} R_{+}(0) R_{+}\left(\frac{L}{2}\right) \\
\frac{L\left(L^{2} / f^{2}-16\right)}{32 R_{+}(0)} & 1-\frac{L^{2}}{8 f^{2}} & \frac{\left(L^{2} / f^{2}-16\right) R_{+}\left(\frac{L}{2}\right)}{16 f R_{+}(0)} & \frac{L\left(L^{2} / f^{2}-16\right) R_{+}\left(\frac{L}{2}\right)}{32 f R_{+}(0)} \\
\frac{-L\left(L^{2} / f^{2}-16\right) R_{+}\left(\frac{L}{2}\right)}{32 f R_{+}(0)} & \frac{L^{2}}{8 f^{3}} R_{+}(0) R_{+}\left(\frac{L}{2}\right) & -1 & -L \\
\frac{-\left(L^{2} / f^{2}-16\right) R_{+}\left(\frac{L}{2}\right)}{16 f R_{+}(0)} & \frac{L}{4 f^{3}} R_{+}(0) R_{+}\left(\frac{L}{2}\right) & 0 & -1
\end{array}\right] .
$$

The four eigenvalues $\lambda$ of $\mathbf{M}(0 \mid L)$ are calculated from Eq. (H20) and then simplified using the constraints in Eqs. (H10)-(H13). We obtain

$$
\lambda=w-\frac{1}{4} \sin ^{2}\left(\frac{\sigma_{0}}{2}\right) \pm i \sqrt{\frac{1}{2} w \sin ^{2}\left(\frac{\sigma_{0}}{2}\right)+\frac{1}{2}\left[1-\frac{1}{4} \sin ^{2}\left(\frac{\sigma_{0}}{2}\right)\right]\left[\sin ^{2}\left(\frac{\sigma_{0}}{2}\right)+16 R_{+}^{\prime 2}\left(\frac{L}{2}\right)\right]},
$$

where 


$$
w= \pm \sqrt{\left[1-\frac{1}{4} \sin ^{2}\left(\frac{\sigma_{0}}{2}\right)\right]\left[1-\frac{1}{4} \sin ^{2}\left(\frac{\sigma_{0}}{2}\right)-8 R_{+}^{\prime 2}\left(\frac{L}{2}\right)\right]} .
$$

These eigenvalues can be employed to calculate phase advances $\left(\sigma_{B}\right.$ and $\left.\sigma_{Q}\right)$ and growth factors $\left(\gamma_{B}\right.$ and $\left.\gamma_{Q}\right)$ of the breathing and quadrupole modes as $\sigma_{B, Q}=2 \arg \lambda$ and $\gamma_{B, Q}=\left|\lambda^{2}\right|$. Here, the factors of 2 stem from $\mathbf{M}(0 \mid L)$ being a half-period advance in a symmetric FODO lattice with period $2 L$. The physical branches of $\lambda$ selected according to the prescriptions given in Sec. II F correspond to Taylor series $\sigma_{B}=\sqrt{2} \sigma_{0}\left[1+(1 / 768) \sigma_{0}^{4}+\right.$ $\left.(211 / 967680) \sigma_{0}^{6}+\cdots\right]$ and $\sigma_{Q}=\sigma_{0}\left[1+(1 / 96) \sigma_{0}^{2}+\right.$ $\left.(203 / 92160) \sigma_{0}^{4}+\cdots\right]$. These solutions are plotted in Fig. 26 as a function of $\sigma_{0}$ using the constraint equation (H13) to numerically eliminate $R_{+}^{\prime}(L / 2)$ in terms of $\sigma_{0}$. Using Eqs. (H13) and (H21) we find numerically that the instability band is located on the interval

$$
\sigma_{0} \in\left(121.055^{\circ}, 180^{\circ}\right)
$$

[1] I. Kapchinskij and V. Vladimirskij, in Proceedings of the International Conference on High Energy Accelerators and Instrumentation (CERN Scientific Information Service, Geneva, 1959), p. 274.

[2] M. Reiser, Theory and Design of Charged Particle Beams (John Wiley \& Sons, Inc., New York, 1994).

[3] R. C. Davidson and H. Qin, Physics of Intense Charged Particle Beams in High Energy Accelerators (World Scientific, Singapore, 2001).

[4] I. Hofmann, L. J. Laslett, L. Smith, and I. Haber, Part. Accel. 13, 145 (1983).

[5] P. M. Lapostolle, IEEE Trans. Nucl. Sci. 18, 1101 (1971).

[6] F. J. Sacherer, IEEE Trans. Nucl. Sci. 18, 1105 (1971).

[7] J. Struckmeier and M. Reiser, Part. Accel. 14, 227 (1984).

[8] R. L. Gluckstern, Phys. Rev. Lett. 73, 1247 (1994).

[9] Some authors prefer to put factors of $\pi$ in the emittance definitions in Eq. (5) (i.e., $\varepsilon_{j} \rightarrow \varepsilon_{j} / \pi$ ), so that the emittances $\varepsilon_{x}$ and $\varepsilon_{y}$ are the $x-x^{\prime}$ and $y-y^{\prime}$ phase-space areas of a KV distribution.

[10] S. M. Lund and R. C. Davidson, Phys. Plasmas 5, 3028 (1998).

[11] T. Wangler, K. Crandall, R. Mills, and M. Reiser, IEEE Trans. Nucl. Sci. 32, 2196 (1985).

[12] No proof exists that such a Vlasov self-consistent distribution can or cannot be constructed. A self-consistent distribution of this form would not necessarily imply an equilibrium. For example, in a periodic lattice, $n(\rho)$ could change in form from period to period. Some authors (see, for example, Ref. [48]) have assumed constructs where $n(\rho)$ maintains self-similar form in a periodic lattice. However, such assumptions are implausible under the Vlasov evolution.

[13] S. M. Lund, J. J. Barnard, and E. P. Lee, in Proceedings of the $X X$ International Linac Conference, Monterey, CA, 2000 (SLAC, Stanford, CA, 2000), p. 290 and MOE11.
[14] S. M. Lund, J. J. Barnard, and J. Miller, in Proceedings of the 1995 Particle Accelerator Conference, Dallas, TX (IEEE, Piscataway, NJ, 1995), p. 3278.

[15] This result can be proved for small-amplitude mismatch excursions about a matched beam using results developed later. No fully nonlinear proof is known for large-amplitude mismatches, although numerical results support the conjecture.

[16] S. Humphries, Charged Particle Beams (John Wiley \& Sons, Inc., New York, 1990).

[17] J. S. O'Connell, J. Appl. Phys. 70, 7156 (1991).

[18] E. D. Courant and H.S. Snyder, Ann. Phys. (N.Y.) 3, 1 (1958).

[19] H. Wiedemann, Particle Accelerators Physics: Basic Principles and Linear Beam Dynamics (SpringerVerlag, New York, 1993).

[20] R. Pakter and F. B. Rizzato, Phys. Rev. Lett. 87, 044801 (2001).

[21] R. Pakter and F. B. Rizzato, Phys. Rev. E 65, 056503 (2002).

[22] Caution must be used when interpreting Refs. [20,21] because the authors employ $\sigma_{0}$ as a focusing parameter which does not correspond to the undepressed particle phase advance.

[23] E. Lee and R. J. Briggs, Lawrence Berkeley National Laboratory Technical Report No. LBNL-40774, UC419, 1997.

[24] E. P. Lee, T. J. Fessenden, and L. J. Laslett, IEEE Trans. Nucl. Sci. 26, 2489 (1985).

[25] E. P. Lee, Phys. Plasmas 9, 4301 (2002).

[26] C. Chen and R. C. Davidson, Phys. Rev. Lett. 72, 2195 (1994).

[27] Q. Qian and R. C. Davidson, Phys. Rev. E 53, 5349 (1996).

[28] A. Dragt, in Physics of High Energy Particle Accelerators, edited by R. A. Carrigan, F. R. Hudson, and M. Month, AIP Conf. Proc. No. 87 (AIP, New York, 1982), p. 147.

[29] J. J. Barnard, S. M. Lund, and M. de Hoon, Lawrence Berkeley National Laboratory Technical Report No. LBNL-49286, 2001, United States Particle Accelerator School, Course Notes, Boulder, CO.

[30] V. P. Karpenko, P. A. Seidl, R. M. Franks, and S. M. Lund, in Proceedings of the 2001 Particle Accelerator Conference (IEEE, Piscataway, NJ, 2001), p. 1447.

[31] P. A. Seidl, D. Baca, F. M. Bieniosek, A. Faltens, S. M. Lund, A.W. Molvik, L. R. Prost, D. B. Shuman, and W. L. Waldron, Laser Part. Beams 20, 435 (2002).

[32] H. Wiedemann, Particle Accelerators Physics II: Nonlinear and Higher-Order Beam Dynamics (Springer-Verlag, New York, 1995).

[33] F. J. Sacherer, Ph.D. thesis, University of California, 1968.

[34] E. P. Lee and R. K. Cooper, Part. Accel. 7, 83 (1976).

[35] R. B. Miller, An Introduction to the Physics of Intense Charged Particle Beams (Plenum Press, New York, 1982).

[36] A. J. Dragt, F. Neri, and G. Rangarajan, Phys. Rev. A 45, 2572 (1992).

[37] R. A. Kishek, J. J. Barnard, and D. P. Grote, in Proceedings of the 1999 Particle Accelerator 
Conference, New York City (IEEE, Piscataway, NJ, 1999), p. 1761.

[38] J. J. Barnard and B. Losic, in Proceedings of the $X X$ International Linac Conference, Monterey, CA, 2000 (Ref. [13]), p. 293 and MOE12.

[39] The alternating gradient case with $P_{\theta} \neq 0$ can occur in lattices with skew quadrupoles or where the principal axes of the elliptical beam are not initially aligned with the quadrupole axes.

[40] C. Chen, R. Pakter, and R. C. Davidson, Phys. Rev. Lett. 79, 225 (1997).

[41] R. Ryne, acc-phys/9502001.

[42] For example, the TRACE 3D code, K. R. Crandall and D. P. Rusthoi, TRACE 3-D Documentation, Los Alamos National Laboratory Report No. LA-UR-97-886, 1997.

[43] An alternative trial matching condition can be constructed by taking $Q=0$ and $\varepsilon_{j}=0$ and numerically or analytically integrating the (linear) envelope equa- tions (4) subject to the periodicity requirement (7) [41]. This determines the solution to an overall scale factor that can be set using continuous focusing approximations (see Sec. III) or other low-order force balances (see Ref. [25]).

[44] C. K. Allen and M. Reiser, Nucl. Instrum. Methods Phys. Res., Sect. A 384, 322 (1997).

[45] D. P. Grote, A. Friedman, I. Haber, W. Fawley, and J.-L. Vay, Nucl. Instrum. Methods Phys. Res., Sect. A 415, 428 (1998).

[46] D. A. Edwards and L. C. Teng, IEEE Trans. Nucl. Sci. 20, 885 (1973).

[47] B. Bukh and S. M. Lund, in Proceedings of the 2003 Particle Accelerator Conference, Portland, OR (IEEE, Piscataway, NJ, 2003), p. WPPG019.

[48] Q. Qian, R. C. Davidson, and C. Chen, Phys. Rev. E 51, 5216 (1995). 